Portland State University

PDXScholar

Spring 5-1-2019

\title{
Fatigue Characteristics of New ECO Series Aluminum 7175 Alloy
}

Chinh Q.L. Vu

Portland State University

Follow this and additional works at: https://pdxscholar.library.pdx.edu/open_access_etds

Part of the Mechanical Engineering Commons

Let us know how access to this document benefits you.

\section{Recommended Citation}

Vu, Chinh Q.L., "Fatigue Characteristics of New ECO Series Aluminum 7175 Alloy" (2019). Dissertations and Theses. Paper 4985.

https://doi.org/10.15760/etd.6861

This Dissertation is brought to you for free and open access. It has been accepted for inclusion in Dissertations and Theses by an authorized administrator of PDXScholar. Please contact us if we can make this document more accessible: pdxscholar@pdx.edu. 
Fatigue Characteristics of New ECO Series Aluminum 7175 Alloy

by

Chinh Q.L. Vu

A dissertation submitted in partial fulfillment of the requirements for the degree of

\author{
Doctor of Philosophy \\ in \\ Mechanical Engineering \\ Dissertation Committee: \\ Sung Yi, Chair \\ Chien Wern \\ Faryar Etesami \\ Franz Rad
}

Portland State University

2019 
(C) 2019 Chinh Q.L. Vu 


\begin{abstract}
In this dissertation, the fatigue characteristics of three newly developed experimental compositions for aluminum 7175, with improved mechanical strength, that uses magnesium-calcium alloy instead of pure magnesium are studied.

Specimens of each variant were fabricated and subjected to fatigue life testing, fatigue life data analysis, and observation of their fracture characteristics through optical microscopy and scanning electron microscopy (SEM), and metallography to study their grains and surface characteristics.

Fatigue life testing shows all three variants have a fatigue strength that is approaching approximately $200 \mathrm{MPa}$. ECO7175v3 is shown to have the highest fatigue strength of approximately $220 \mathrm{MPa}$ at $5 \times 10^{7}$ cycles, approximately $40 \%$ of its tensile strength of $550 \mathrm{MPa}$. This is shown by its considerably higher fatigue strength coefficient determined by Basquin's equation compared to the other two variants. ECO7175v1 is shown to generally have large scatter in its fatigue life at higher stress levels $(65 \%$ or higher of their tensile strength) with coefficient of variations typically twice or more to those of ECO7175v2 and ECO7175v3.

The results of the SEM analysis show that irrespective of the stress levels, ECO7175v1 and ECO7175v3 all have crack initiation points at the surface with no inclusions to act as stress concentrators. The lack of inclusions is supported by the reliability analysis which shows the hazard rates for all variants remains relatively constant most of the time before increasing towards the end. These trends for all variants indi-
\end{abstract}


cate failures are due to wear-outs instead of defects, which were not seen. Reliability analysis also shows that at any given fatigue life cycle and stress level, ECO7175v3 has a lower probability of failure when compared to ECO7175v1 and ECO7175v2. On the other hand, at any given fatigue life cycle and stress level, ECO7175v1 is shown to have a higher probability of failure when compared to ECO7175v2 and ECO7175v3. 


\section{Acknowledgments}

I want to thank my advisor, Dr. Sung Yi, for sincerely advising me and guiding me throughout my entire graduate studies at Portland State University, to Dr. Chien Wern and Dr. Faryar Etesami for all the valuable discussions, sharing of experiences, and allowing me to learn and utilize various mechanical equipment as well as supporting me during my undergraduate studies, Dr. Tae-Kyu Lee, and Dr. Graham Tewksbury for helping me with my various questions, to Dr. Ji-Woon Lee and Je-Sik Shin from the Korea Institute of Industrial Technology (KITECH) for their support and the coffee breaks as well as Dr. Shae Kwang Kim and Dr. Bong-Hwan Kim who helped me with this research. In addition, I want to give my gratitude to Tricia Hutchins, Crystal Snelson, and Sarah Santucci for always being there to cheerfully supporting me, keeping me on top of things, and talking to me about real life outside of the university, and Mike Chuning for always requesting me for the machine shop. I also want to give my thanks to my fellow graduate students and others in no particular order: Recently graduated Dr. Kyung-Eun Min, Ryan Smith, Thomas Langston, Tom Bennet, Zdenek Zumr, Dr. Jun Jiao, Dr. Lemmy Meekisho, Dr. Zareh Hormoz, Dr. David Turcic, Dr. Gerald Recktenwald, Greg Baty, Dr. Matthew Melius, Hawwa Kadum, Dr. Yongkang Chen, Nguyen Le, Valeri Yunusa, Dr. Manisha Dixit, and Aditya Thakare. Finally, I want to thanks my parents for raising me, guiding me through life, and supporting me all the way on this long endeavor, because without them, I wouldn't be here. 


\section{Table of Contents}

Abstract $\quad$ i

Acknowledgements $\quad$ iii

List of Tables $\quad$ vi

List of Figures viii

$\begin{array}{lll}\text { Chapter } 1 & \text { Introduction } & 1\end{array}$

1.1 Aluminum Alloys . . . . . . . . . . . . . . . . . . . . 1

$\begin{array}{lll}\text { Chapter } 2 & \text { Background Theory } & 7\end{array}$

2.1 Fatigue of Materials . . . . . . . . . . . . . . . . . . 7

2.2 Fatigue Analysis Approach . . . . . . . . . . . . . . . . . . . 12

2.3 Reliability Analysis . . . . . . . . . . . . . . . . . 15

2.4 Estimating Reliability . . . . . . . . . . . . . . . . . . 21

$\begin{array}{lll}\text { Chapter } 3 & \text { Experimental Setup } & 28\end{array}$

$\begin{array}{lll}\text { Chapter } 4 & \text { ECO7175v1-T74 } & 32\end{array}$

4.1 Basquin's Equation . . . . . . . . . . . . . . . . 36 
4.2 Fracture Analysis . . . . . . . . . . . . . . . . . . 37

$\begin{array}{lll}\text { Chapter } 5 & \text { ECO7175v3-T74 } & 44\end{array}$

5.1 Basquin's Equation . . . . . . . . . . . . . . . . 47

5.2 Fracture Analysis . . . . . . . . . . . . . . . . . . . . . . . . . . . . . 48

5.3 Metallography ....................... 59

$\begin{array}{llr}\text { Chapter } 6 & \text { ECO7175v2-T74 } & 67\end{array}$

6.1 Fracture Analysis . . . . . . . . . . . . . . . . . . 71

6.2 Metallography..................... 75

6.3 Basquin's Equation . . . . . . . . . . . . . . . . . . 79

$\begin{array}{lll}\text { Chapter } 7 & \text { Discussions } & 80\end{array}$

7.1 General Analysis . . . . . . . . . . . . . . . . . . . . . 80

7.2 Fatigue and Fracture Analysis . . . . . . . . . . . . . . . . 82

7.3 Metallography.................... 83

7.4 Reliability Analysis . . . . . . . . . . . . . . . . . 84

7.5 Basquin's Equation . . . . . . . . . . . . . . . . . 101

$\begin{array}{lll}\text { Chapter } 8 & \text { Conclusion } & 103\end{array}$

$\begin{array}{ll}\text { Bibliography } & 105\end{array}$ 


\section{List of Tables}

1.1 Manufactured composition of aluminum ECO7175 and standard reference aluminum 7175. Bold elements were intentionally added. Non-bold elements existed as natural impurities in commercial pure aluminum ingots. Compositions in wt\%. .................... 5

4.1 Allocation of Specimens for ECO7175v1-T74. . . . . . . . . . . . 33

4.2 Manufactured Composition of Aluminum ECO7175v1-T74. . . . . . . . . 33

4.3 Tensile Properties of Aluminum 7175. . . . . . . . . . . . . . . . . . 34

4.4 Statistical Analysis of Fatigue Testing for ECO7175v1-T74. . . . . . . . . 35

5.1 Allocation of Specimens for ECO7175v3-T74. . . . . . . . . . . . 44

5.2 Manufactured Composition of Aluminum ECO7175v3-T74. . . . . . . . 45

5.3 Statistical Analysis of Fatigue Testing for ECO7175v3-T74. . . . . . . . . 46

5.4 EDX (Energy-dispersive X-ray spectroscopy) Spot Analysis for ECO7175v3-

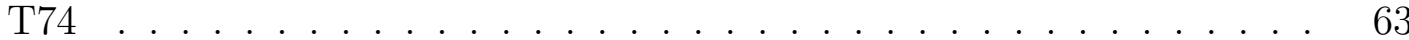

6.1 Allocation of Specimens for ECO7175v2-T74. . . . . . . . . . . . 67

6.2 Manufactured Composition of Aluminum ECO7175v2-T74. . . . . . . . 68

6.3 Statistical Analysis of Fatigue Testing for ECO7175v2-T74. . . . . . . . . 68 
7.1 Manufactured composition of aluminum ECO7175 and standard reference aluminum 7175. Bold elements were intentionally added. Non-bold elements existed as natural impurities in commercial pure aluminum ingots. Compositions in wt\%. . . . . . . . . . . . . . . . . 81

7.2 ECO7175's Striations' Width. . . . . . . . . . . . . . 83

7.3 Statistical results of Weibull fitting of ECO7175 at $65 \%$ UTS. . . . . . . 90

7.4 Statistical results of log-rank test for ECO7175. . . . . . . . . . . . . 100

7.5 Basquin's Constants of ECO7175. . . . . . . . . . . . . . . 101 


\section{List of Figures}

\section{Chapter 2}

2.11842 Versailles rail accident in France [13]. . . . . . . . . . . . . . . . 9

2.21998 Eschede derailment in Germany [14]. . . . . . . . . . . . . . . . . 10

2.3 Fatigue Stress Parameters. . . . . . . . . . . . . . . . . . . . . . . 11

2.4 An example S-N curve with a material's endurance limit, $\sigma_{e}$, shown. . . . 12

2.5 Probability Density Function $(\mathrm{PDF}) \ldots \ldots \ldots$

2.6 Cumulative density function (CDF) and its complement. . . . . . . . . 18

2.7 Failure probability (CDF) and its complement, reliability. . . . . . . . 19

2.8 Bathtub curve of the hazard rate. . . . . . . . . . . . . . . . . 20

2.9 Weibull distribution for the hazard rate and its parameters. $\beta<1$ indicates early failures, $\beta=1$ indicates random failures, and $\beta>1$ indicates wear-out failures. . . . . . . . . . . . . . . . . . 25

2.10 Weibull distribution for the cumulative hazard function and its parameters. Similarly to the hazard rate, $\beta<1$ indicates early failures, $\beta=1$ indicates random failures, and $\beta>1$ indicates wear-out failures. . . . . . 26

2.11 Weibull distribution for reliability and its parameters. . . . . . . . .

\section{Chapter 3}


3.1 Fatigue coupon dimensions. Units are given in millimeter. . . . . . . . . 28

3.2 Model RFB200 fatigue tester by Fatigue Dynamics, Inc. . . . . . . . . . 29

\section{Chapter 4}

4.1 Heat treatment process for aluminum T74 temper. . . . . . . . . . . 34

4.2 S-N data plot of aluminum ECO7175v1-T74. . . . . . . . . . . . 35

4.3 Fatigue data for ECO7175v1-T74 [38] curve-fitted to Basquin's equation to determine the material's fatigue strength coefficient, $\sigma_{F}^{\prime}$, and fatigue strength exponent, b. . . . . . . . . . . . . . . . . 37

4.4 Specimens subjected to $86 \%$ UTS. $1 \mathrm{~mm}$ scale bar. Specimens at relatively higher stresses have more crack initiations points at the surface compared with specimens at relatively lower stresses. The crack initiation bands that propagate from these crack initiation points are also relatively shorter. 39

4.5 Specimens subjected to 44\% UTS. $1 \mathrm{~mm}$ scale bar. Specimens at lower stresses typically have fewer crack initiation points but typically have longer crack propagation bands that originate from these crack initiation

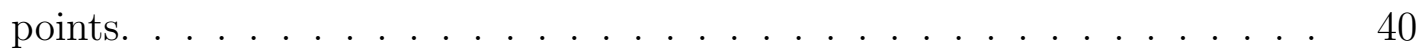

4.6 Left three specimens are subjected to $75 \%$ UTS. Right three specimens are subjected to $55 \%$ UTS. $1 \mathrm{~mm}$ scale bar. . . . . . . . . . . .

4.7 SEM photographs of specimens subjected to 44\% UTS (A); $55 \%$ UTS (B); $75 \%$ UTS $(\mathrm{C})$; and $86 \%$ UTS $(\mathrm{D}) \ldots \ldots \ldots \ldots$ 
4.8 SEM photographs of striations for ECO7175v1-T74 subjected to 75\% UTS. The striations, on average, have a width of approximately $0.481 \mu \mathrm{m}$. . . .

\section{Chapter 5}

5.1 S-N data plot of aluminum ECO7175v3-T74. At higher stress levels above $55 \%$ UTS, the scatter in the data are low. At stresses $55 \%$ UTS and lower, the scatter in the data are high. . . . . . . . . . . .

5.2 S-N data plot of aluminum ECO7175v3-T74 vs ECO7175v1-T74 [38]. Compared to ECO7175v1-T74, the scatter for ECO7175v3-T74 is generally lower. . . . . . . . . . . . . . . . . . . .

5.3 Fatigue data for ECO7175v3-T74 curve-fitted to Basquin's equation to determine the material's fatigue strength coefficient, $\sigma_{F}^{\prime}$, and fatigue strength exponent, $b \ldots \ldots \ldots \ldots \ldots \ldots$

5.4 Specimens subjected to 88\% UTS (left) and 77\% UTS (right) has fracture features that are well defined with long beach marks, clear crack initiation sites, and has failure modes that are predominately ductile near the surface at fracture initiation points while brittle towards the center closer to the final fracture rupture points. . . . . . . . . . . . . . 
5.5 Specimens subjected to $67 \%$ UTS (top), $57 \%$ UTS (center), $47 \%$ UTS (bottom), and below (not shown) has fracture features that are not well defined, have failure modes that are predominately ductile through the majority of the fracture surface, and are characteristically shiny. . . . . . 51

5.6 High power SEM images of ECO7175v3-T74 at various crack initiation points with no clear indication of inclusions to act as the stress concentrators. 52

5.7 The top figure shows a closeup near ECO7175v3-T74's crack initiation point. The bottom figure shows ECO7175v3-T74's fracture surfaces at other parts of the surface away from the crack initiation points. At the initiation points, the fracture is very sharp and looks like facets of gems, indicating it is a transgranular fracture where fracture cuts through the grains. At other areas away from the initiation points, the fractures are intergranular, meaning the fractures travels through the grain boundaries. $\quad 53$

5.8 Left three are ECO7175v3-T74 specimens subjected to $67 \%$ UTS (A), $57 \%$ UTS (B), and 47\% UTS (C). Right three are ECO7175v1-T74 specimens subjected to similar stresses at $66 \%$ UTS (D), 55\% UTS (E), and $44 \%$ $\operatorname{UTS}(\mathrm{F}) \ldots \ldots \ldots \ldots \ldots$

5.9 SEM images of ECO7175v3-T74 showing different surface characteristics across the fracture surface. In the top and bottom image, cone and cups can be ne noticed. These indicates the fracture at these points are ductile

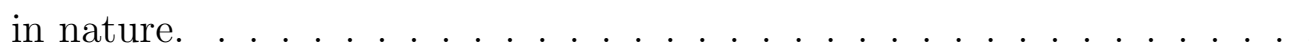


5.10 The top figure shows the a closeup of ECO7175v3-T74's surface. The bottom figure shows ECO7175v1-T74 at a similar magnification. Both surfaces have intergranular fracture characteristics but ECO7175v3-T74 has larger fractures. This is be caused by larger grain sizes in ECO7175v3. The larger grain sizes and the resulting fracture characteristics is what gives the ECO7175v3-T74's fracture surface a shiny look. . . . . . . . .

5.11 The top figure shows the a closeup of ECO7175v3-T74. The bottom figure shows ECO7175v1-T74 at a similar magnification. The larger grain sizes in ECO7175v3-T74 is what gives it its characteristic shiny fracture surface. 57

5.12 SEM photographs of striations for ECO7175v3-T74 subjected to 77\% UTS. The striations, on average, have a width of approximately $1.164 \mu \mathrm{m} . . . \quad 58$

5.13 Metallographs of ECO7175v3-T74 specimens showing the grains and grain boundaries. The average grain size is $78 \mu \mathrm{m}$. The grain sizes are considerably larger than the average grain size of $710 \mu \mathrm{m}$ for the other two variants. This is contributed by the very low chromium content, which is used to prevent recrystallization in aluminum-magnesium-zinc alloy [43]. . . . . 60

5.14 Metallographs of ECO7175v1-T74 specimens showing the grains and grain

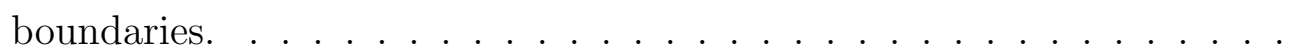


5.15 ECO7175v3-T74 polished surface under Nomarski exposed many irregular shaped bumps on the surface of the specimen. These bumps are recognized as precipitates as they are part of the matrix rather than surface contaminants. At 200x magnification. . . . . . . . . . . . . . 62

5.16 ECO7175v1-T74 polished surface under Nomarski. The precipitates are seemingly larger than precipitates on ECO7175v3-T74 specimens. At 200x magnification. . . . . . . . . . . . . . . .

5.17 Metallograph of highly polished surface of ECO7175v3-T74 under dark field imaging to highlight particles on the surface. At 100x magnification.

5.18 Metallograph of highly polished surface of ECO7175v1-T74 under dark field imaging to highlight particles on the surface. . . . . . . . .

5.19 Particle size distribution of ECO7175v3-T74 . . . . . . . . . . 65

5.20 Particle size distribution of ECO7175v1-T74. . . . . . . . . . . 66

5.21 Precipitates points of interests chosen for EDX (Energy-dispersive X-ray spectroscopy) analysis. . . . . . . . . . . . . . . . . 66

\section{Chapter 6}

6.1 S-N data plot of aluminum ECO7175v2-T74. . . . . . . . . . .

6.2 S-N data plot of aluminum ECO7175v1-T74 [38], ECO7175v2-T74, and

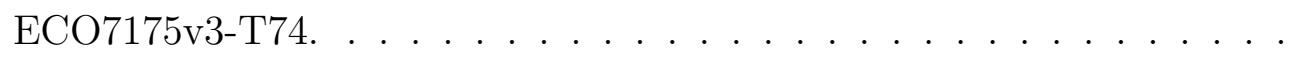


6.3 Specimens subjected to $75 \%$ UTS (right) have fracture characteristics that are well defined with long propagation bands, clear crack initiation sites, and have failure modes that are predominately ductile near the surface while brittle towards the center. Specimen subjected to $85 \%$ UTS (left) has similar characteristics to specimens subjected to $75 \%$ and higher on ECO7175v1-T74 with many crack initiation points around the rim and short propagation bands. . . . . . . . . . . . . . . . . 72

6.4 Left three are ECO7175v2-T74 specimens subjected to $65 \%$ UTS (A), $55 \%$ UTS (B), and 45\% UTS (C). Right three are ECO7175v3-T74 specimens subjected to similar stresses at $67 \%$ UTS (D), 57\% UTS (E), and $47 \%$

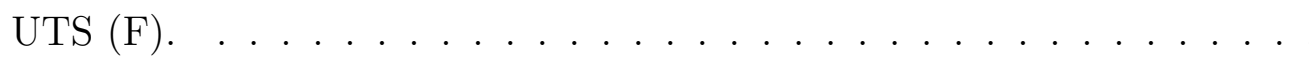

6.5 SEM photograph of striations for ECO7175v2-T74 subjected to 75\% UTS. The striations, on average, have a width of approximately $1.070 \mu \mathrm{m}$. The striation width is comparable to those of ECO7175v3-T74 but still considerably higher than those of ECO7175v1-T74. . . . . . . . . . . . 74

6.6 Metallograph of highly polished surface of ECO7175v2-T74 under dark field imaging to highlight the particles on the surface. At 100x magnification. Compared to the other two variants, the particles in ECO7175v2-T74 were incredibly hard to see as they are considerably smaller. . . . . . . . 76

6.7 Metallograph of highly polished surface of ECO7175v2-T74 under dark field imaging to highlight the particles on the surface. At 200x magnification. 77 
6.8 ECO7175v2-T74 specimens under Nomarski showed that compared to the other two variants, there were very little precipitates. The majority of what was noticed under the dark field imaging were in fact, pores. At 200x magnification. . . . . . . . . . . . . . . . 78

6.9 Particle size distribution of ECO7175v2-T74. . . . . . . . . . . . 78

6.10 Fatigue data for ECO7175v2-T74 curve-fitted to Basquin's equation to determine the material's fatigue strength coefficient, $\sigma_{F}^{\prime}$, and fatigue strength exponent, $b \ldots \ldots \ldots \ldots \ldots \ldots$

\section{Chapter 7}

7.1 Grains in the direction of TD for ECO7175v1 (top left) and ECO7175v2 (top right) are highly elongated while grains for ECO7175v3 (bottom left) are larger and equiaxed in all directions. . . . . . . . . . . . .

7.2 Kaplan-Meier estimator used to estimate the reliability of ECO7175-T74 at $85 \%$ UTS. The shade represents the $95 \%$ confidence interval. Although the confidence intervals are overlapping, the estimates show that in general, at any given fatigue life cycle and stress level, ECO7175v1 has lower reliability when compared to the other variants. . . . . . . . . .

7.3 Kaplan-Meier estimator used to estimate the reliability of ECO7175-T74

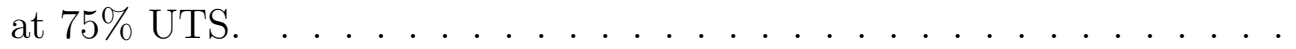


7.4 Kaplan-Meier estimator used to estimate the reliability of ECO7175-T74 at $65 \%$ UTS. ........................... 86

7.5 Kaplan-Meier estimator used to estimate the reliability of ECO7175-T74 at $55 \%$ UTS. . . . . . . . . . . . . . . . 87

7.6 Kaplan-Meier estimator used to estimate the reliability of ECO7175-T74

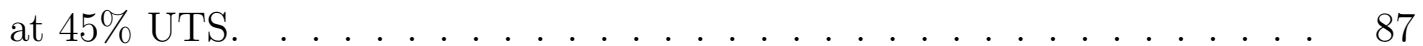

7.7 Kaplan-Meier estimator fitted to Weibull for ECO7175-T74 at 65\% UTS. 89

7.8 Cumulative hazard function of ECO7175 estimated with the Nelson-Aalen estimator at $75 \%$ UTS and then fitted to the Weibull distribution. . . . . 91

7.9 Cumulative hazard function of ECO7175 estimated with the Nelson-Aalen estimator at $55 \%$ UTS and then fitted to the Weibull distribution. . . . .

7.10 Calculated hazard rate for ECO7175 at 85\% UTS. . . . . . . . . . . . 93

7.11 Calculated hazard rate for ECO7175 at $75 \%$ UTS. . . . . . . . . . . . . 93

7.12 Calculated hazard rate for ECO7175 at $65 \%$ UTS. . . . . . . . . . . . 94

7.13 Calculated hazard rate for ECO7175 at 55\% UTS. . . . . . . . . . . . . 94

7.14 Calculated hazard rate for ECO7175 at $45 \%$ UTS. . . . . . . . . . 95

7.15 The left figure shows the calculated reliability and the estimated reliability using Kaplan-Meier. The results agree but the Kaplan-Meier is much simpler and efficient to implement. The right figure shows the estimated reliability and its Weibull fitting result. . . . . . . . . . . . . . . . 96

7.16 Probability of failure for ECO7175 at $85 \%$ UTS. . . . . . . . . . . . . . 97 
7.17 Probability of failure for ECO7175 at $75 \%$ UTS. . . . . . . . . . . . . 97

7.18 Probability of failure for ECO7175 at $65 \%$ UTS. . . . . . . . . . 98

7.19 Probability of failure for ECO7175 at 55\% UTS. . . . . . . . . . . 98

7.20 Probability of failure for ECO7175 at $45 \%$ UTS . . . . . . . . . . . . . . 99

7.21 Plot of Basquin's equations for all three variants of ECO7175. ECO7175v3 is shown to have a considerably higher fatigue strength coefficient compared to ECO7175v1-T74 and ECO7175v2-T74. . . . . . . . . . . 102 


\section{Chapter 1}

\section{Introduction}

\subsection{Aluminum Alloys}

Aluminum, or aluminium, is an abundant material that is relatively soft, ductile, malleable, and lightweight. Aluminum also has good strength to weight ratio, high corrosion resistance, and low thermal expansion. As a result, aluminum is widely used in various industries from automotive to the aerospace industry due to many of these attractive properties, especially as a structural material due to its high strength to weight ratio in applications where weight is of high importance. However, there is always a need for improved strength. Therefore, aluminum is typically alloyed with other elements to improve its strength. The increased strength is particularly important when taking fatigue resistance into consideration. Materials with higher strengths have higher fatigue resistance.

To improve upon its strength and other properties, aluminum is commonly alloyed with various elements such as copper and zinc. Aluminum alloys are available in series in which the standard naming scheme is designated by the International Alloy Designation System [1]. An alloy is given a four-digit number where the first digit indicates the major alloying element and also represent the series; the third and fourth digits are for identification of the specific alloy composition in the series; and the second digit, if it is not "0", indicates the variation of the alloy identified in the 
third and fourth digits. The aluminum 2000 series has copper as the primary alloying element, the 3000 series has manganese, the 4000 series has silicon, the 5000 series has magnesium, the 7000 series has zinc, and the 6000 series has combinations of elements such as magnesium and silicon. Finally, all other alloys not included in the standard 2000-7000 series, are placed in the 8000 series, such as aluminum alloys that used lithium as the primary alloying element.

In this dissertation, our interests are in aluminum 7175, from the 7000 series in which zinc is the primary alloying element. The use of zinc grants the 7000 series excellent combinations of strength and toughness. Thus, the 7000 series aluminum has the highest strength among the aluminum alloys [2]. As a result, 7000 series alumnum are often used in the aircraft industry and are slowly superseding the 2000 series due to their superior strength and corrosion resistance. Depending on the content of the other elements, their characteristics can be tweaked. For example, if copper is added or the content of copper is increased, they will have increased strength, at the expense of their corrosion resistance. On the other hand, if the content of magnesium is increased, their strength and corrosion resistance will both be further increased but their strength won't be increased to the same extent as increasing the copper content. In addition, the aluminum 7000 series can be further strengthened through traditional strengthening techniques such as solution and precipitation heat treatment. Among the 7000 series, aluminum 7075 has one of the highest strength. However, a variant of 7075 called 7175 was developed with the same exact composition but with fewer 
impurities due to an improved manufacturing process. The improved manufacturing process resulted in an aluminum 7175 with superior properties in strength and toughness compared to standard aluminum 7075 [3].

\section{ECO7175 Aluminum Alloy}

In this current dissertation, three different variants of ECO7175 aluminum are studied. ECO7175 aluminum alloy is a newly developed variant of the standard 7175 aluminum alloy by Korea Institute of Technology (KITECH). ECO in the variant name indicates that the material is more economical and environmentally friendly than the standard 7175 aluminum alloy series. The primary concept behind the new ECO aluminum alloys is the use of Eco-Mg, a magnesium-calcium alloy, instead of pure magnesium as the alloying element [4]. In the standard aluminum 7000 series, magnesium and zinc are the primary alloying elements. Magnesium is used as an alloying element because it significantly increased the strength of the alloy as well as the alloy's resistance to corrosion. However, magnesium is highly reactive and oxidative at high temperatures during processing and formation, which results in significant loss of magnesium through slag during the manufacturing process [5]. To compensate and reduce this loss, protective gases such as sulfur hexafluoride $\left(\mathrm{SF}_{6}\right)$ and fluxes are extensively used. These protective gases are both expensive and not environmentally friendly because sulfur hexafluoride is an extremely potent greenhouse gas. By alloying magnesium with calcium, the resulting magnesium alloy has significantly 
higher oxidation resistance at high temperatures and requires less protective gases [6]. The new magnesium-calcium alloy is subsequently added to aluminum to produce a modified 7175 aluminum alloy with improved mechanical properties as a side-effect of the cleaner manufacturing process [7]. The mechanical properties are improved because the cleanliness of the process results in clean molten aluminum with greatly reduced oxides and inclusions through controlled oxidation of the magnesium during the melting, holding, transferring, pouring, and filling [4]. The lack of, or reduced usage of, expensive protective gases and the improved efficiency of the manufacturing process reduces the overall cost of production. Generally, silicon and iron are not desired in aluminum alloys as they decrease the fatigue strength of the material through the formation of $\mathrm{Al}_{7} \mathrm{Cu}_{2} \mathrm{Fe}$ and $\mathrm{Mg}_{2} \mathrm{Si}$ [8][9]. However, silicon and iron are present in the current specimens because they are impurities which are typically found in available commercial stock aluminum. They are expected in aluminum alloys with a maximum allowable composition of $0.15 \%$ for silicon and $0.20 \%$ for iron. The use of magnesium-calcium alloy also has the added benefit of reduced formation of $\mathrm{Mg}_{2} \mathrm{Si}$ caused by the presence of silicon in stock aluminum through controlled oxidation, and thus the resulting aluminum alloy has improved fatigue strength.

The differences between each experimental variant of ECO7175 lies in their composition. Their compositions are tabulated and shown in Table 1.1. The composition of standard reference 7175 is also included. 
Table 1.1: Manufactured composition of aluminum ECO7175 and standard reference aluminum 7175. Bold elements were intentionally added. Non-bold elements existed as natural impurities in commercial pure aluminum ingots. Compositions in wt\%.

\begin{tabular}{c|cccc}
\hline Element & ECO7175v1 & ECO7175v2 & ECO7175v3 & Ref.7175 \\
\hline $\mathrm{Al}$ & Balance & Balance & Balance & Balance \\
$\mathrm{Ca}$ & $\mathbf{0 . 0 4 1}$ & $\mathbf{0 . 0 2 5 2}$ & $\mathbf{0 . 0 3 1}$ & - \\
$\mathrm{Cr}$ & $\mathbf{0 . 2 1}$ & $\mathbf{0 . 2 2}$ & 0.002 & $\mathbf{0 . 1 8 - 0 . 2 8}$ \\
$\mathrm{Cu}$ & $\mathbf{1 . 7 4}$ & $\mathbf{1 . 3 7}$ & $\mathbf{1 . 2 8}$ & $\mathbf{1 . 2 - 2 . 0}$ \\
$\mathrm{Fe}$ & 0.08 & 0.08 & 0.07 & 0.20 \\
$\mathrm{Mg}$ & $\mathbf{2 . 7 3}$ & $\mathbf{2 . 3 4}$ & $\mathbf{2 . 4 2}$ & $\mathbf{2 . 1 - 2 . 9}$ \\
$\mathrm{Mn}$ & 0.01 & 0.01 & 0.01 & 0.10 \\
$\mathrm{Si}$ & 0.04 & 0.05 & 0.05 & 0.15 \\
$\mathrm{Ti}$ & $\mathbf{0 . 0 2 8}$ & $\mathbf{0 . 0 3 6}$ & $\mathbf{0 . 0 4 5}$ & 0.10 \\
$\mathrm{Zn}$ & $\mathbf{5 . 9 5}$ & $\mathbf{5 . 6 3 1}$ & $\mathbf{5 . 5 5}$ & $\mathbf{5 . 1 - 5 . 6}$ \\
\hline
\end{tabular}

\section{Problem Statement and Purpose}

The primary purpose of this study is to study the fatigue characteristics of this newly developed ECO7175 aluminum alloy. While the material's tensile behaviors are measured and known, very little else is known about the material. As there are interests in using this material as a structural material and for landing gear parts, there are interests in its fatigue characteristics. Currently, there are three experimental variants of the ECO7175 aluminum alloy: ECO7175v1, ECO7175v2, and ECO7175v3. It is desired to understand and compare the different variant's fatigue characteristics. The objectives are to subject the specimens to fatigue life testing, fatigue life data analysis, and observation of its fracture characteristics through optical microscopy and scanning electron microscopy (SEM), and metallography to study its grains and surface characteristics. With this knowledge, further development of 
the material can proceed and determined whether this material and the methods to produce it, is a suitable alternative to the standard 7175 aluminum alloy due to its improved properties and fatigue characteristics. 


\section{Chapter 2}

\section{Background Theory}

\subsection{Fatigue of Materials}

"Fatigue" in everyday use, means to be very tired and worn out. According to the Oxford English Dictionary, the meaning of "fatigue" is, "Extreme tiredness resulting from mental or physical exertion or illness." Extrapolating that definition to "metal fatigue," one could imagine a piece of metal studying all day and every day at university on how to be metal and is too tired and worn out to do its proper job as a pillar of support at home due to metal fatigue. This imagery gives a good idea of what is metal fatigue. According to the same Oxford English Dictionary, "metal fatigue" is, "Weakness in metal or other materials caused by repeated variations of stress." In a more general form, it is known as the "fatigue of materials" where a material undergoes progressive damage that causes weakening of the material until fracture due to repeated cyclic loading of stresses well below the material's ultimate tensile strength or yield strength [10]. In a wider sense, fatigue of materials also accounts for environmental stimulus such as fatigue of materials due to cyclic thermal loadings (i.e. those experienced by electronics and electrical components due to on-off and usage cycles). As such, fatigue of materials is a serious failure phenomenon that is of high importance in aerospace structures where it plays an important role in determining the reliability of an aircraft. Some of the other important characteristics of fatigue 
include the degree of randomness (stochastic) of fatigue in which seemingly identical components showing considerable scatter even in well controlled environment and how fatigue characteristics of the material can considerably be influenced by the material's microstructure, residual stresses within the material, presence of chemicals, surface finish, and more.

Unlike static failures, usually before the material or a particular component fail, there are clear indications of impending failures, such as very large deformations. The material or components are then quickly replaced or repaired. These characteristics of static failures allow quick detection through frequently scheduled inspections and audits. On the other hand, fatigue gives little to no visible indicators of impending failure and will often fail in a catastrophic manner. These particular characteristics of fatigue failures are what makes it incredibly dangerous due to its unpredictability. A real life example of a catastrophic fatigue failure that resulted in the death of around 52-200 deaths is the Versailles rail accident in France, also known as Catastrophe ferroviaire de Meudon in France, of 1842 on May 8th [11]. A train was returning from Paris, following the celebrations at the Palace of Versailles by King Louis Philippe I, between the Meudon and Bellevue station, derailed due to a fatigue failure of an axle in the leading locomotive. This axle failure leads to the crash and piling of the trailing carriages which were then lit on fire. During the time period, it was considered one of the deadliest railway accident in the world. This accident would then lead to systematic research into fatigue failure. Even with our modern understanding of 
fatigue failures, accidents due to fatigue still occurred throughout the world, such as in the 1998 Eschede derailment when a German high-speed ICE train suffered a fatigue failure from a single crack in its wheel. The failure caused the train to derail near the village of Eschede, subjected to subsequent series of multiple failures and crashes, finally destroying a bridge's support which caused the bridge to collapse on top of the train, and resulting in the death of 101 people. Fatigue failures were also common failures in United States Air Force (USAF) fighter's engines back in the 1990s [12].

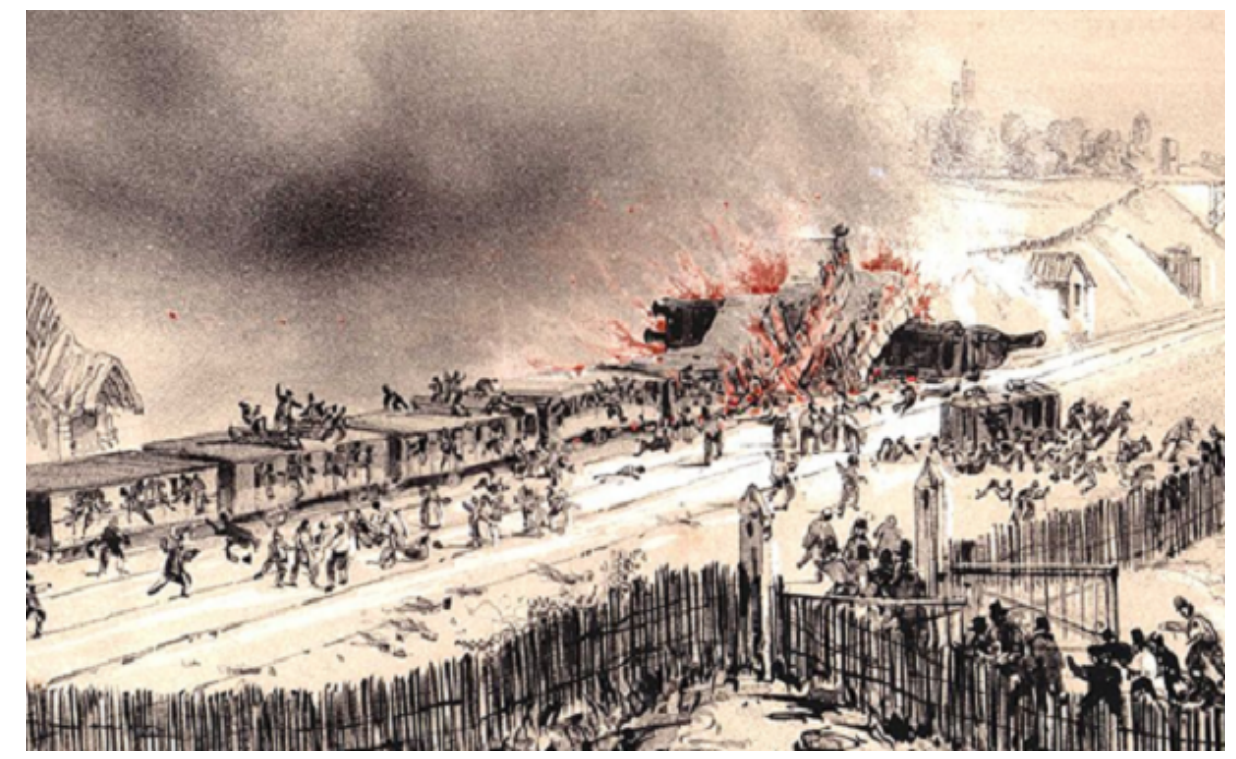

Figure 2.1: 1842 Versailles rail accident in France [13].

As stated previously, fatigue failures occur under cyclical loads which causes localized progressive structural damage to the material even when the stresses are significantly lower than the tensile strength and yield strength of the material. In aerospace applications, various components are frequently subjected to these cyclical 


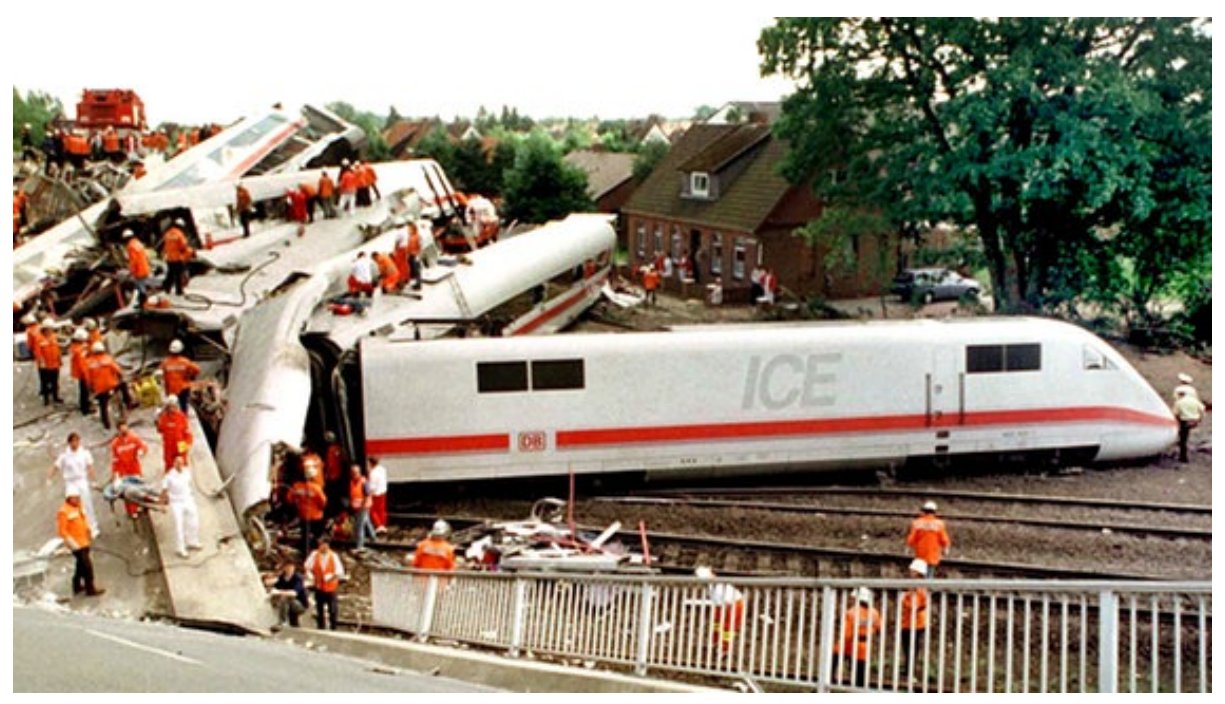

Figure 2.2: 1998 Eschede derailment in Germany [14].

loads at high frequencies. Therefore, it is imperative to know how many cycles these components can survive given that the applied stresses are known. The fatigue behavior of materials is described by an S-N curve, also known as the Wöhler curve, where $S$ is the stress amplitude of the cyclical stress and $N$ is the number of cycles to failure on a logarithmic scale. Stress amplitude, $\sigma_{a}$, is related to mean stress, $\sigma_{m}$, stress range, $\Delta \sigma$, and stress ratio, $R$, through the following equations (Fig. 2.3).

$$
\begin{gathered}
\Delta \sigma=\sigma_{\max }-\sigma_{\min } \\
\sigma_{a}=\frac{\sigma_{\max }-\sigma_{\min }}{2} \\
\sigma_{m}=\frac{\sigma_{\max }+\sigma_{\min }}{2} \\
R=\frac{\sigma_{\min }}{\sigma_{\max }}
\end{gathered}
$$




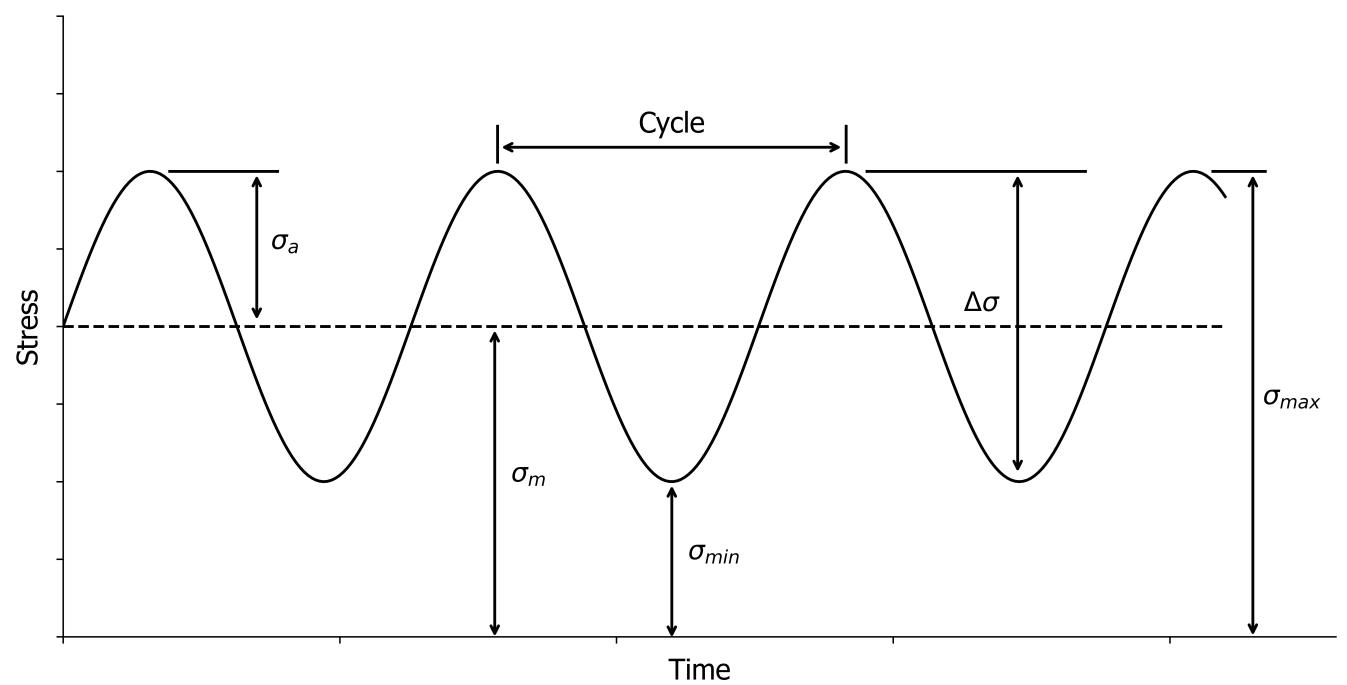

Figure 2.3: Fatigue Stress Parameters.

The one particular interest of the $\mathrm{S}-\mathrm{N}$ curve is the endurance limit, $\sigma_{e}$. The endurance limit is the assumed stress in which stresses below it will not produce a fatigue failure irrespective of how many stress cycles are applied (i.e. there is infinite life). In the simplest design case, it is highly desirable to keep stresses below the endurance limit. The endurance limit of most steels and copper alloys are approximately between $35 \%$ and $50 \%$ of its tensile strength. However, it is widely accepted that aluminum and many high strength steels do not exhibit an endurance limit [15]; instead, in the current context, aluminum has a fatigue strength at defined cycles to failure. In other words, given enough time and cycles, aluminum, high strength steel, and various materials, will eventually fail. In fact, according to more recent literature, there is no such thing as an endurance limit [16]. Given enough time, eventually, fatigue failure will occur in all materials at all stresses [17]. 


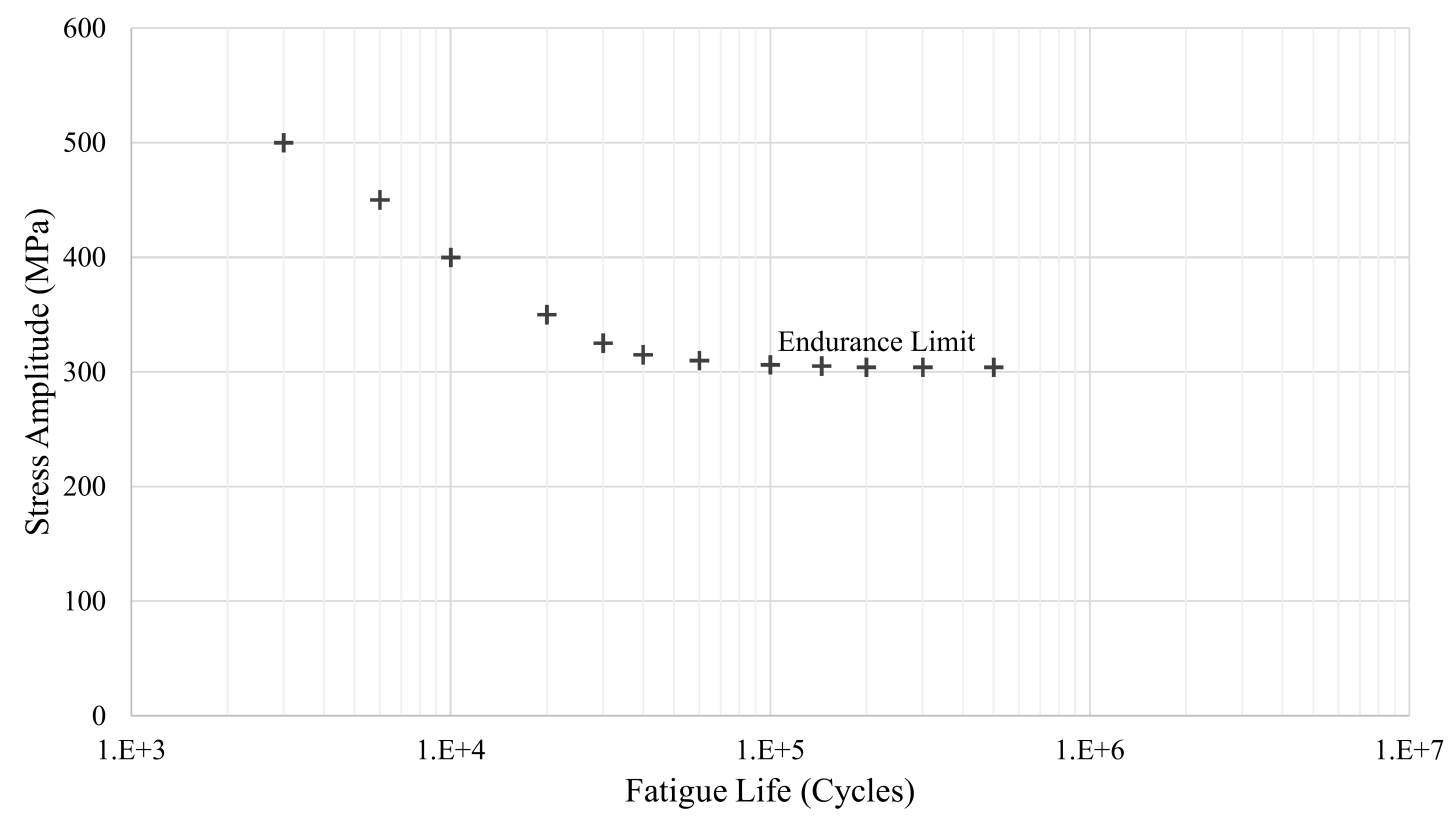

Figure 2.4: An example S-N curve with a material's endurance limit, $\sigma_{e}$, shown.

\subsection{Fatigue Analysis Approach}

In the analysis of the fatigue phenomena, there are several methods of approach. One method of approach is known as the stress-life approach, first introduced by August Wöhler in the 1860s. The stress-life approach, where the stress amplitude is held constant and controlled, is the first method of approach which was developed to understand and characterize fatigue of materials. The stress-life approach is an empirical method that has very widespread use in applications where the components are designed for long life (i.e. high-cycle fatigue $(\mathrm{HCF})$ ) where the primary induced deformations are elastic. On the other hand, the stress-life approach does not take into account adequately for applications where the primary deformations are plastic, induced by high stress amplitudes (low-cycle fatigue (LCF)). As a result, Coffin and 
Manson, independently, developed a characterization of fatigue that also takes into account plastic deformation in low-cycle fatigue. This characterization takes into consideration the total strain that accounts for both plastic strain and elastic strain. This is known as the Coffin-Manson relationship. This characterization is primarily used in the strain-life approach where the strain amplitude is held constant and controlled [18][19].

$$
\frac{\Delta \varepsilon}{2}=\frac{\sigma_{F}^{\prime}}{E}\left(2 N_{f}\right)^{b}+\varepsilon_{F}^{\prime}\left(2 N_{f}\right)^{c}
$$

where $\frac{\Delta \varepsilon}{2}$ is the total strain amplitude, $E$ is the modulus of elasticity (Young's modulus), $2 N_{f}$ is the reversals to failure ( 1 reversal to failure is equal to $1 / 2$ cycle to failure), $\sigma_{F}^{\prime}$ is the fatigue strength coefficient which represents the stress corresponding to fracture in one reversal, $b$ is the fatigue strength exponent, $\varepsilon_{F}^{\prime}$ is the fatigue ductility coefficient representing the strain corresponding to fracture in one reversal, and $c$ is the fatigue ductility exponent. The fatigue ductility coefficient, $\varepsilon_{F}^{\prime}$, is approximately equal to the strain to cause fracture in one reversal [20]. Similarly, the fatigue strength coefficient, $\sigma_{F}^{\prime}$, is approximately equal to the stress to cause fracture in one reversal [15]. The first component of the Coffin-Manson equation, $\frac{\sigma_{F}^{\prime}}{E}\left(2 N_{f}\right)^{b}$, represent the elastic strain component of the total strain. The second component of the CoffinManson relation, $\varepsilon_{F}^{\prime}\left(2 N_{f}\right)^{c}$, represent the plastic strain component of the total strain. In low-cycle fatigue (LCF), where cycles to failure are generally $10^{3}$ cycles or lower, plastic strain dominates. In high-cycle fatigue $(\mathrm{HCF})$, where cycles to failure are 
generally $10^{3}$ or higher, elastic strain dominates and therefore plastic strain can be assumed to be negligible. The material's fatigue life can then be approximated by equating the total strain to just the elastic strain component of the Coffin-Manson relation, Eq. 2.5. The Coffin-Manson relation can then simplify to:

$$
\frac{\Delta \varepsilon}{2}=\frac{\sigma_{F}^{\prime}}{E}\left(2 N_{f}\right)^{b}
$$

Eq. 2.6 can be further expanded and manipulated to become:

$$
\sigma_{a}=\frac{\Delta \sigma}{2}=\frac{\Delta \varepsilon}{2} E=\sigma_{F}^{\prime}\left(2 N_{f}\right)^{b}
$$

where $\frac{\Delta \sigma}{2}$ is the elastic stress amplitude. Eq. 2.7 is also known as the Basquin's equation [21]. The fatigue data can then be curve-fitted to the Basquin's equation to determine the fatigue strength coefficient, $\sigma_{F}^{\prime}$, and the fatigue strength exponent, $b$. Both of these constants are the material's unique properties. However, the Basquin's equation is only valid when the mean stress, $\sigma_{m}$, is zero. Morrow derived a modification of the equation which allowed the Basquin equation to work with any mean stress and is given in the following equation [22].

$$
\sigma_{a}=\frac{\Delta \varepsilon}{2} E=\left(\sigma_{F}^{\prime}-\sigma_{m}\right)\left(2 N_{f}\right)^{b}
$$

Cycles to failure, $N_{f}$, can be predicted given that the fatigue strength coefficient, $\sigma_{F}^{\prime}$, and the fatigue strength exponent, $b$, are known. Eq. 2.8 becomes: 


$$
N_{f}=\left(\frac{\sigma_{a}}{2\left(\sigma_{F}^{\prime}-\sigma_{m}\right)}\right)^{\frac{1}{b}}
$$

In the case of low-cycle fatigue, where plastic strain dominates, elastic strain can be assumed to be negligible. The material's fatigue life can then be approximated by equating the total strain to just the plastic strain component of the Coffin-Manson relation, Eq. 2.5. The Coffin-Manson relation can then simplify to:

$$
\frac{\Delta \varepsilon}{2}=\varepsilon_{F}^{\prime}\left(2 N_{f}\right)^{c}
$$

Experimental fatigue data can then be curve-fitted to the Eq. 2.10 to determine the fatigue ductility coefficient, $\varepsilon_{F}^{\prime}$, and the fatigue ductility exponent, $c$. Both constants are also the material's unique properties.

Finally, in the case where both plastic and elastic strain are significant, such as between $10^{3}$ and $10^{4}$ cycles, it may be desirable to use the Coffin-Manson relationship in its entirety for accurate characterization of the fatigue characteristics for the material.

\subsection{Reliability Analysis}

As fatigue failures are unexpected and catastrophic in nature, it is critical to conduct fatigue experiments for every material and components that are to be subjected to such loading. As a result, it is also important to understand and characterize the dependability of a material or a component in service, the probability that it will 
survive under fatigue loading. In a more general sense, it is important to understand and know the probability the component or material will perform its intended service for its designed service life [23]. To characterize a material's dependability, reliability analysis can be used. In reliability analysis, statistics and statistical distributions are important to understand. In probability theory, the probability distribution function (PDF), also known as the density curve, is a continuous function that specifies the probability of a random variable, $T$, falling within a range of values. The probability is given as the integral of the PDF over that range. Basically, the area under the curve between the range of interested values. An example of a PDF is given in Fig. 2.5 .

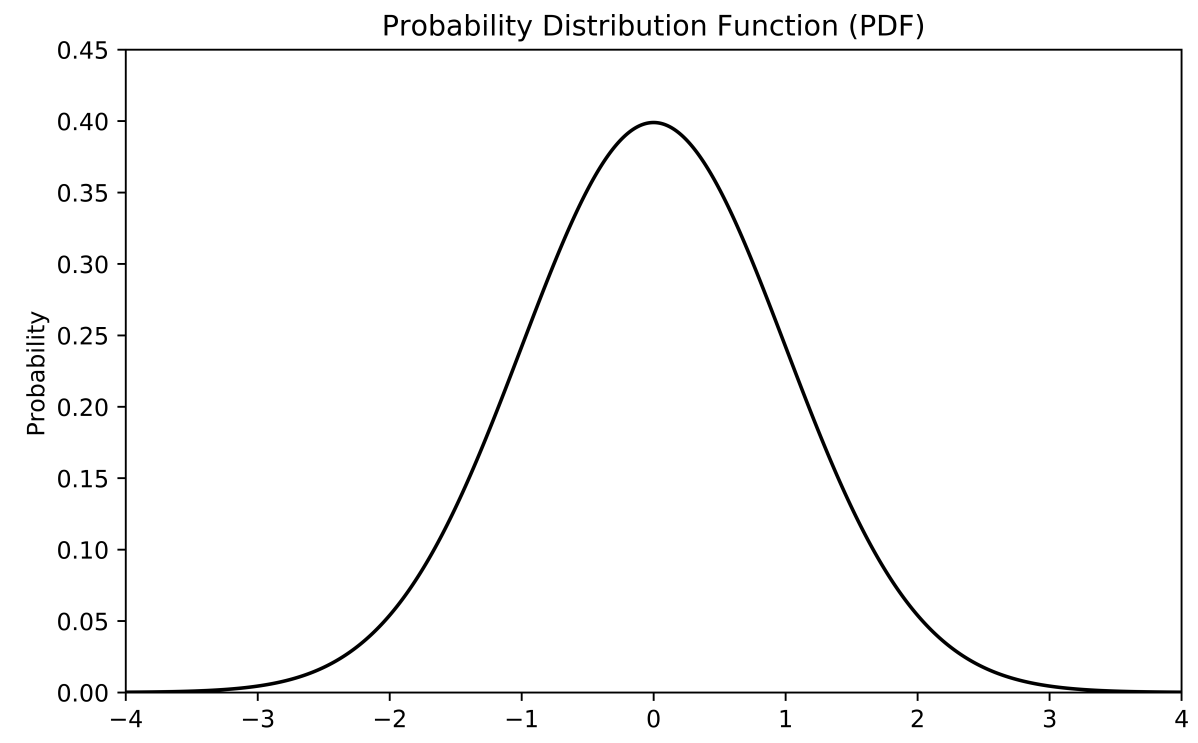

Figure 2.5: Probability Density Function (PDF).

Assuming $f(t)$ is the PDF, $T$ is a continuous random variable, and the interval $\left[t_{1}, t_{2}\right]$, or $[a, b]$, in which $a \leq b$, the PDF can be represented mathematically as the 
following:

$$
P(a \leq T \leq b)=\int_{a}^{b} f(t) d t
$$

where the probability of the random variable between interval $a$ and $b$ is equal to the area under the density curve between $a$ and $b$. As the area under the curve represents the probability, the cumulative area under the curve must be equal to 1 . As an example, using Fig. 2.5 where $a=-4$ and $b=4$, Eq. 2.11 becomes:

$$
P(-4 \leq T \leq 4)=\int_{-4}^{4} f(t) d t=1
$$

The cumulative distribution function $(\mathrm{CDF}), F(t)$, represent the area under the PDF to the left of $b$. In other words, the CDF represents the probability of a random variable that is equal to or less than $b$.

$$
F(t)=P(-\infty \leq T \leq b)=\int_{-\infty}^{b} f(t) d t
$$

An example of a CDF is given in Fig. 2.6. The complementary of the CDF (1-CDF) is also graphed in Fig. 2.6. The complementary of the CDF has a useful engineering meaning. In reliability engineering, a sub-discipline of engineering that focuses on the dependability of a product during its life cycle, the CDF is also known as the failure probability $F(t)$ that represents the probability a component or material will fail over its life [24]. The complementary to the failure probability is the reliability 


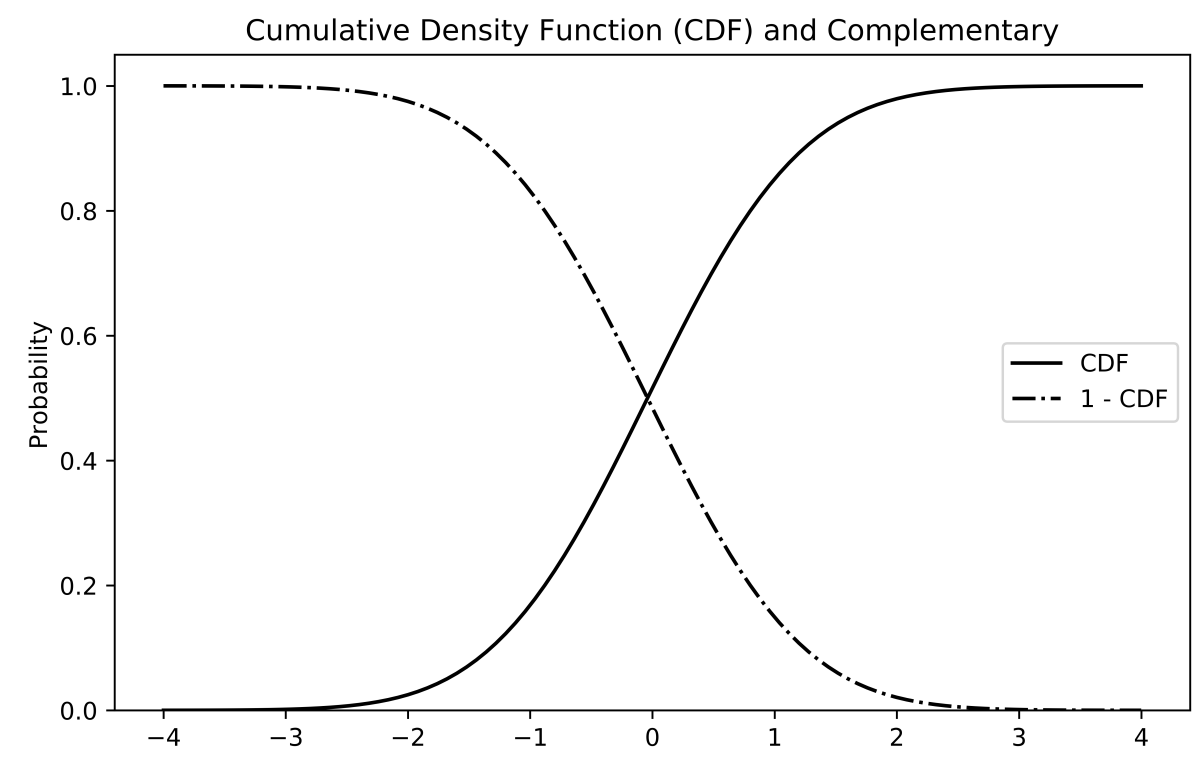

Figure 2.6: Cumulative density function (CDF) and its complement.

$R(t)$, which represent the probability a component or material will survive over its life. Reliability is also known as the survival function $S(t)$ outside of engineering. The PDF is also known as the failure density function $f(t)$ in reliability engineering. Using the new nomenclature, Fig. 2.6 becomes Fig. 2.7. Mathematically, the relationship between failure probability and reliability is shown in Eq. 2.14.

$$
R(t)+F(t)=1
$$

Another useful metric is the hazard rate, also known as the instantaneous failure rate or conditional failure rate. The hazard rate is defined as the probability that the component or material will fail at $t+\Delta t$ given that the component or material had survived up to time $t$. It is known as the conditional failure rate as it is the probability of the components that are still surviving $(1-F(t))$. The hazard rate is 


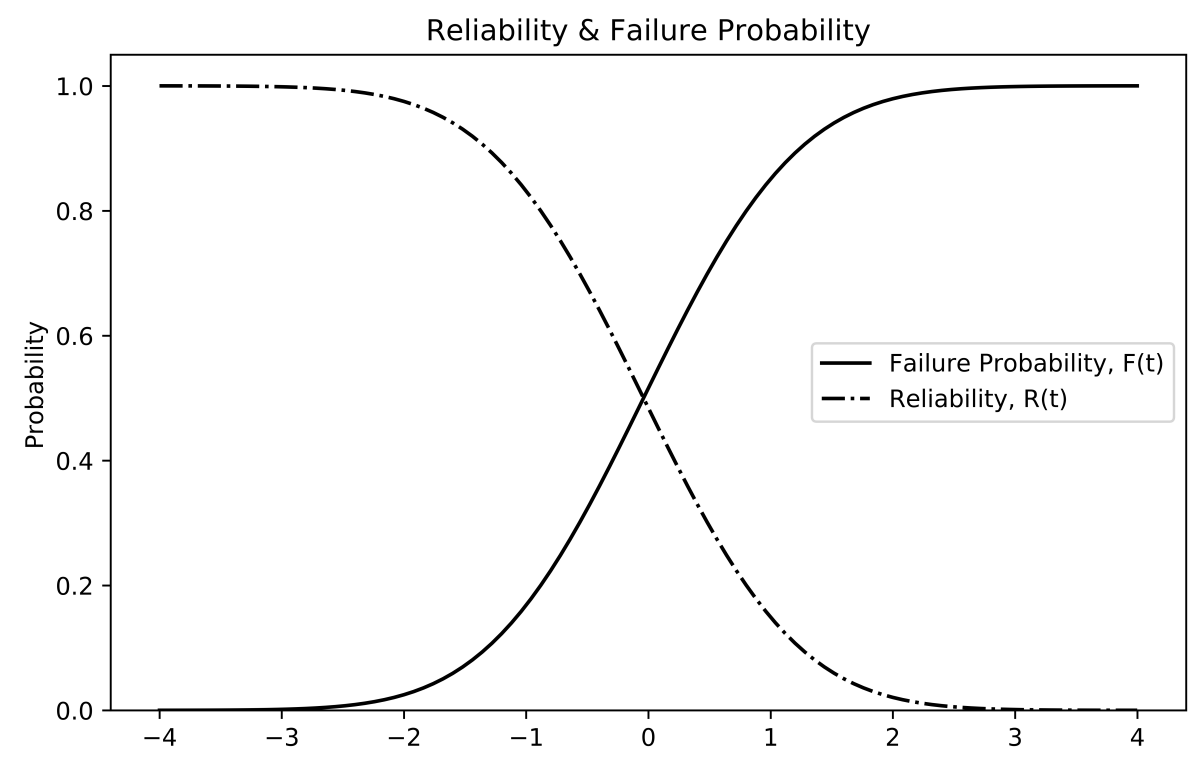

Figure 2.7: Failure probability $(\mathrm{CDF})$ and its complement, reliability.

given in Eq. 2.15.

$$
h(t)=\frac{f(t)}{1-F(t)}=\frac{f(t)}{R(t)}
$$

Understanding the hazard rate, it is possible to compare the observed hazard rate with a bathtub curve, a curve that is widely used in reliability engineering to determine whether a component or part is experiencing early failures, random failures, or wear-out failures [25]. An example of a bathtub curve is shown in Fig. 2.8. The curve is divided into three observations. Each observation is only seen individually and never all at once. The first observation, where the hazard rate is decreasing, represent the early failures which are also known as "infant mortalities." A real life analogy to infant mortalities is seen in burn-in tests for computer processors to weed out processors that are defective and will likely to die earlier than its designed life. 
The second observation of the curve is where the failure rate is constant. Continuing with the computer processors analogy, the processors that survived the burn-in test are then sold to consumers. The failures experienced by the consumers are random in nature over its designed life. This is also known as the processor's "useful life." As the consumer continues to use the processors over many years, eventually the processors will start to fail more frequently. These failures are increasing and are represented by the third observation of the bathtub curve. These processors are starting to become old and are dying due to wearing out.

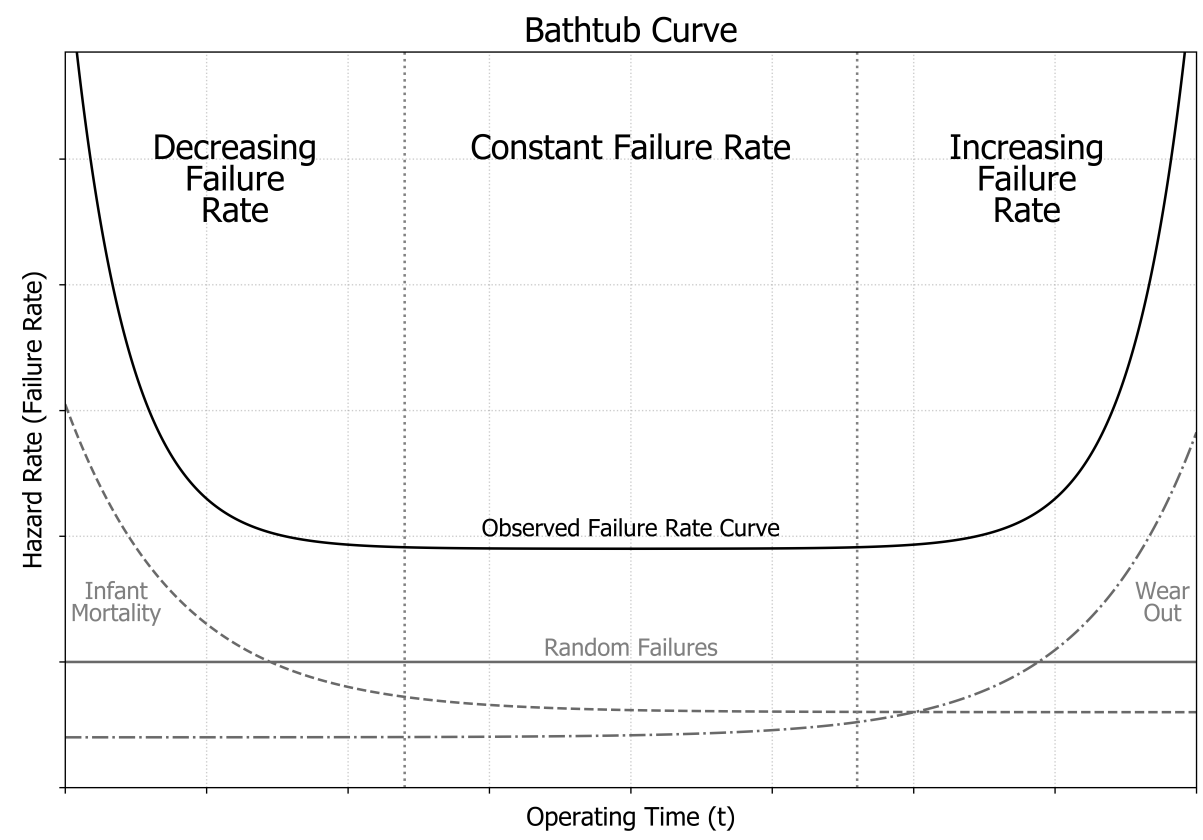

Figure 2.8: Bathtub curve of the hazard rate. 


\subsection{Estimating Reliability}

Generally, to calculate the reliability, the PDF and hazard rate would have to be calculated first. Alternatively, it is possible to estimate reliability directly using a non-parametric method that is simple in both their analysis and implementations. In addition, non-parametric methods have no reliance on an assumption that the resulting data are drawn from a given parametric family of probability distributions, such as the Weibull distribution. In other words, they allow the analysis of data without assuming an underlying distribution. They are extremely useful in studying any populations that are ranked, such as the star ratings on a product, as the ranking does not necessarily have a clear numerical interpretation. This gives non-parametric statistical methods robustness and a wider range of uses but in turn, there are situations where it will require larger samples sizes to draw conclusions with the same degree of confidence compared to parametric statistical methods.

The Kaplan-Meier estimator, widely used in the analysis of clinical data in the biomedical industries [26], is a non-parametric method that is used to estimate the survival function, known as reliability in engineering, from the given lifetime data [27]. It estimates the reliability by calculating the probability of each failure at the moment it occurred and can provide good estimates of the reliability of the component, given that the sample size is large enough. The Kaplan-Meier estimator is given in Eq. 2.16 . 


$$
\hat{R}_{t}=\hat{S}_{t}=\prod_{t_{i} \leq t}\left[1-\frac{d_{i}}{n_{i}}\right]
$$

$t_{i}$ is the time when at least one event, a specimen that has failed in fatigue, happened, $d_{i}$ is the number of events that occurred up to time $t_{i}$, and $n_{i}$ is the number of specimens that have yet failed at a time prior to $t_{i}$. In addition to the Kaplan-Meier estimator, the Nelson-Aalen estimator can be used to estimate the cumulative hazard function [28]. The cumulative hazard function is not the same as the hazard rate but estimating the cumulative hazard functions through the Nelson-Aalen estimator can give a good qualitative analysis of the hazard rates while remaining simpler to implement. Similar to the Kaplan-Meier estimator, the Nelson-Aalen estimator is a non-parametric statistical method that is frequently used in survival theory, life insurance, and reliability engineering to estimate the cumulative number of expected events. Similar to the Kaplan-Meier estimator, an "event" is described as a failure in engineering or deaths in life insurance and survival analysis. The Nelson-Aalen estimator is given in Eq. 2.17.

$$
\tilde{H}(t)=\sum_{t_{i} \leq t} \frac{d_{i}}{n_{i}}
$$

The minus of the natural logarithm of the survival function estimator (Eq. 2.17) given from the Kaplan-Meier estimator is equivalent to Eq. 2.18 [29] and can be implemented instead if the Kaplan-Meier estimator is to be used. 


$$
\hat{H}_{t}=-\ln \left(\hat{R}_{t}\right)
$$

While non-parametric estimators do not assume an underlying distribution model, if there are any indications that the estimator is following a distribution, more powerful predictions can be done by modeling the reliability curve to it. A common distribution in fatigue analysis is the Weibull distribution. The Weibull distribution for the CDF is presented in Eq. 2.19 .

$$
\begin{cases}1-e^{(t / \lambda)^{k}} & t \geq 0 \\ 0 & t<0\end{cases}
$$

where $\beta$ is the shape parameter and $\lambda$ is the scale parameter. As reliability is the complement of the CDF, as shown in Fig. 2.7, Eq. 2.19 becomes the reliability Eq. 2.20 .

$$
\begin{cases}R(t)=e^{(t / \lambda)^{\beta}} & t \geq 0 \\ 0 & t<0\end{cases}
$$

Eq. 2.18 is then used to determine the cumulative hazard function, $H(t)$. As the cumulative hazard function is simply the integral of the hazard rates from $t=0$ to $t=t$, the hazard rate, $h(t)$, can be determined. This implies that the cumulative hazard function and hazard rate for the Weibull distribution is of the form shown in Eq. 2.21. 


$$
\begin{aligned}
H(t) & =\left(\frac{t}{\lambda}\right)^{\beta} \\
h(t) & =\frac{\beta}{\lambda}\left(\frac{t}{\lambda}\right)^{\beta-1}
\end{aligned}
$$

Weibull distribution for the hazard rate (Fig. 2.9), hazard function (Fig. 2.10), reliability (Fig. 2.11), and its two parameters are demonstrated. As it can be seen in Fig. 2.9, the shape parameter, $\beta$, can be used to easily determine if a component or material is experiencing "infant mortalities" $(\beta<1)$, constant random failures $(\beta=1)$, or wear-outs $((\beta>1)$.

It is also important to have the necessary method to compare multiple reliability curves to determine whether there are significant differences between them. A logrank test (or logrank test), also known as a Mantel-Cox test, is a hypothesis test that can be used to determine if there are significant differences between reliability curves $[30][31][32]$. The null hypothesis and alternative hypothesis are as followed in Eq. 2.22 .

$$
\begin{aligned}
& H_{0}: h_{1}(t)=h_{2}(t) \\
& H_{A}: h_{1}(t)=c h_{2}(t), c \neq 1
\end{aligned}
$$

where $h_{1}(t)$ is the hazard ratio, the ratio of the estimated non-parametric hazard functions, of group $i$ at time $t$. If the calculated statistical $p$-value is less than or equal to the statistical significance, $\alpha$, the null hypothesis is rejected. If the calculated statistical $p$-value is more than the statistical significance, $\alpha$, the alternative 


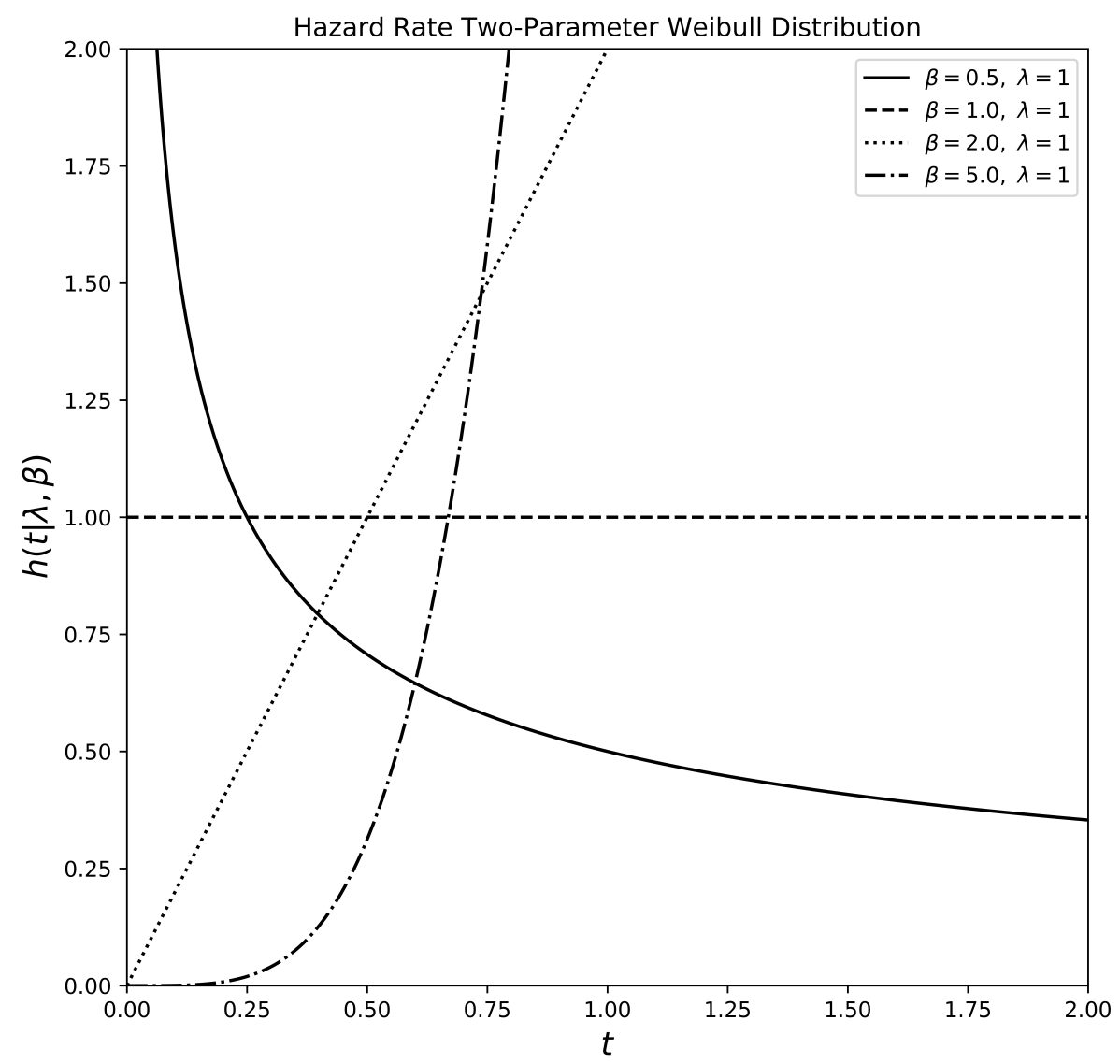

Figure 2.9: Weibull distribution for the hazard rate and its parameters. $\beta<1$ indicates early failures, $\beta=1$ indicates random failures, and $\beta>1$ indicates wearout failures.

hypothesis is rejected. However, for the log-rank hypothesis test to be used most effectively with the highest confidence, the proportional hazard assumption must be met. The proportional hazard assumption assumes that all reliability curves have the same hazard function, but with a unique scaling factor, $a_{i}$.

$$
h_{i}(t)=a_{i} h(t)
$$




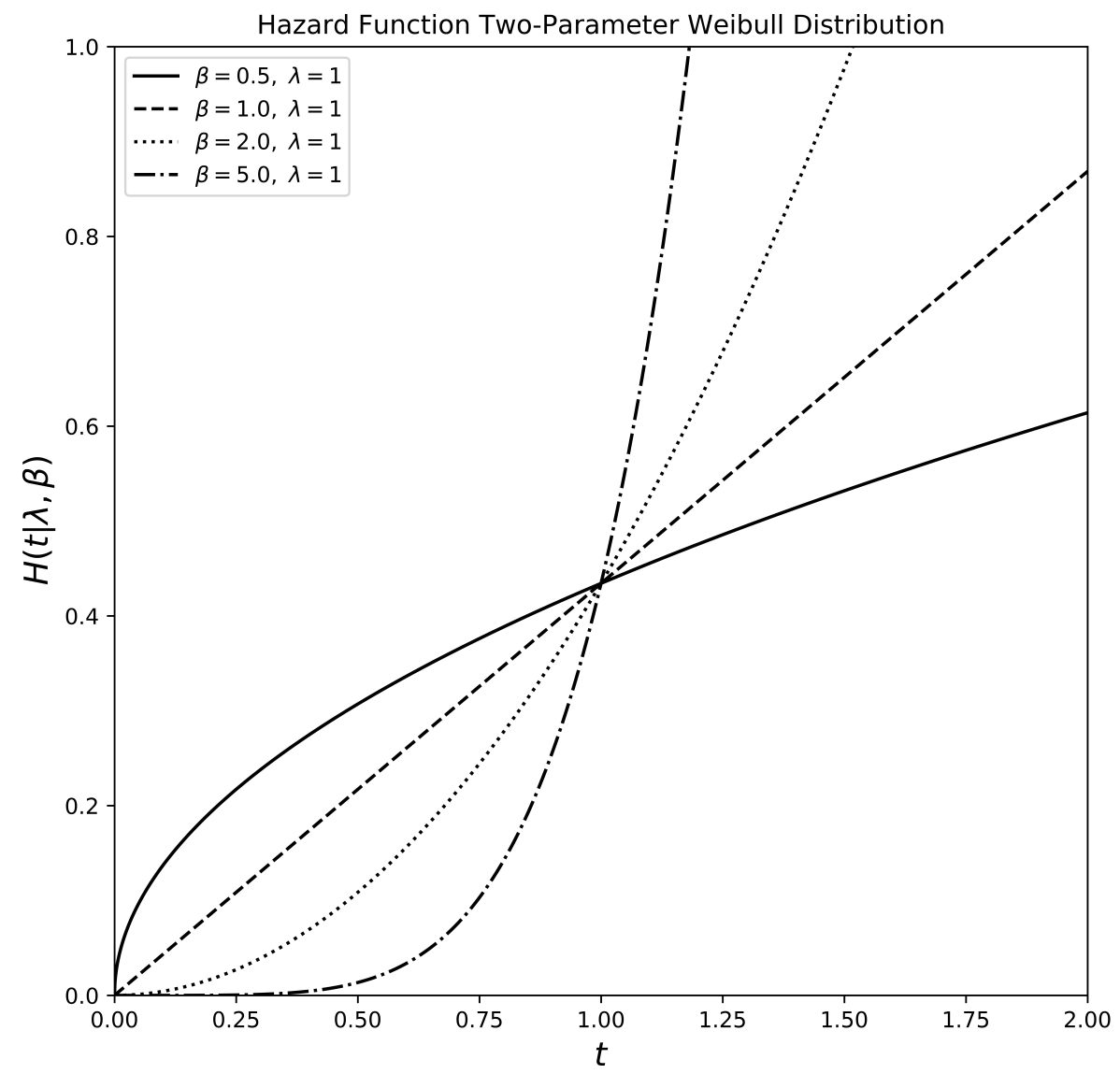

Figure 2.10: Weibull distribution for the cumulative hazard function and its parameters. Similarly to the hazard rate, $\beta<1$ indicates early failures, $\beta=1$ indicates random failures, and $\beta>1$ indicates wear-out failures. 


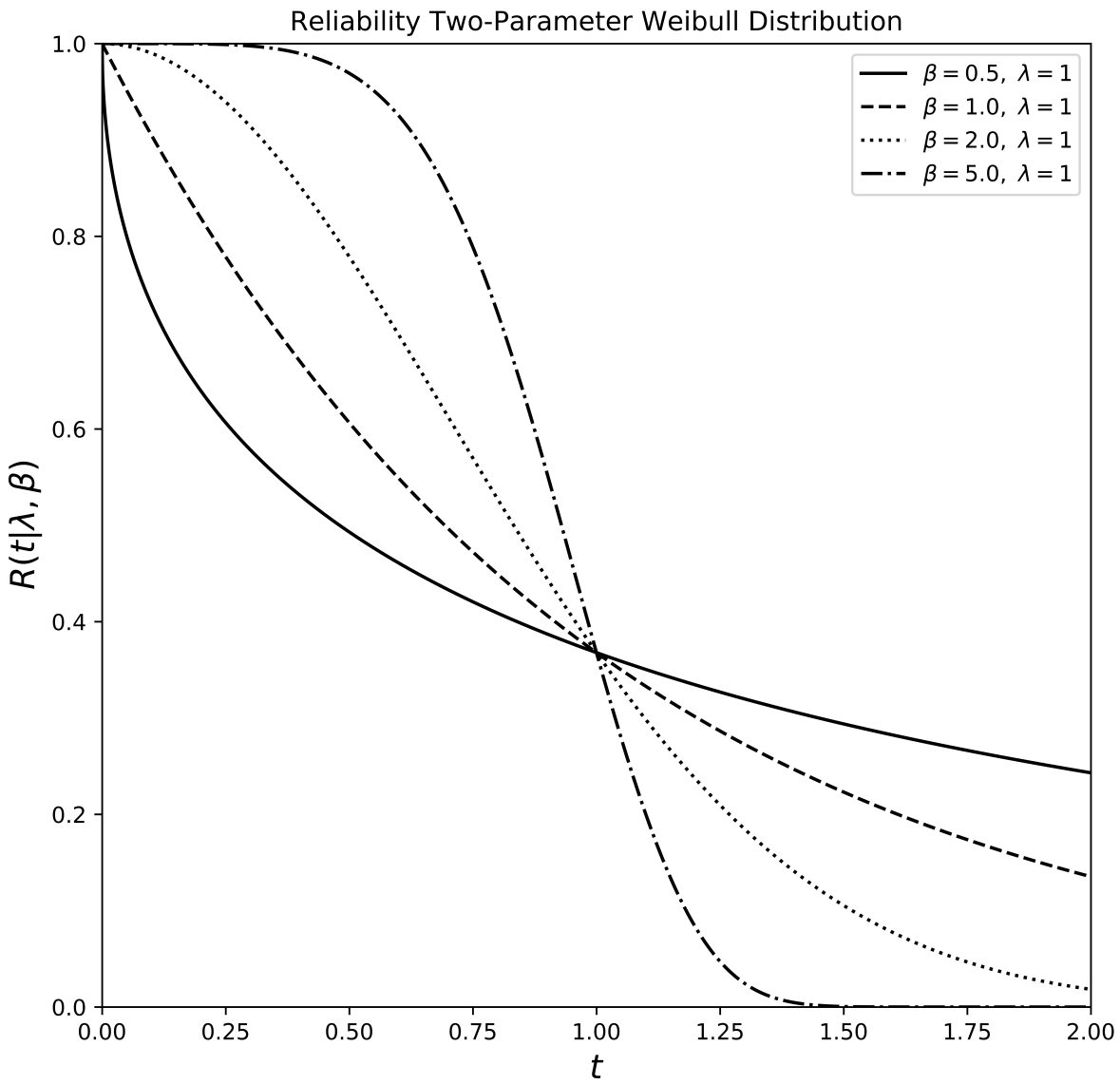

Figure 2.11: Weibull distribution for reliability and its parameters. 


\section{Chapter 3}

\section{Experimental Setup}

For this study, the fatigue stress-life approach was chosen and used to determine the fatigue strength of this new material under cyclic stresses. The fatigue stresslife approach was chosen due to its simplicity to implement with the equipment that was available with adequate accuracy for materials subjected primarily to elastic deformation. The specimens were in an hourglass-shape with the dimensions shown in Fig. 3.1.

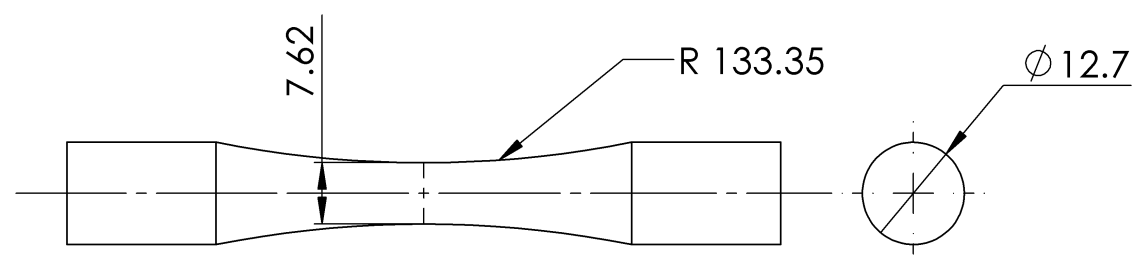

Figure 3.1: Fatigue coupon dimensions. Units are given in millimeter.

The stress amplitudes the specimens will be subjected to are chosen based on percentage of the ultimate tensile strength (UTS), such as $85 \%, 75 \%, 65 \%$, and so on. For ECO7175v1-T74, the stress amplitudes are chosen and calibrated are 86\%, 75\%, $66 \%, 55 \%, 44 \%, 34 \%$, and 24\%. For ECO7175v3-T74, the stress amplitudes chosen are $88 \%, 77 \%, 67 \%, 57 \%, 46 \%, 41 \%, 36 \%$, and $26 \%$. The specimens were allocated per chosen stress amplitude. Generally, in fatigue testing, the degree of randomness, or scatter, in cycles to failure increases with decreasing stress. In other words, there is a trend of increasing scatter for higher fatigue lives. Therefore, there is usually a need 
to allocate more specimens towards lower stress amplitudes to maintain a high level of confidence and reasonable variation in the data results. Due to limited specimens, the allocations were determined in such a way so that the desired $\leq 5 \%$ coefficient of variance with a confidence of $90 \%$ were maintained [33]. In determining the fatigue stress using the test equipment, the test specimens were subjected to a fully-reversed constant stress amplitude with stress ratio $R=-1$, in which the cyclic stress varied from tensile to compressive with equal magnitude. This study used a model RFB200 fatigue testing machine, manufactured by Fatigue Dynamics, Inc. (Fig. 3.2).

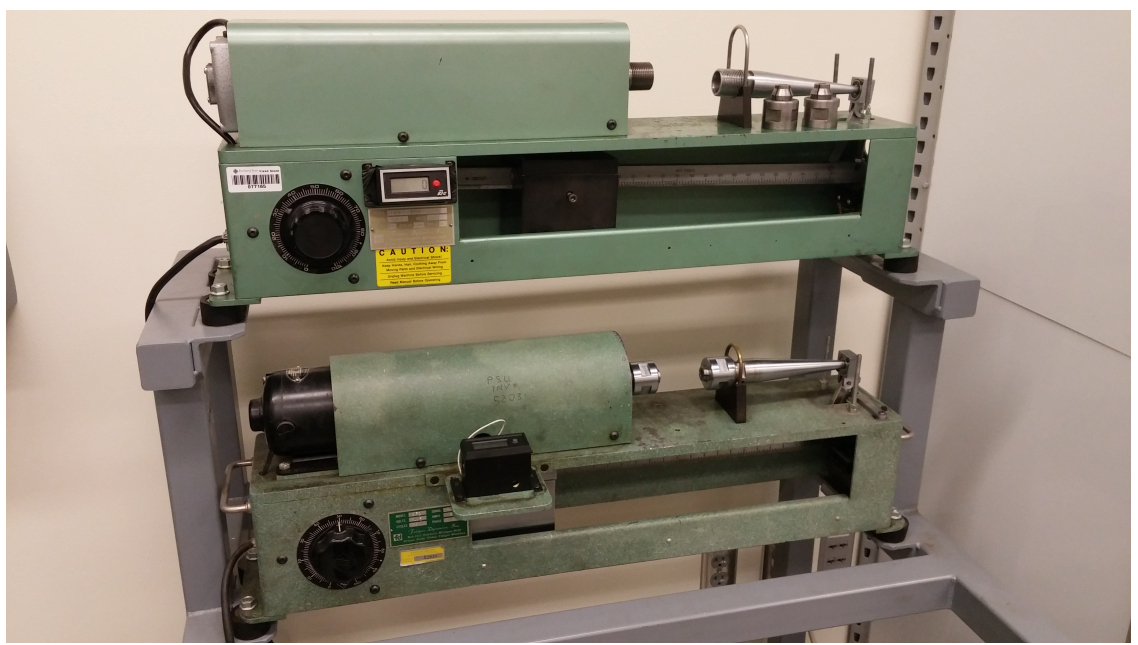

Figure 3.2: Model RFB200 fatigue tester by Fatigue Dynamics, Inc.

The motor speed for the fatigue tester was measured and verified using a NIST Traceable OMEGA HHT13 non-contact laser tachometer with a factory-tested certified accuracy of $\pm 0.01 \%$ of reading. To measure and calibrate the motor speed consistently, a specimen was wrapped in a dark tape to prevent false readings with the non-contact laser tachometer caused by the inherently shiny surface of the spec- 
imen. A small reflective tape is then attached on top of the dark tape which will then reflect the laser from the tachometer to allow speed measurement of the fatigue tester. The fatigue tester's settings for the applied moment loads was calibrated and verified using a Vishay Micro-Measurements rosette strain gauge. The rosette strain gauge was attached to a solid rod of a known material. The rosette strain gauge's signal is then conditioned, amplified, and read by Vishay Precision Group's Model P3 strain indicator. The applied moment load was then measured and compared to the setting on the fatigue tester.

Fatigue tests were conducted with the allocated number of specimens per load, run-out was defined as over $10^{7}$ cycles, as defined by the fatigue test conducted for aluminum AMS 4149 (7174-T74) in Metallic Materials Properties Development and Standardization (MMPDS) 11 [34]. However, for ECO7175v2-T74 and ECO7175v3T74's fatigue tests, run-out was changed and defined as above $5 \times 10^{7}$ cycles. This allows a better understanding of the fatigue strength of the material over a wider range. The change was done after the fatigue test for ECO7175v1-T74 was concluded.

The fracture surface of broken specimens was analyzed using both an Olympus SZX7 with a 5-megapixel camera and a Leica Microsystems' S6D StereoZoom optical microscope with a $0.75 x$ objective lens coupled to an AmScope MU900 9-megapixel digital camera through a Leica Microsystems' 1.0x optical phototube. Further analyses of the surface of broken specimens were then done using a Zeiss Sigma VP FEG scanning electron microscope (SEM). Compositional analysis through EDX (Energy- 
dispersive X-ray spectroscopy) were done using Oxford Instruments X-Max 50mm with Aztec 3.2 software package. Specimens prepared for SEM and metallography were sectioned using Struers' Accutom-50 with a silicon-carbide (SiC) blade, mounted with Struers' LabPress-3 using semi-conductive mounting powder which reduces overcharging issues in the SEM, and polished with Struers' automated polishing system consisted of the RotoCom, Multidoser, and RotoPol-31. Specimens were then etched using modified Keller's reagent which consisted of $2 \mathrm{ml} \mathrm{HF}$ (48\% concentrated), $3 \mathrm{ml}$ $\mathrm{HCl}$ (100\% concentrated), $20 \mathrm{ml} \mathrm{HNO}_{3}$ (100\% concentrated), and $175 \mathrm{ml}$ de-ionized water for approximately 10 seconds and then immediately dipped in de-ionized water to stop the etching process, rinse under fresh de-ionized water, and then blow dried [35]. The etched and polished surface of fatigue specimens were observed under the Olympus PMG3 inverted metallurgical microscope that is coupled to a PAX-it imaging camera with software. The metallurgical microscope also has support for Nomarski microscopy. All image processing was done using Fiji, an image processing package that is an alternative distribution of ImageJ. Anaconda 2018.12 with Python 3.7.2 was used for data analysis. Reliability analysis was also done using Anaconda 2018.12 but complemented with lifelines 0.19 .4 , a survival analysis library implemented in Python [36]. 


\section{Chapter 4}

\section{ECO7175v1-T74}

Table 4.1 shows the allocation of specimens for fatigue testing of ECO7175v1 to maintain the desired $\approx 5 \%$ coefficient of variation with a confidence of $90 \%$. The composition of ECO7175v1 is given in Table 4.2. ECO7175v1's tensile strength was determined through tensile tests. Table 4.3 compare its tensile properties with those of several other variants of aluminum 7175. Aerospace Material Specification (AMS), AMS 4149, and AMS 4344 are fabrication specifications for the aluminum 7175 alloy. AMS 4149 aluminum is manufactured through forging (die forging or hand forging) and subjected to T74 temper. AMS 4344 aluminum is manufactured through extrusion and subjected to T73511 temper. T74 temper indicated the alloy is tempered with the base $\mathrm{T} 7$ temper, in which the alloy is solution heat treated, quenched, and then overaged in a furnace (Fig. 4.1). The second digit, 4, in 74 indicated that further treatment is used to further enhance the strength, toughness, fracture toughness, and corrosion resistance [37]. T73511 temper is a variation of the T73 temper, in which the base temper is $\mathrm{T} 7$, with further treatment to increase stress-corrosion resistance of the alloy.

The experimental fatigue test results are tabulated in Table 4.4. Fatigue test results for stress amplitudes of 510,448, and $331 \mathrm{MPa}$ have a coefficient of variation of more than $5 \%$. Because of the limited number of specimens, it was decided to allocate 
Table 4.1: Allocation of Specimens for ECO7175v1-T74.

\begin{tabular}{cc}
\hline Stress Amplitude (MPa) & Number of Specimens \\
\hline 510 (86\% UTS) & 10 \\
434 (75\% UTS) & 10 \\
393 (66\% UTS) & 10 \\
331 (55\% UTS) & 13 \\
$262(44 \%$ UTS $)$ & 10 \\
$200(34 \%$ UTS $)$ & 5 \\
$138(24 \%$ UTS $)$ & 3 \\
\hline
\end{tabular}

Table 4.2: Manufactured Composition of Aluminum ECO7175v1-T74.

\begin{tabular}{cc}
\hline Element & Composition \% \\
\hline Aluminum $(\mathrm{Al})$ & Balance \\
Calcium $(\mathrm{Ca})$ & 0.041 \\
Chromium $(\mathrm{Cr})$ & 0.21 \\
Copper $(\mathrm{Cu})$ & 1.74 \\
Iron $(\mathrm{Fe})$ & 0.08 \\
Magnesium $(\mathrm{Mg})$ & 2.73 \\
Manganese $(\mathrm{Mn})$ & 0.01 \\
Silicon $(\mathrm{Si})$ & 0.04 \\
Titanium $(\mathrm{Ti})$ & 0.028 \\
Zinc $(\mathrm{Zn})$ & 5.95 \\
\hline
\end{tabular}

more experimental specimens to these stress amplitudes to bring the coefficient of variation closer to $5 \%$. After the allocation, the coefficient of variation for specimens loaded at 510 and $331 \mathrm{MPa}$ was still considerably higher than $5 \%$, but considerably lower than previously. 
Table 4.3: Tensile Properties of Aluminum 7175.

\begin{tabular}{ccccc}
\hline Variant & TYS (MPa) & UTS (MPa) & EL. (\%) & Reference \\
\hline AMS 4149 & 441 & 510 & 7 & 7175-T74 (die forge) [34] \\
AMS 4149 & 434 & 503 & 9 & 7175 -T74 (hand forge) [34] \\
AMS 4344 & 407 & 476 & - & 7175 -T73511 (extrude) [34] \\
ECO7175v1 & $489 \pm 13.6$ & $574 \pm 5.2$ & $10.5 \pm 0.47$ & Extrusion, T74 \\
ECO7175v2 & $516 \pm 0.6$ & $567 \pm 0.2$ & $10.5 \pm 0.03$ & Extrusion, T74 \\
ECO7175v3 & $499 \pm 1.6$ & $550 \pm 2.5$ & $12.5 \pm 0.37$ & Extrusion, T74 \\
\hline
\end{tabular}

Note: TYS $=$ tensile yield strength; UTS $=$ ultimate tensile strength;

$\mathrm{EL}=$ total elongation

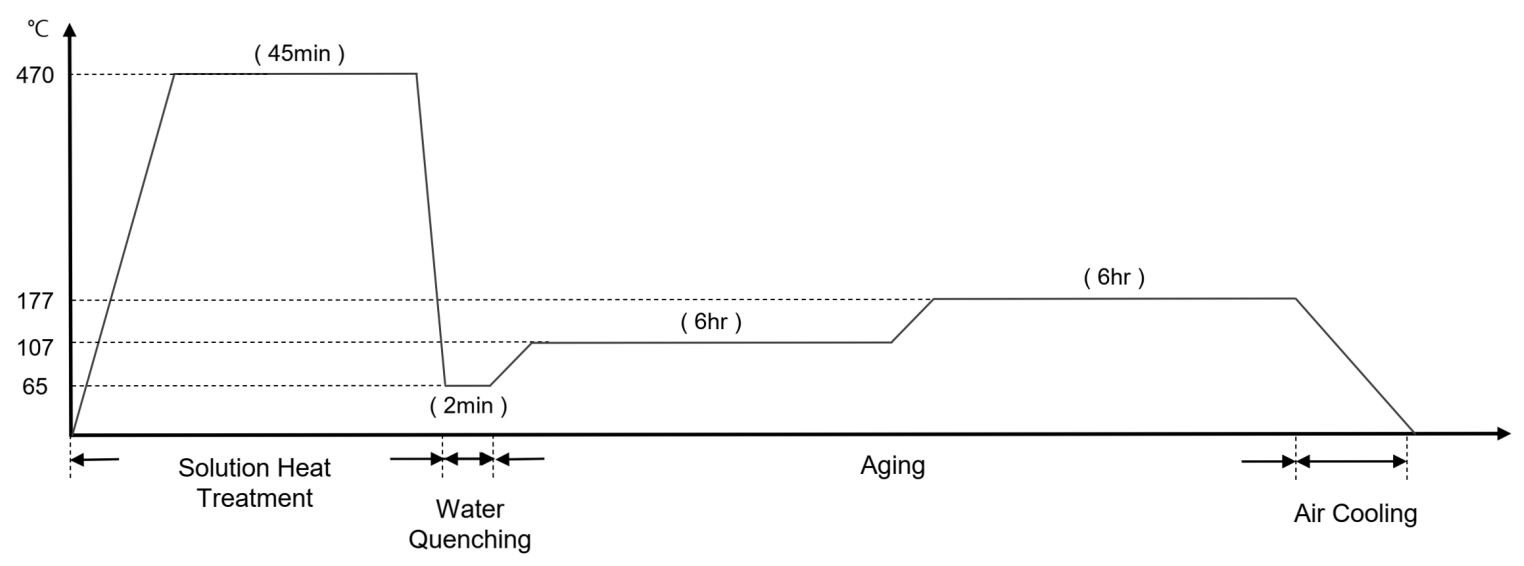

Figure 4.1: Heat treatment process for aluminum T74 temper.

Fig. 4.2 show the S-N curve for ECO7175v1. The S-N curve ranges from $10^{3}$ to $10^{7}$ cycles. The majority of the cycles to failure above $207 \mathrm{MPa}$ are between $10^{3}$ and $10^{6}$ cycles. The fatigue strength of the material at $10^{7}$ cycles is $207 \mathrm{MPa}$, approximately $36 \%$ of its tensile strength of $574 \mathrm{MPa}$.

There were no other data that could be compared directly with the fatigue test results of the new ECO7175v1 alloy. The closest data to be compared with would be of AMS 4149 (7175-T74), which is unnotched and hand forged as given by the 
Table 4.4: Statistical Analysis of Fatigue Testing for ECO7175v1-T74.

\begin{tabular}{c|ccc}
\hline Stress Amplitude (MPa) & Avg. Log Cycles & St.D. & Coeff. of Variation \% \\
\hline 510 (86\% UTS) & 3.581 & 0.216 & 6.0 \\
448 (75\% UTS) & 3.856 & 0.210 & 5.4 \\
393 (66\% UTS) & 4.159 & 0.180 & 4.3 \\
331 (55\% UTS) & 4.641 & 0.362 & 7.8 \\
262 (44\% UTS) & 5.041 & 0.158 & 3.1 \\
200 (35\% UTS) & \multicolumn{3}{c}{ Run-outs (Over 10 Cycles) } \\
138 (24\% UTS) & \multicolumn{3}{c}{ Run-outs (Over 10 $10^{7}$ Cycles) } \\
\hline
\end{tabular}

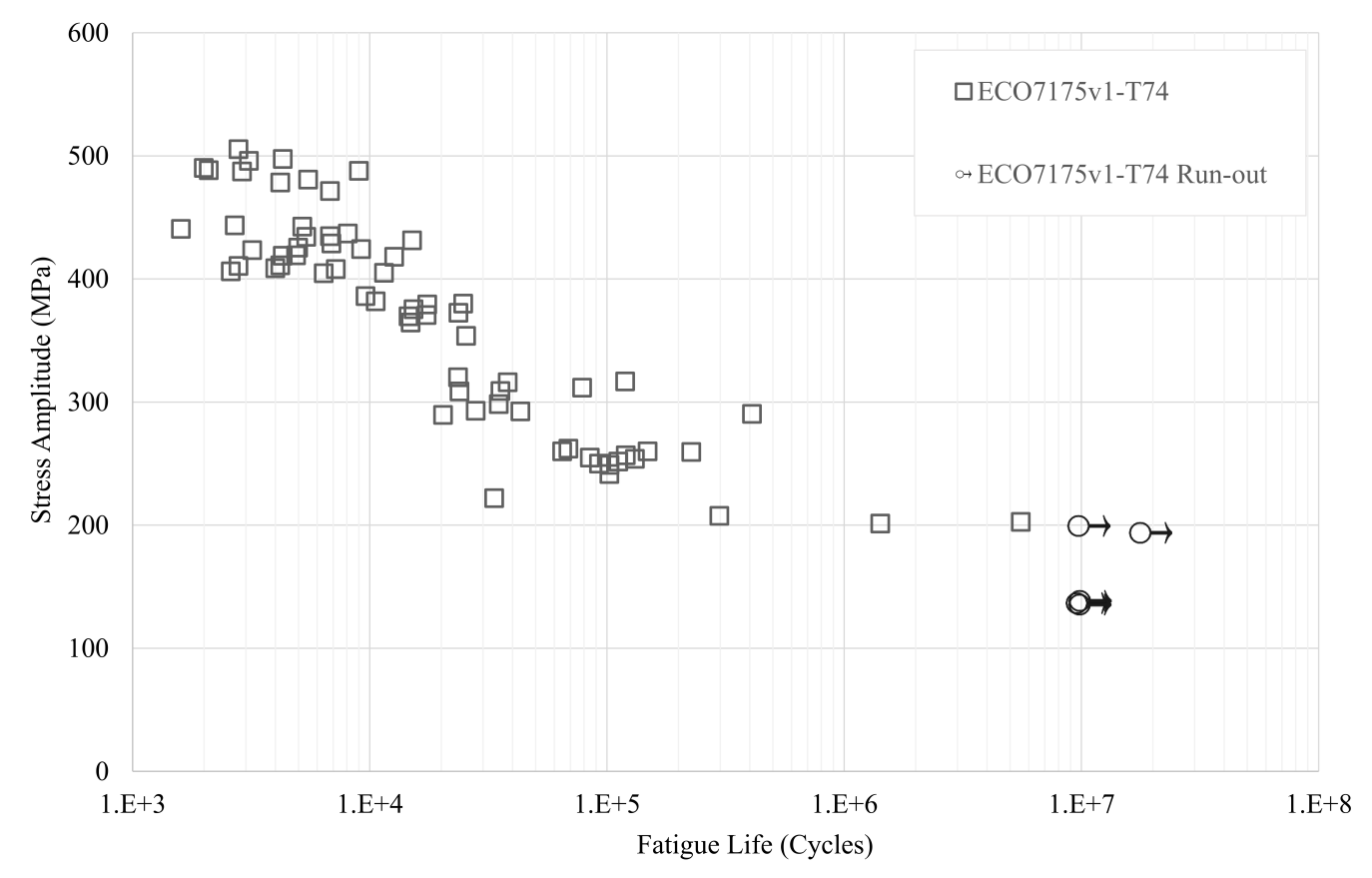

Figure 4.2: S-N data plot of aluminum ECO7175v1-T74.

MMPDS-11 handbook. However, there are many differences in the specimen geometry, fabrication process, and the testing method. ECO7175v1's minor diameter is 0.30 
in, compared with 0.25 in for AMS 4149. AMS 4149 is forged instead of extruded. ECO7175v1 was subjected to fully reversed bending loads with a stress ratio $R=-1$ rather than an axial load with stress ratios $R$ of 0.10 and 0.50 , as in the case for AMS 4149. All these differences make comparison results not viable.

\subsection{Basquin's Equation}

As the stress amplitudes for the fatigue tests are considerably below ECO7175v1's yield strength, the majority of the specimens' cycles to failure are within high cycle fatigue life. In high cycle fatigue life, a material's fatigue life is dominated by elastic strain and therefore its plastic strain can be assumed to be negligible. The fatigue data was then fitted to Basquin's equation to determine its constants (Fig. 4.3). The fatigue strength coefficient, $\sigma_{F}^{\prime}$, was determined to be 1557.6 MPa and the fatigue strength exponent, $b$, was determined to be -0.145 . 


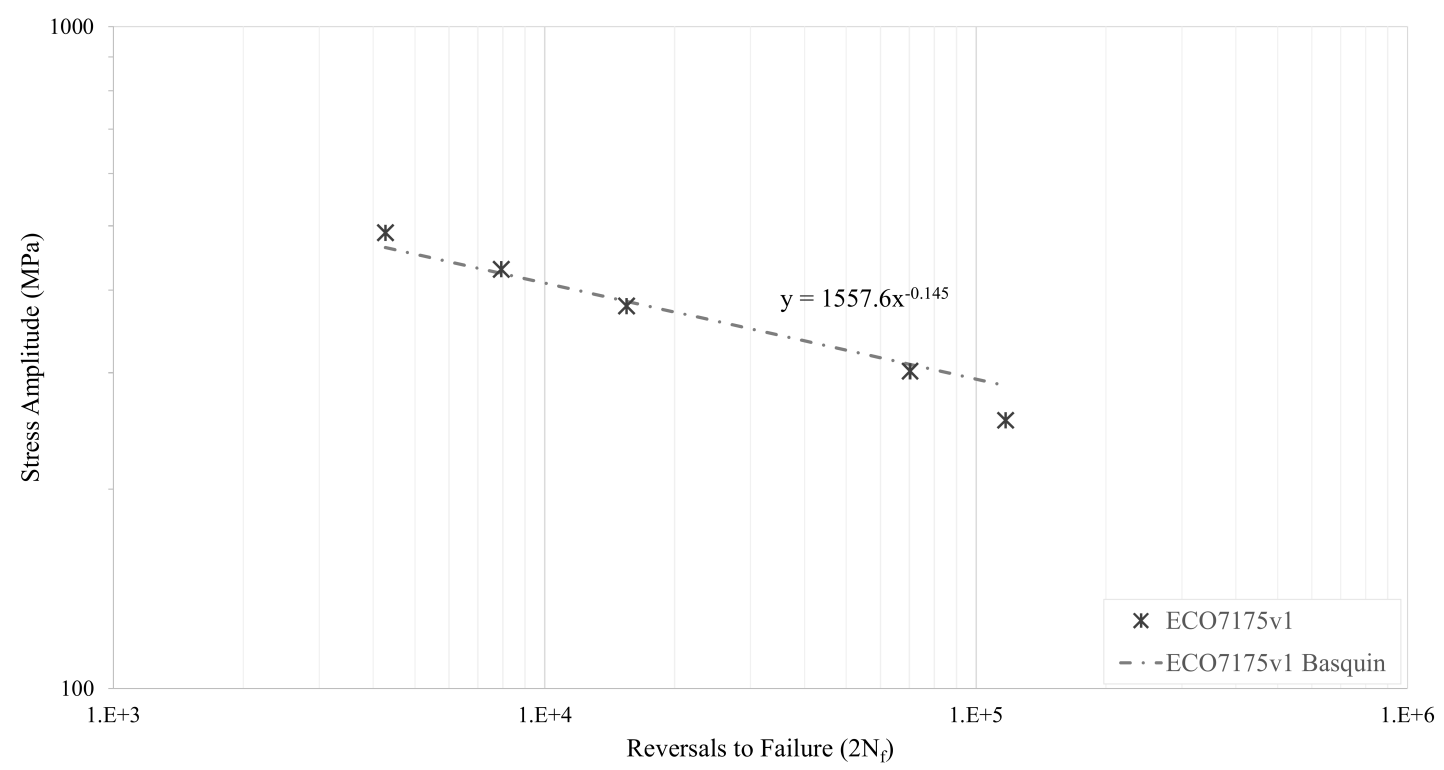

Figure 4.3: Fatigue data for ECO7175v1-T74 [38] curve-fitted to Basquin's equation to determine the material's fatigue strength coefficient, $\sigma_{F}^{\prime}$, and fatigue strength exponent, $b$.

\subsection{Fracture Analysis}

Fatigue fracture surfaces between specimens that were running at higher stresses and those at lower stresses were compared. Specimens that were subjected to relatively higher stress amplitudes, such as those in Fig. 4.4, which were subjected to $86 \%$ ultimate tensile strength (UTS), have relatively more fracture initiation points at the surface of the specimens. The fatigue crack propagation bands are relatively short compared with those from specimens subjected to lower stress amplitudes (Fig. 4.5). Fatigue specimens that were subjected to relatively lower stress amplitudes, such as those in Fig. 4.5, have relatively fewer crack initiation points. Instead, they typically have longer crack propagation bands. These crack propagation bands are 
capable of reaching halfway or more through the specimens. Fatigue specimens which were subjected to intermediate stress amplitudes, such as those subjected between $75 \%$ and $55 \%$ of ECO7175v1's tensile strength in Fig. 4.6, have mixed characteristics.

Generally, fatigue cracks initiate at the surface of specimens due to slip deformation or surface defects, which is the dominant form of fracture initiation. However, crack initiations at the subsurface are typically found at low stresses and high cycles for high-strength alloys in which inclusions are caused by impurities or are part of manufacturing processes, such as carburization for case-hardening [39][40][41][42]. Conversely, crack initiation sites appeared to mainly occur at the surface of the ECO7175v1 specimens rather than the subsurface (Fig. 4.7). This may be attributed to the fact that the specimens were seemingly free of inclusions. In all cases, the specimens have a relatively uniform and clean surface as there are no clear indications of inclusions or any sort of contamination from oxides or other inter-metallic compounds. This is the result of the new composition with the inclusion of calcium through the use of magnesium-calcium alloy. This resulted in a cleaner manufacturing process and thus, reduced oxides and other contaminants that are typically introduced into the bulk material due to the reactivity of magnesium.

Striations were found and measured. The striations, on average, have a width of approximately $0.481 \mu \mathrm{m}$, as shown in Fig. 4.8 . 

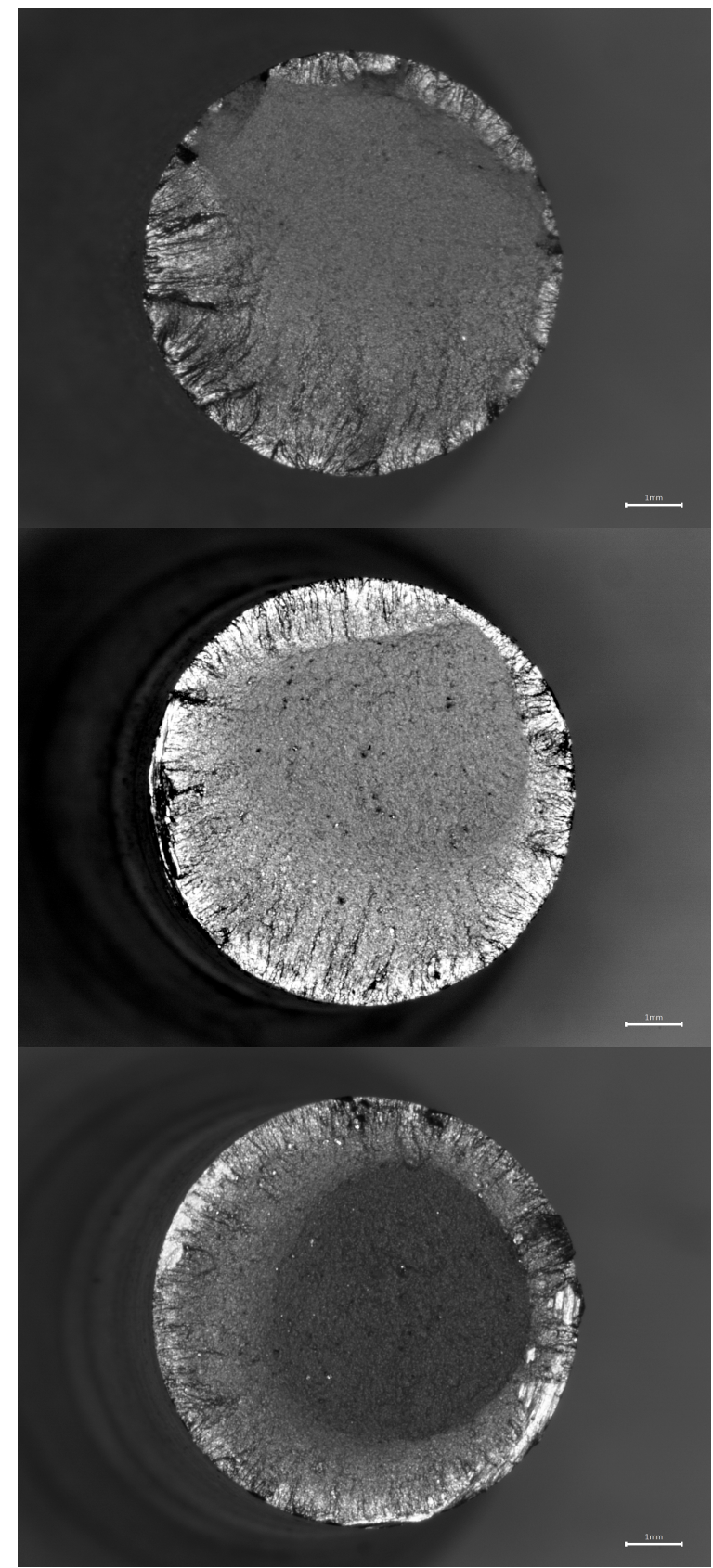

Figure 4.4: Specimens subjected to $86 \%$ UTS. $1 \mathrm{~mm}$ scale bar. Specimens at relatively higher stresses have more crack initiations points at the surface compared with specimens at relatively lower stresses. The crack initiation bands that propagate from these crack initiation points are also relatively shorter. 


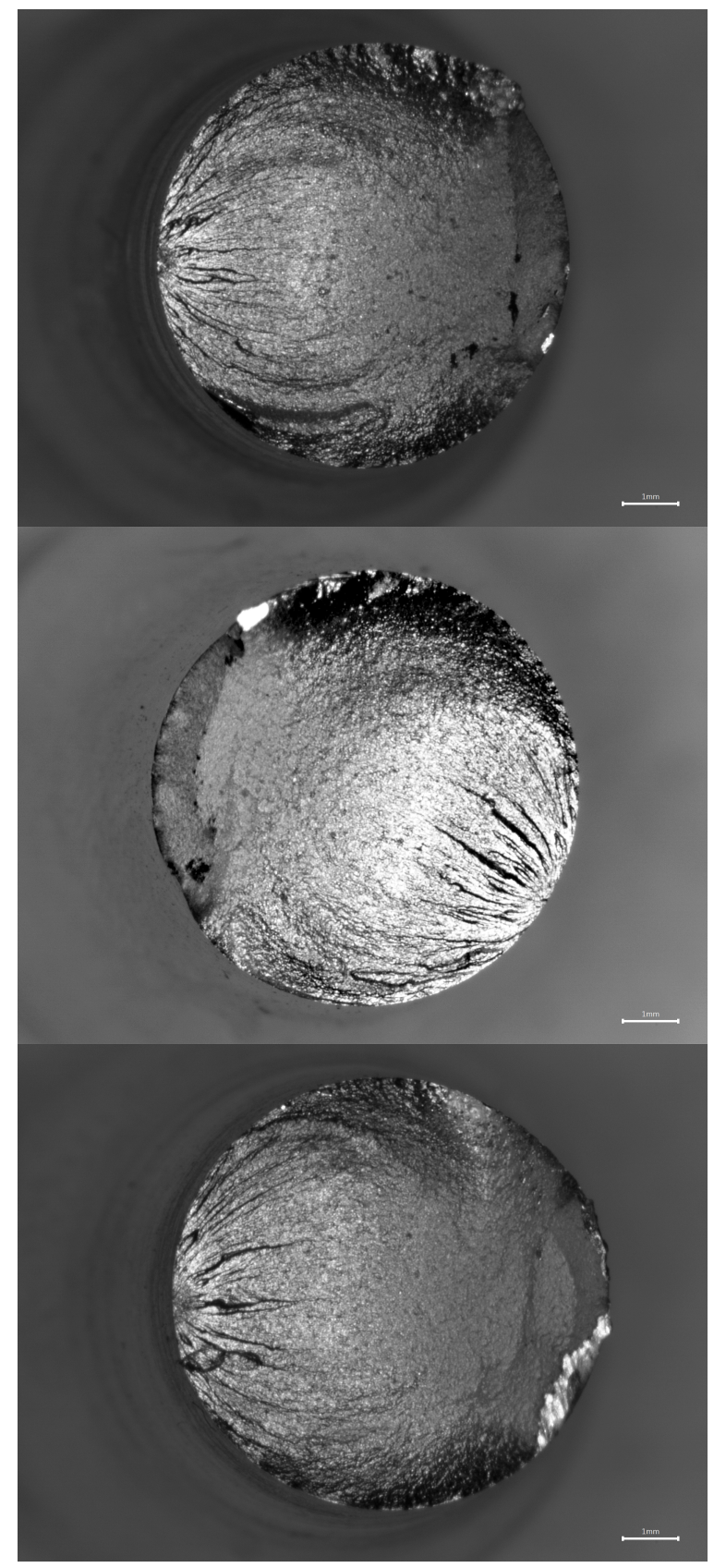

Figure 4.5: Specimens subjected to 44\% UTS. $1 \mathrm{~mm}$ scale bar. Specimens at lower stresses typically have fewer crack initiation points but typically have longer crack propagation bands that originate from these crack initiation points. 


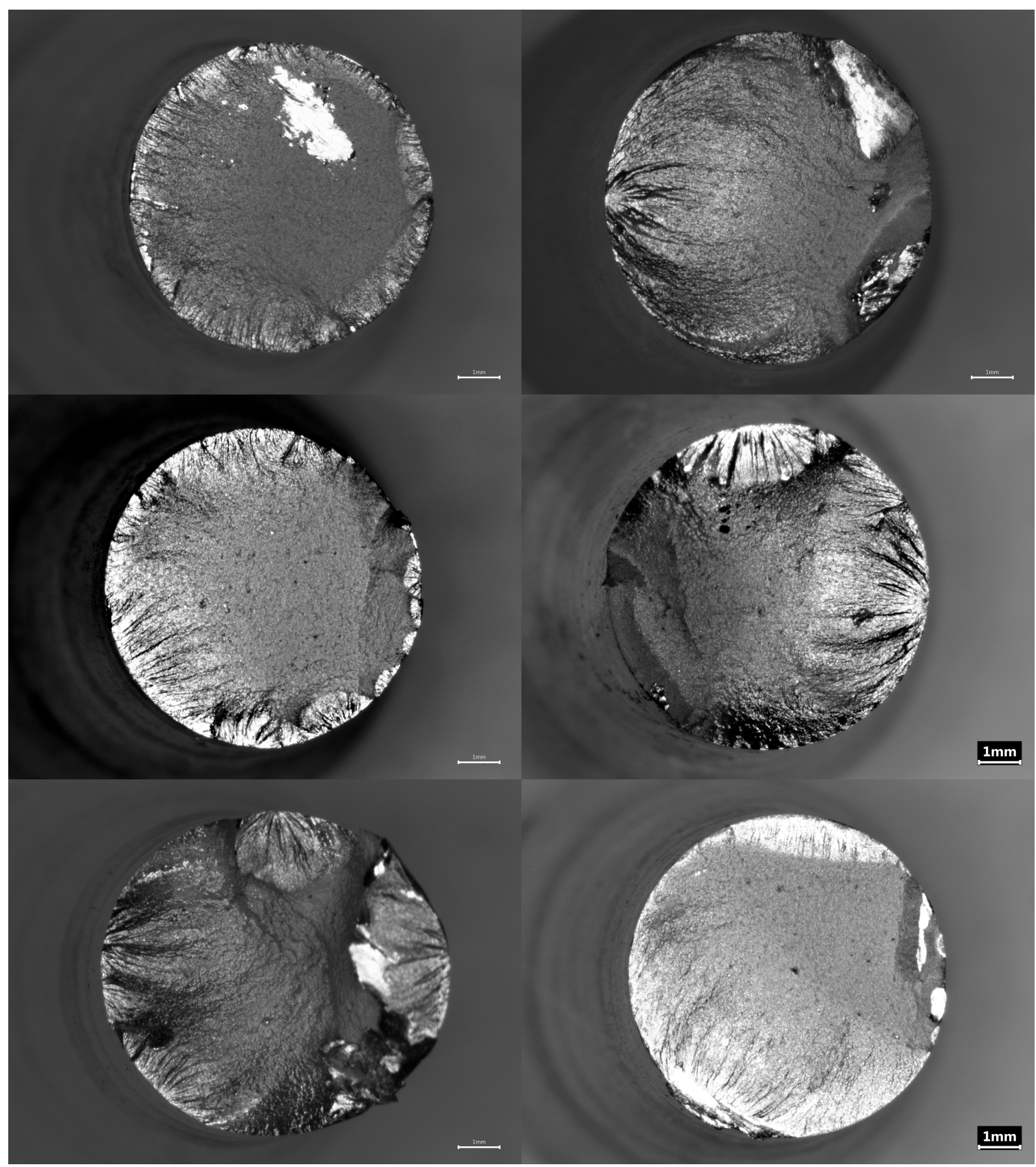

Figure 4.6: Left three specimens are subjected to 75\% UTS. Right three specimens are subjected to $55 \%$ UTS. $1 \mathrm{~mm}$ scale bar. 


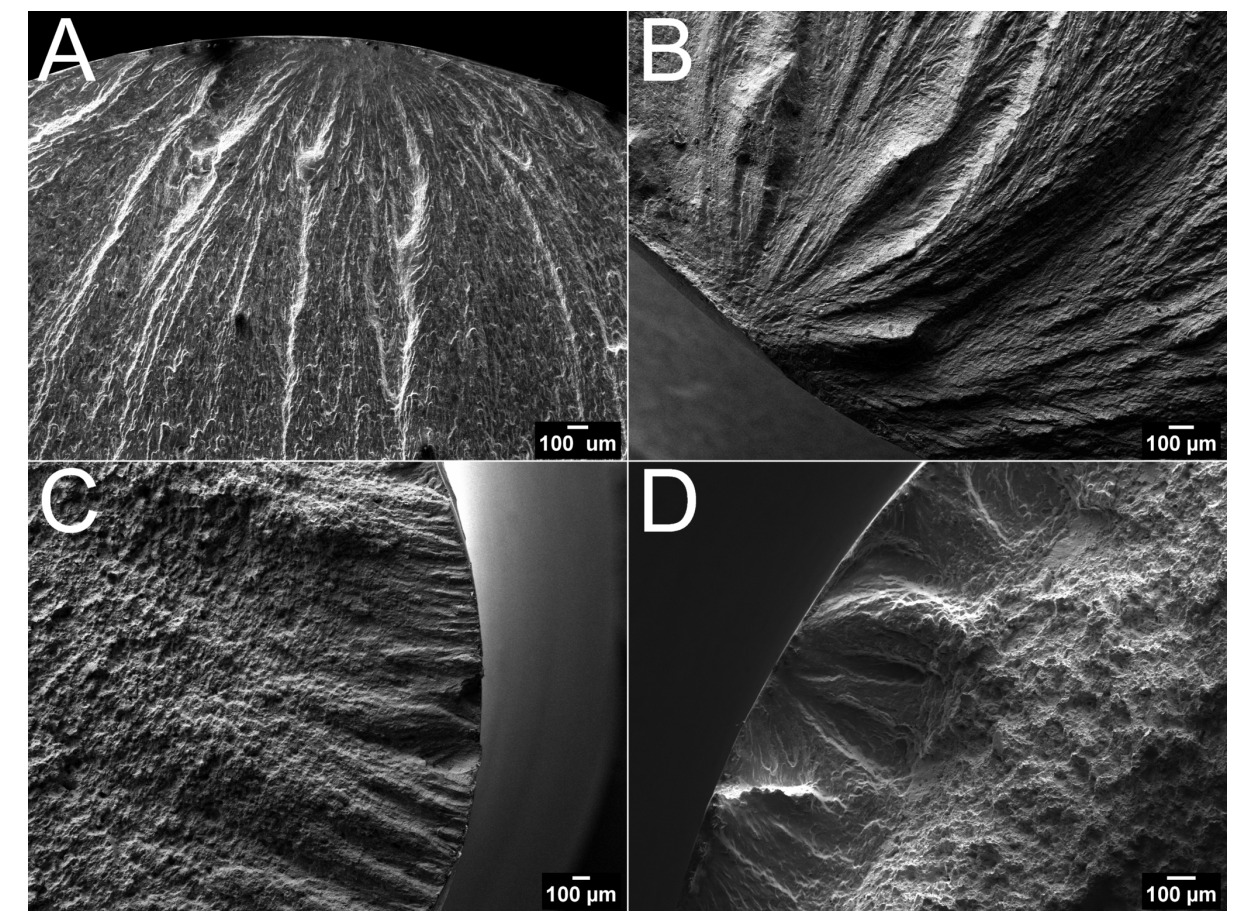

Figure 4.7: SEM photographs of specimens subjected to $44 \%$ UTS (A); $55 \%$ UTS (B); $75 \%$ UTS (C); and $86 \%$ UTS (D). 


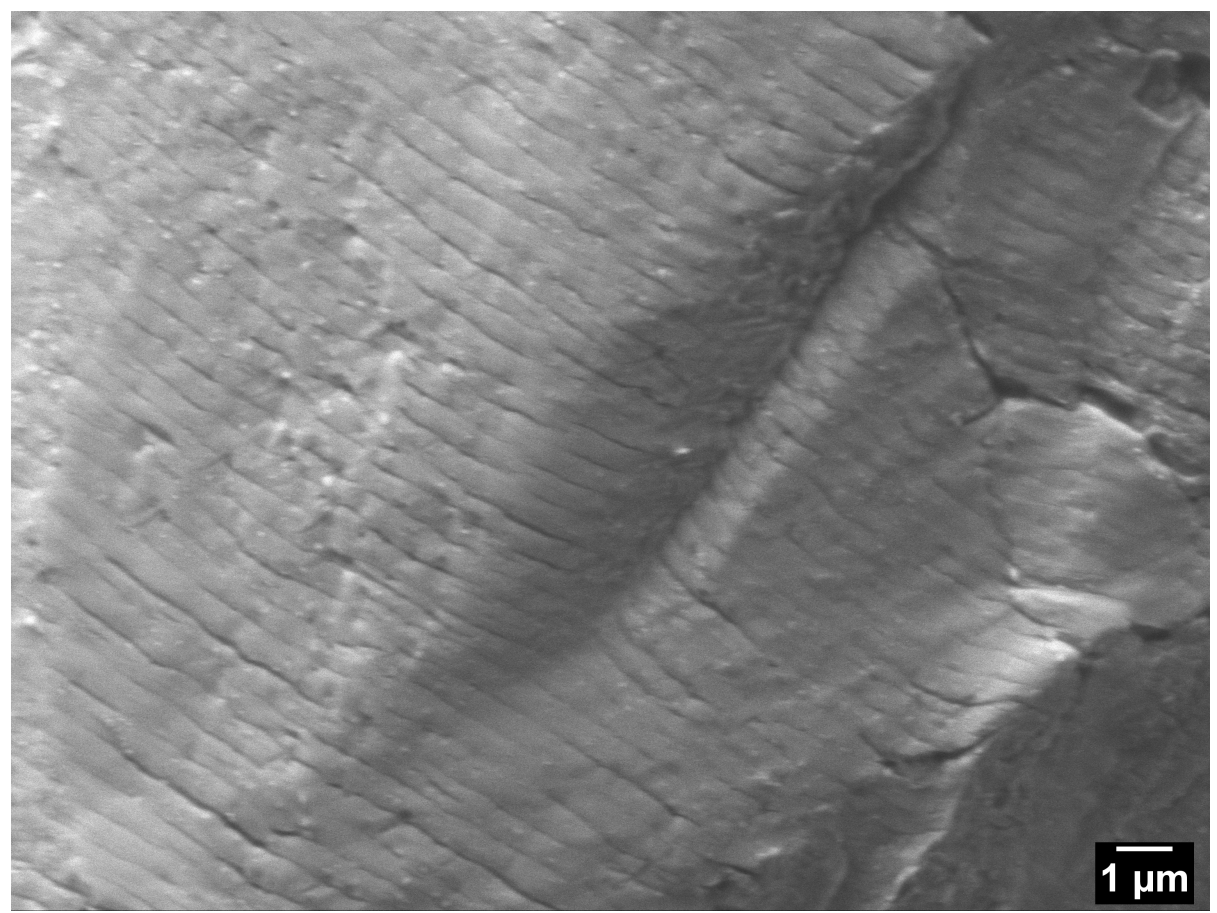

Figure 4.8: SEM photographs of striations for ECO7175v1-T74 subjected to $75 \%$ UTS. The striations, on average, have a width of approximately $0.481 \mu \mathrm{m}$. 


\section{Chapter 5}

\section{ECO7175v3-T74}

Table 5.1 shows the allocation of specimens used for ECO7175v 3 to maintain the desired $\approx 5 \%$ coefficient of variation with a confidence of $90 \%$ for the fatigue testing. Its composition is given in Table 5.2 and its tensile properties were given previously in Table 4.3.

Table 5.1: Allocation of Specimens for ECO7175v3-T74.

\begin{tabular}{cc}
\hline Stress Amplitude (MPa) & Number of Specimens \\
\hline 484 (88\% UTS) & 10 \\
424 (77\% UTS) & 10 \\
369 (67\% UTS) & 10 \\
$314(57 \%$ UTS $)$ & 10 \\
$259(47 \%$ UTS $)$ & 10 \\
$226(41 \%$ UTS $)$ & 6 \\
198 (36\% UTS) & 2 \\
$143(26 \%$ UTS $)$ & 2 \\
\hline
\end{tabular}

The experimental fatigue test results are tabulated in Table 5.3. Fatigue test results for stress amplitudes of $484 \mathrm{MPa}, 259 \mathrm{MPa}$, and $226 \mathrm{MPa}$ have a coefficient of variation of more than the desired 5\%, but are still within acceptable values. Fig. 5.1 show the S-N curve for ECO7175v3. The majority of cycles to failure above $200 \mathrm{MPa}$ are between $10^{3}$ and $10^{7}$ cycles. The fatigue strength of the material at $5 \times 10^{7}$ cycles is $220 \mathrm{MPa}$, approximately $40 \%$ of its tensile strength of $550 \mathrm{MPa}$. 
Table 5.2: Manufactured Composition of Aluminum ECO7175v3-T74.

\begin{tabular}{cc}
\hline Element & Composition \% \\
\hline Aluminum $(\mathrm{Al})$ & Balance \\
Calcium $(\mathrm{Ca})$ & 0.031 \\
Chromium $(\mathrm{Cr})$ & 0.002 \\
Copper $(\mathrm{Cu})$ & 1.28 \\
Iron $(\mathrm{Fe})$ & 0.07 \\
Magnesium $(\mathrm{Mg})$ & 2.42 \\
Manganese $(\mathrm{Mn})$ & 0.01 \\
Silicon $(\mathrm{Si})$ & 0.05 \\
Titanium $(\mathrm{Ti})$ & 0.045 \\
Zinc $(\mathrm{Zn})$ & 5.55 \\
\hline
\end{tabular}

In addition, fatigue failure scatter are generally low at $369 \mathrm{MPa}$ and higher, which corresponds to $67 \%$ and higher of its ultimate tensile strength (UTS), as shown in the small standard deviations. Below $369 \mathrm{MPa}$, fatigue failure scatter are higher, as shown in its larger standard deviations. ECO7175v1, in comparison, has a fatigue strength of approximately $207 \mathrm{MPa}$ at $10^{7}$ cycles, $36 \%$ of its tensile strength of 574 MPa [38]. Comparison of ECO7175v3 and ECO7175v1's S-N curves are shown in Fig. 5.2. 
Table 5.3: Statistical Analysis of Fatigue Testing for ECO7175v3-T74.

\begin{tabular}{c|ccc}
\hline Stress Amplitude (MPa) & Avg. Log Cycles & St.D. & Coeff. of Variation \% \\
\hline 484 (88\% UTS) & 3.799 & 0.111 & 2.9 \\
424 (77\% UTS) & 4.170 & 0.107 & 2.6 \\
369 (67\% UTS) & 4.473 & 0.101 & 2.3 \\
314 (57\% UTS) & 5.033 & 0.214 & 4.2 \\
259 (47\% UTS) & 5.577 & 0.348 & 6.2 \\
226 (41\% UTS) & 5.720 & 0.363 & 6.3 \\
198 (36\% UTS) & \multicolumn{2}{|c}{ Run-out (Over $5 \times 10^{7}$ Cycles) } \\
143 (26\% UTS) & \multicolumn{2}{c}{ Run-out (Over $5 \times 10^{7}$ Cycles) } \\
\hline
\end{tabular}

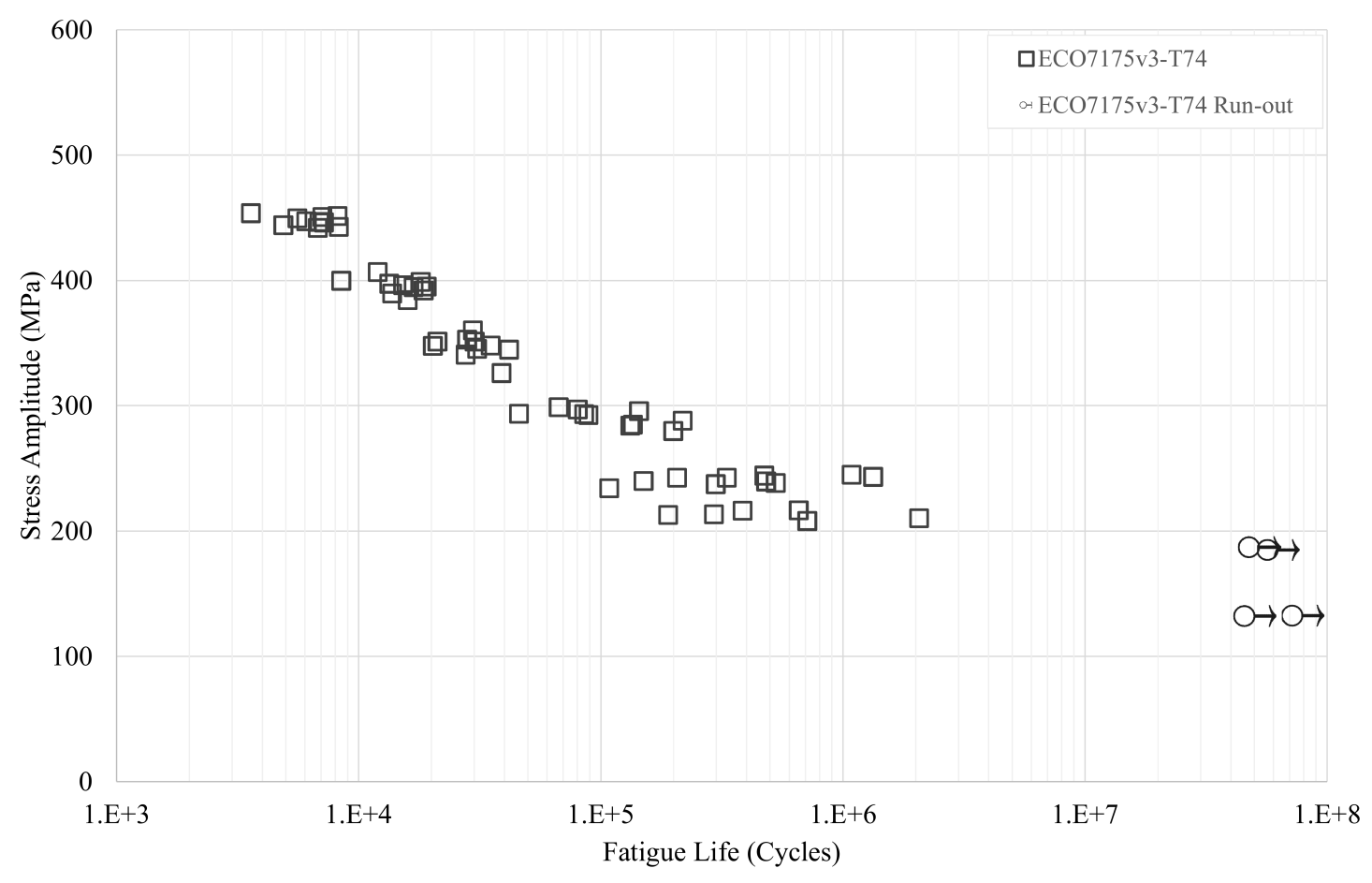

Figure 5.1: S-N data plot of aluminum ECO7175v3-T74. At higher stress levels above $55 \%$ UTS, the scatter in the data are low. At stresses 55\% UTS and lower, the scatter in the data are high. 


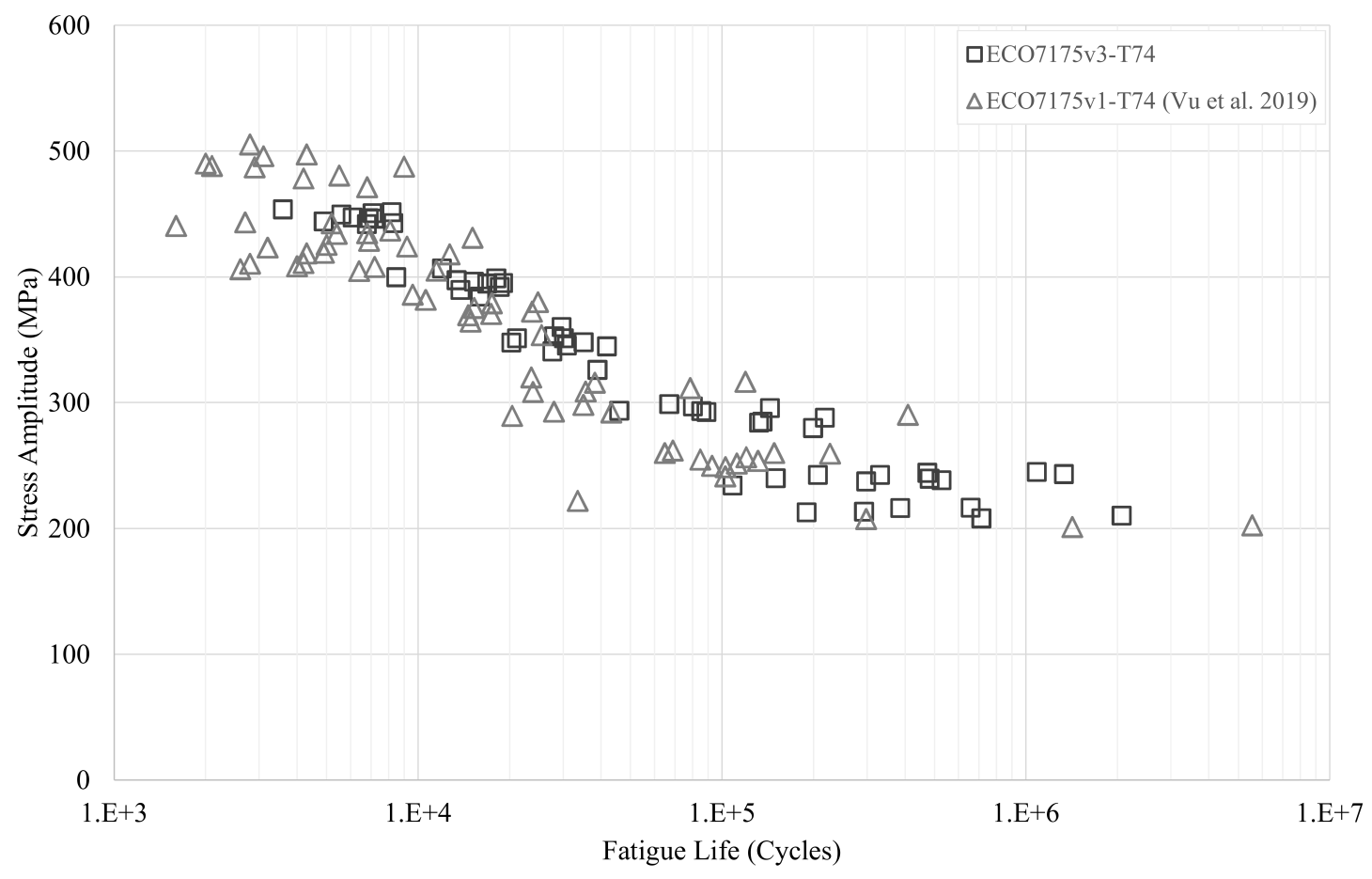

Figure 5.2: S-N data plot of aluminum ECO7175v3-T74 vs ECO7175v1-T74 [38]. Compared to ECO7175v1-T74, the scatter for ECO7175v3-T74 is generally lower.

\subsection{Basquin's Equation}

The fatigue life data was fitted to Basquin's equation to determine its constants (Fig. 5.3). The fatigue strength coefficient, $\sigma_{F}^{\prime}$, was determined to be $1589.7 \mathrm{MPa}$ and the fatigue strength exponent, $b$, was determined to be -0.145. As the fatigue strength coefficient, $\sigma_{F}^{\prime}$, is approximately equal to the material's fracture strength [15], $\sigma_{F}$, the result indicates ECO7175v3 required considerably higher stresses to cause a fatigue failure in one stress reversal compared to ECO7175v1. 


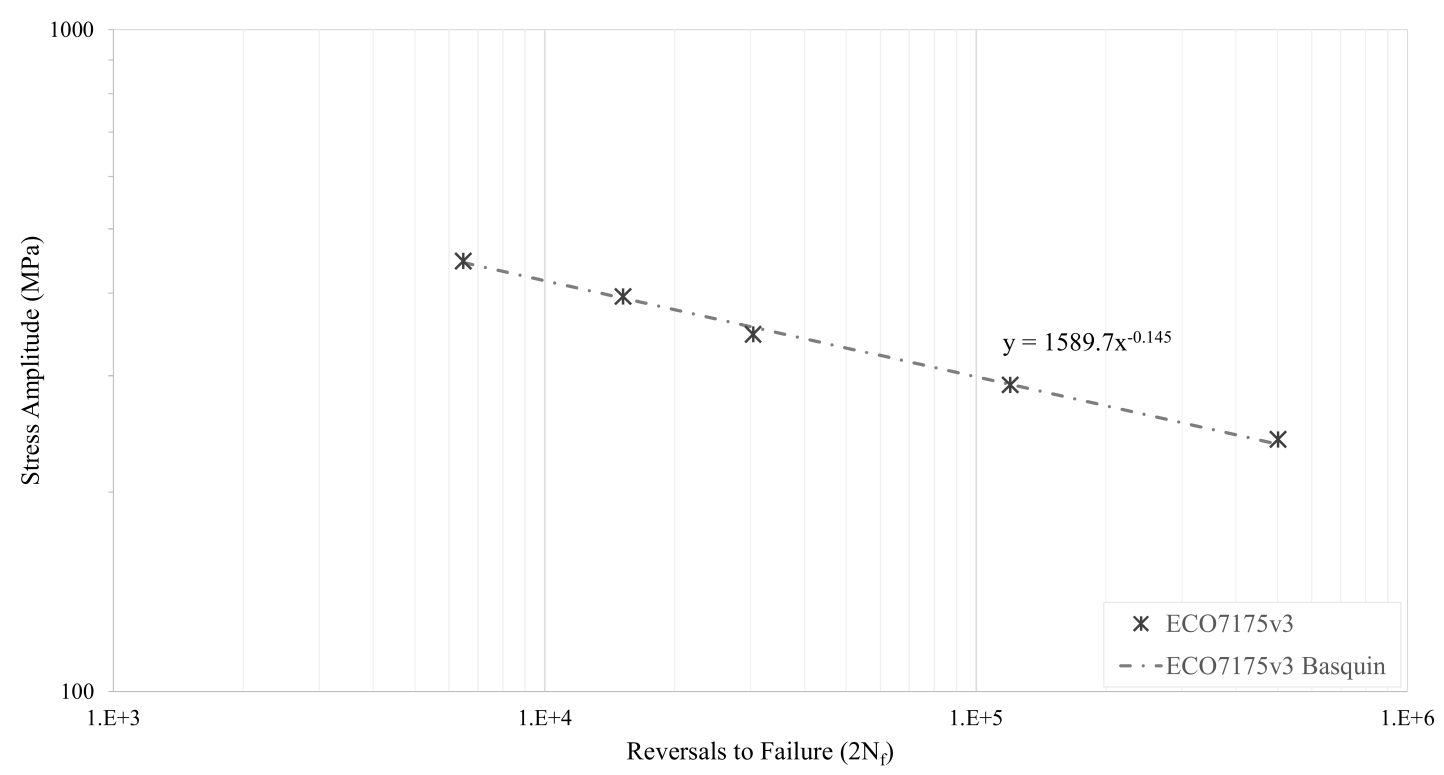

Figure 5.3: Fatigue data for ECO7175v3-T74 curve-fitted to Basquin's equation to determine the material's fatigue strength coefficient, $\sigma_{F}^{\prime}$, and fatigue strength exponent, $b$.

\subsection{Fracture Analysis}

Fracture surfaces between ECO7175v3 specimens at various stress levels are compared. Fractography results show specimens subjected to stresses of $77 \%$ or higher of its tensile strength, as shown in Fig. 5.4, have fracture features that are sharply defined. Fractography also shows clear crack initiation points and failure modes that are predominately ductile at the surface while brittle towards the center. The fracture characteristics of ECO7175v3 at 77\% UTS and higher are comparable to those of ECO7175v1 at 86\% UTS and higher. However, fractography results for ECO7175v3 specimens subjected to stresses of $67 \%$ and lower of its tensile strength, as shown in Fig. 5.5, have softly defined fracture features and propagation bands that are hard 
to discern with an unaided eye. Failure modes are generally ductile throughout the entire fracture surface. SEM imaging results of these predominately ductile surfaces are shown in Fig. 5.6. These fracture characteristics for ECO7175v3 at $67 \%$ UTS and lower are unique as they were not observed in ECO7175v1. Comparisons between fracture surfaces of ECO7175v3 and ECO7175v1 are shown in Fig. 5.8.

Fig. 5.10 compares the fracture surfaces of ECO7175v3 and ECO7175v1 at similar magnification under the SEM. It can be seen that for both surfaces, the fractures are intergranular, meaning the cracks propagate through the grain boundaries. However, ECO7175v3 has considerably larger crack widths and larger changes in its topography. This is attributed to differences in grain sizes that was later determined in metallography. The larger grain sizes may also be what gives the fracture surfaces of ECO7175v3 a shiny look. Fig. 5.11 shows another SEM comparison for a different set of specimens with similar characteristics.

Similarly to ECO7175v1, irrespective of the stresses the specimens are subjected to, all crack initiation points for ECO7175v3 are located at the surface and no inclusions to act as stress concentrators are seen, as shown under SEM imaging in Fig. 5.9 .

Striations were found and measured. The striations, on average, have a width of approximately $1.164 \mu \mathrm{m}$, as shown in Fig. 5.12. The striations are considerably larger than ECO7175v1 of approximately $0.481 \mu \mathrm{m}$. This indicates for each cycle of fatigue load, ECO7175v3 fractures more compared to ECO7175v1. 


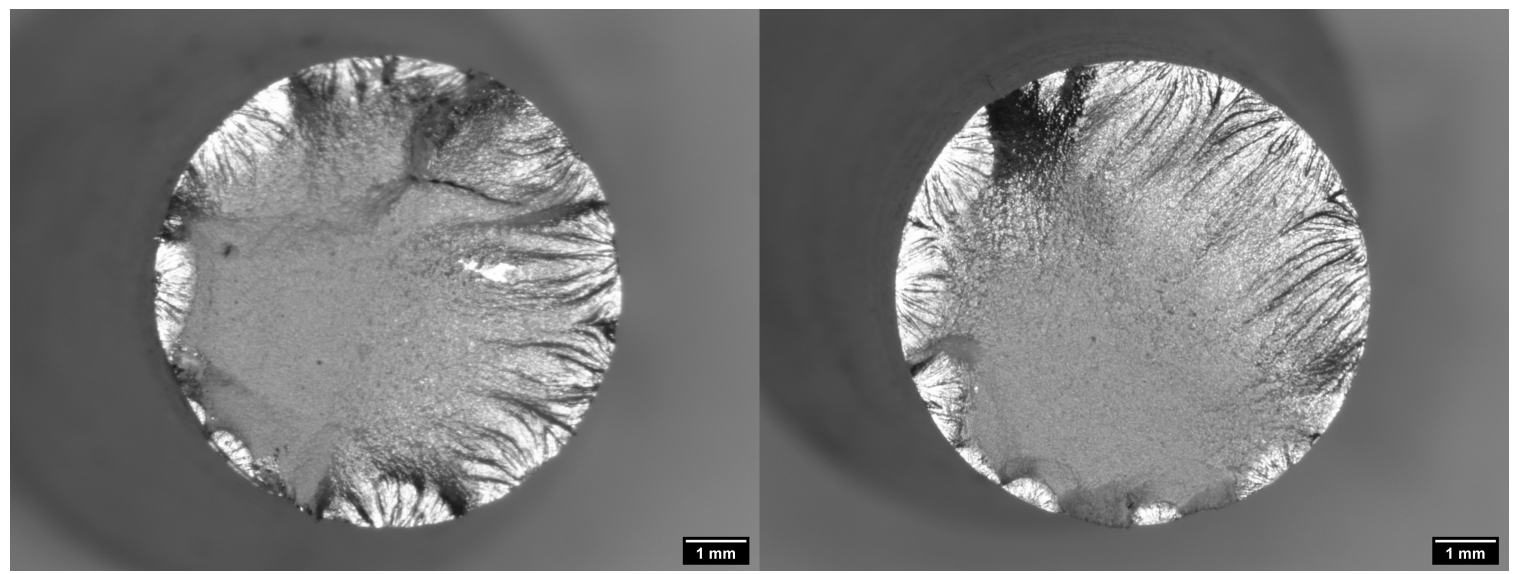

Figure 5.4: Specimens subjected to $88 \%$ UTS (left) and $77 \%$ UTS (right) has fracture features that are well defined with long beach marks, clear crack initiation sites, and has failure modes that are predominately ductile near the surface at fracture initiation points while brittle towards the center closer to the final fracture rupture points. 


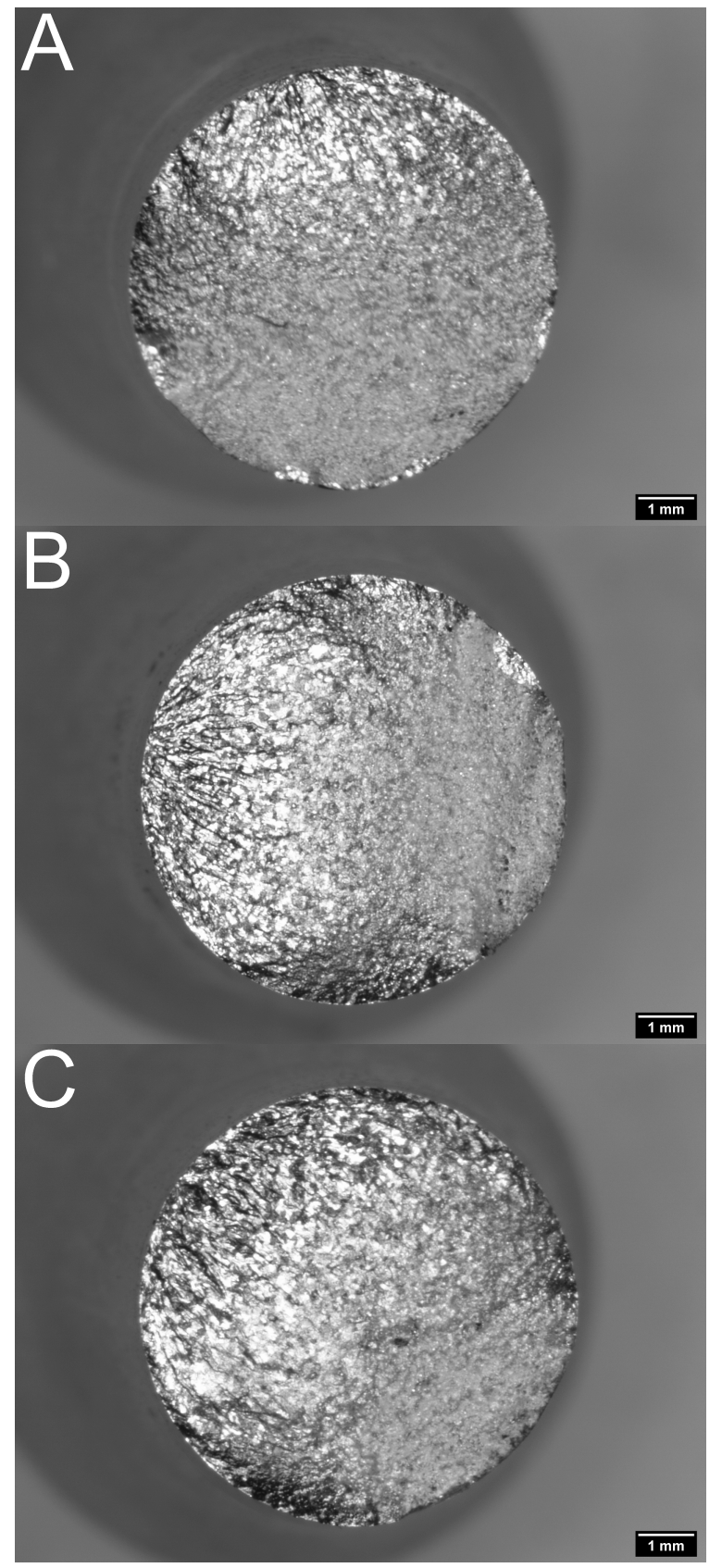

Figure 5.5: Specimens subjected to 67\% UTS (top), 57\% UTS (center), 47\% UTS (bottom), and below (not shown) has fracture features that are not well defined, have failure modes that are predominately ductile through the majority of the fracture surface, and are characteristically shiny. 


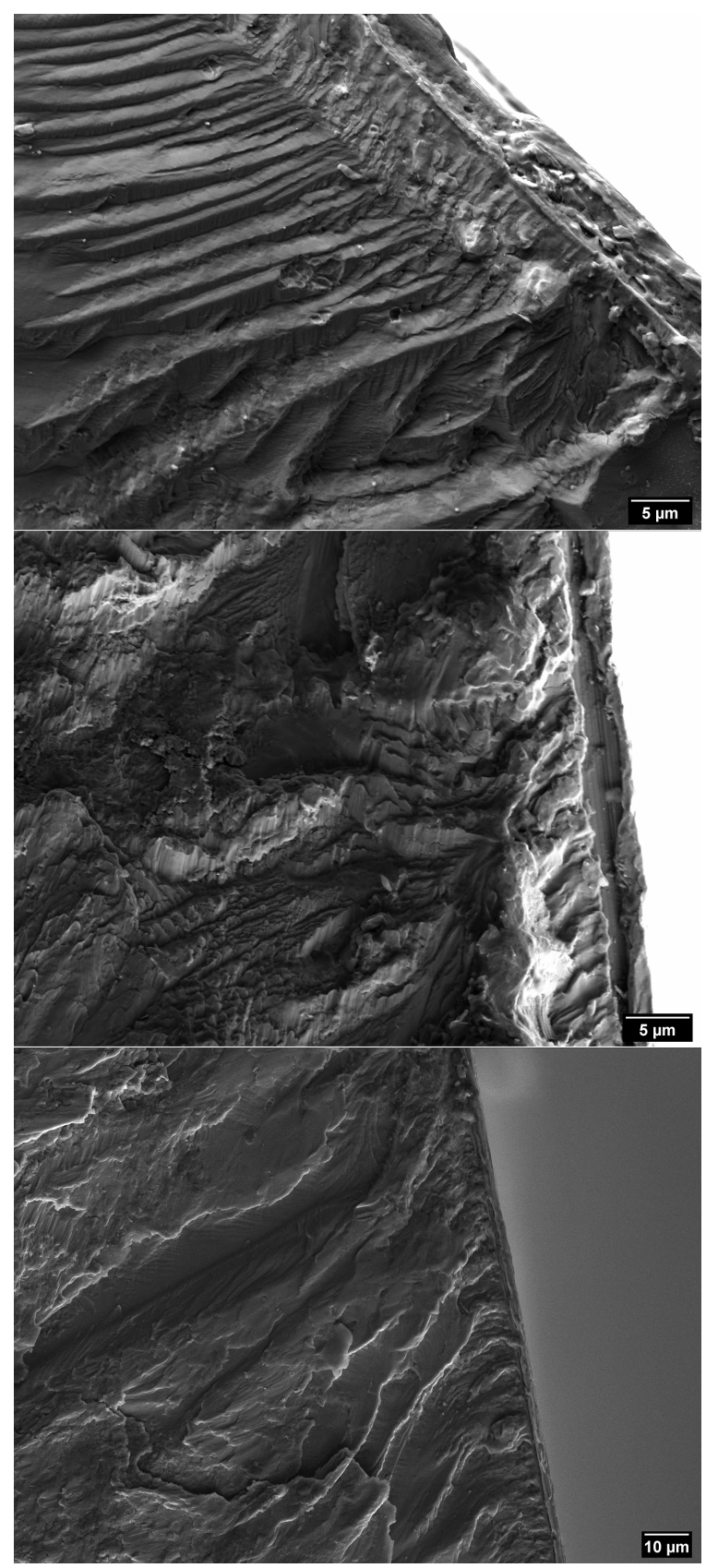

Figure 5.6: High power SEM images of ECO7175v3-T74 at various crack initiation points with no clear indication of inclusions to act as the stress concentrators. 

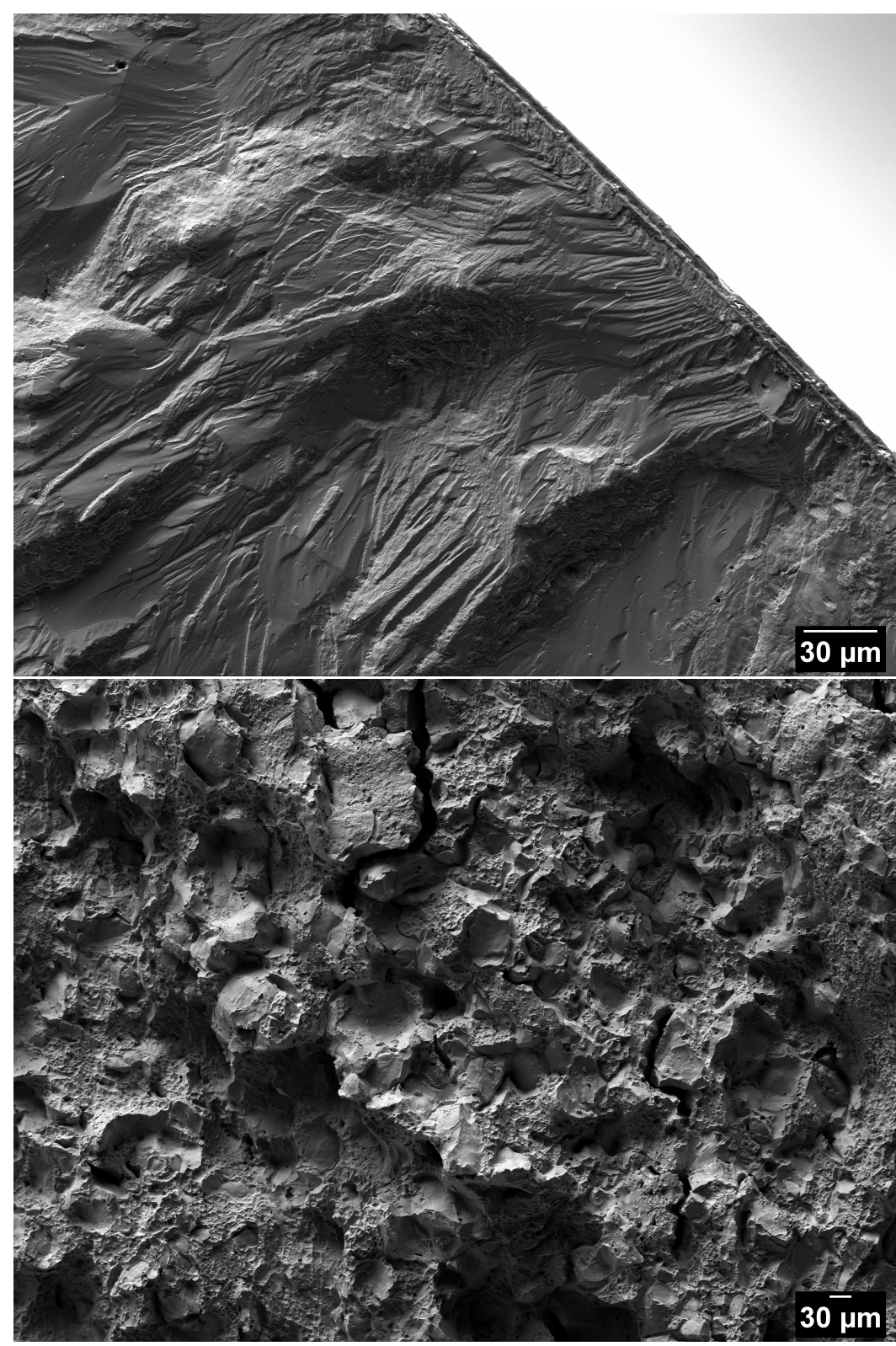

Figure 5.7: The top figure shows a closeup near ECO7175v3-T74's crack initiation point. The bottom figure shows ECO7175v3-T74's fracture surfaces at other parts of the surface away from the crack initiation points. At the initiation points, the fracture is very sharp and looks like facets of gems, indicating it is a transgranular fracture where fracture cuts through the grains. At other areas away from the initiation points, the fractures are intergranular, meaning the fractures travels through the grain boundaries. 


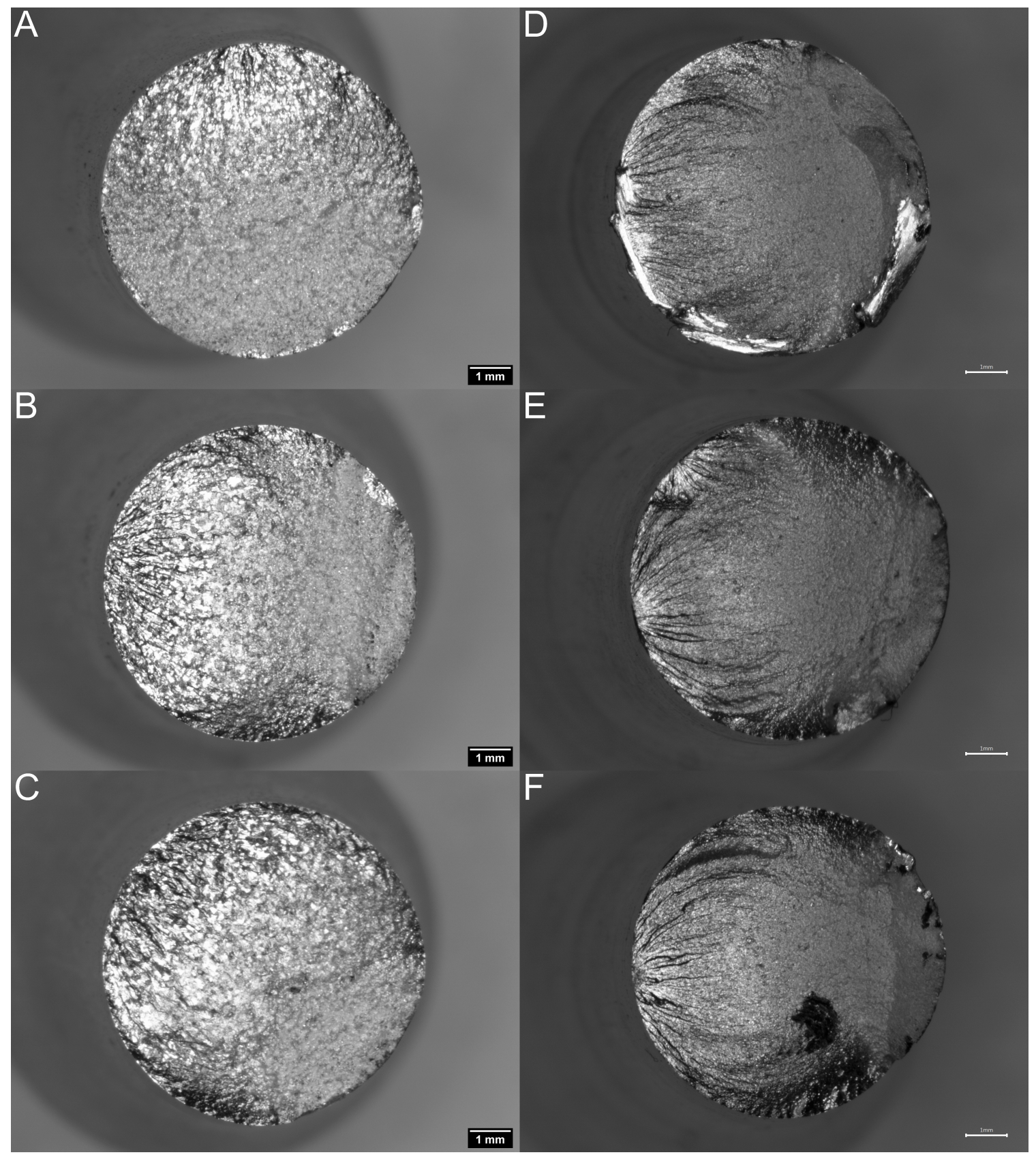

Figure 5.8: Left three are ECO7175v3-T74 specimens subjected to $67 \%$ UTS (A), $57 \%$ UTS (B), and 47\% UTS (C). Right three are ECO7175v1-T74 specimens subjected to similar stresses at $66 \%$ UTS (D), $55 \%$ UTS (E), and $44 \%$ UTS (F). 


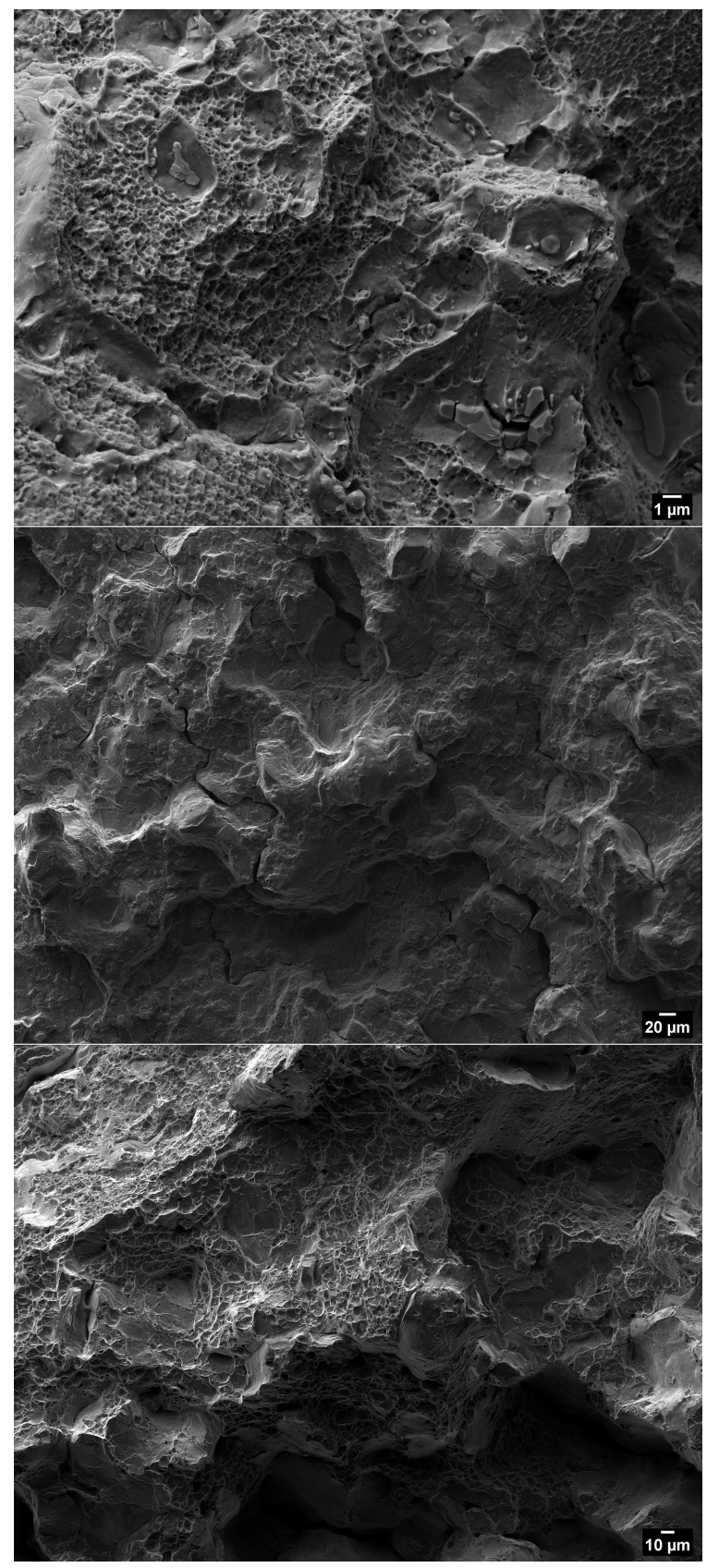

Figure 5.9: SEM images of ECO7175v3-T74 showing different surface characteristics across the fracture surface. In the top and bottom image, cone and cups can be ne noticed. These indicates the fracture at these points are ductile in nature. 


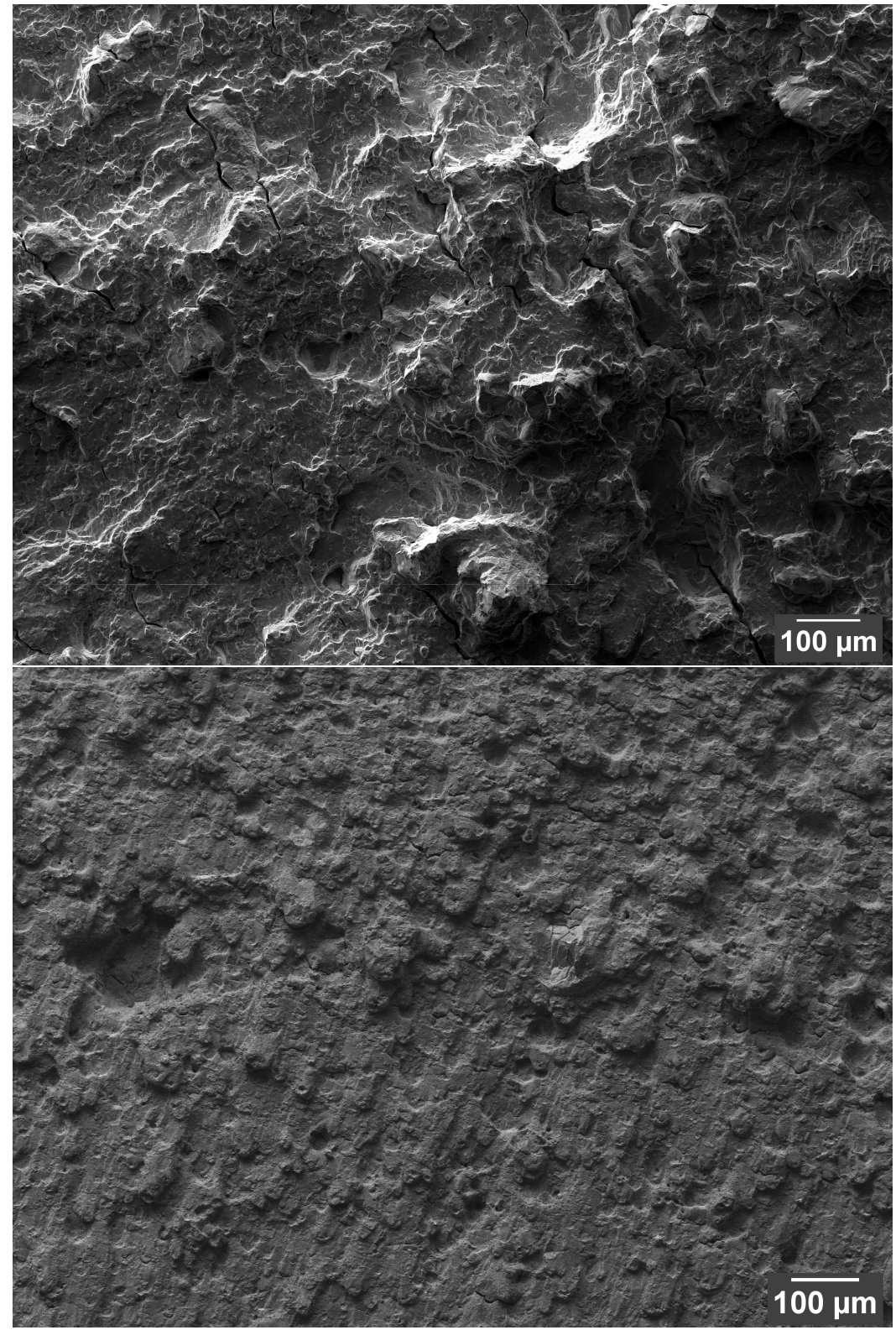

Figure 5.10: The top figure shows the a closeup of ECO7175v3-T74's surface. The bottom figure shows ECO7175v1-T74 at a similar magnification. Both surfaces have intergranular fracture characteristics but ECO7175v3-T74 has larger fractures. This is be caused by larger grain sizes in ECO7175v3. The larger grain sizes and the resulting fracture characteristics is what gives the ECO7175v3-T74's fracture surface a shiny look. 


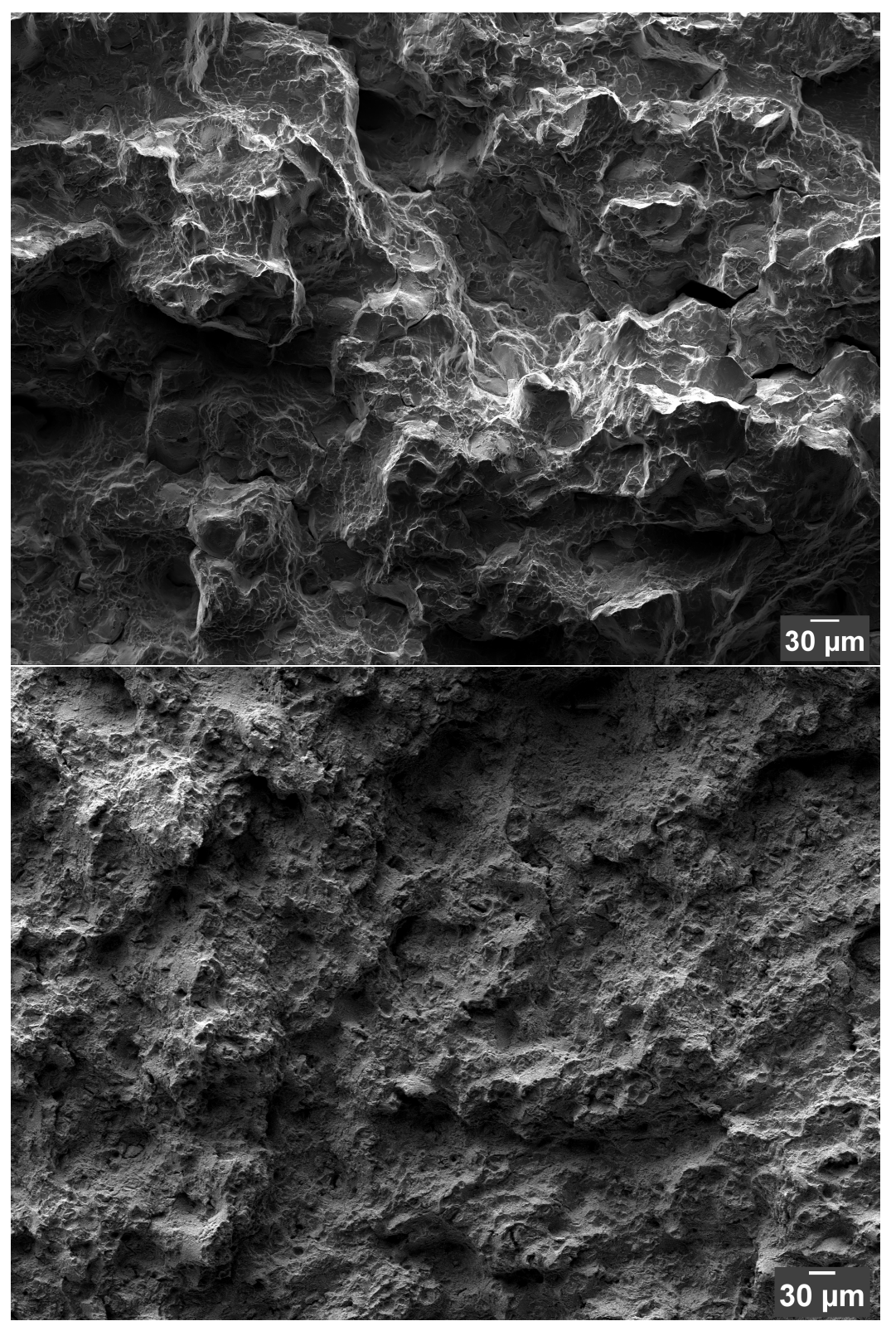

Figure 5.11: The top figure shows the a closeup of ECO7175v3-T74. The bottom figure shows ECO7175v1-T74 at a similar magnification. The larger grain sizes in ECO7175v3-T74 is what gives it its characteristic shiny fracture surface. 


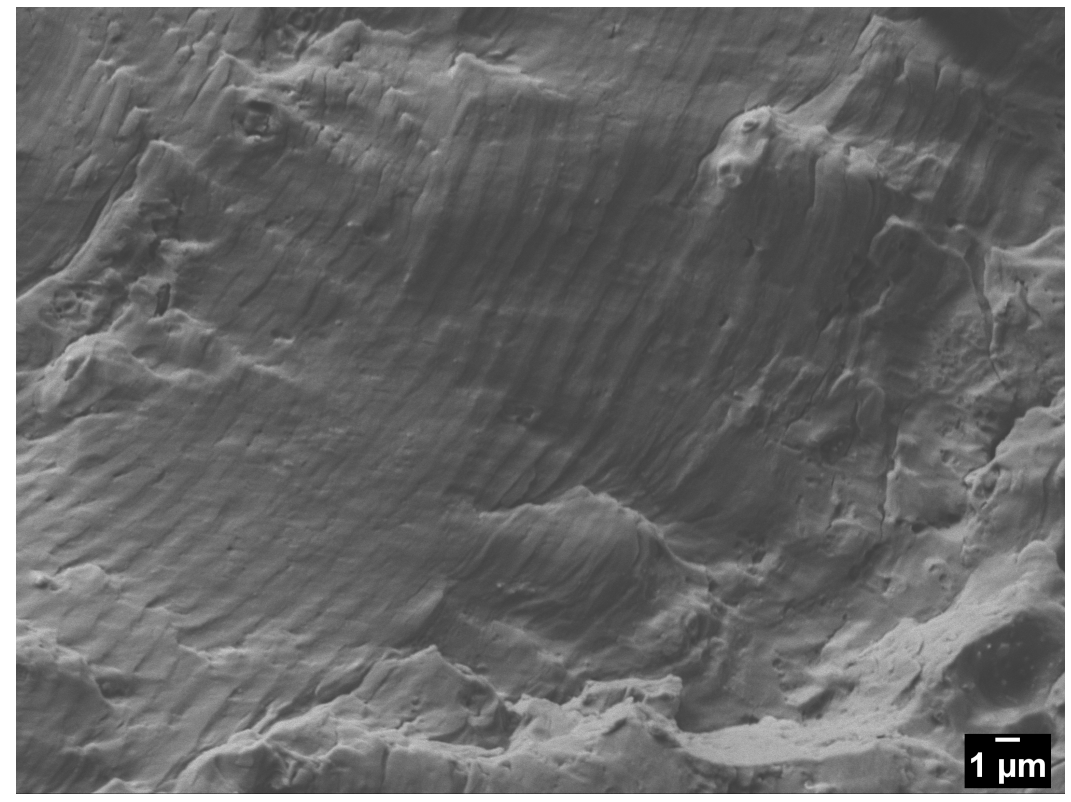

Figure 5.12: SEM photographs of striations for ECO7175v3-T74 subjected to $77 \%$ UTS. The striations, on average, have a width of approximately $1.164 \mu \mathrm{m}$. 


\subsection{Metallography}

Grain sizes of ECO7175v3, shown in Fig. 5.13, were measured. On average, the grain sizes of ECO7175v3 are approximately $78 \mu \mathrm{m}$. The grain sizes are significantly larger than the average grain sizes of $10 \mu \mathrm{m}$ for ECO7175v1 (Fig. 5.14) and ECO7175v2. This is attributed to the lack of chromium in ECO7175v3. Chromium can be used to prevent recrystallization in aluminum-magnesium-zinc alloys [43]. As a result, ECO7175v1 and ECO7175v2's recrystallization were suppressed. Therefore, ECO7175v1 and ECO7175v2 maintained their grain sizes and geometry.

During early grain analysis, many irregular shaped black and gray particles on the surfaces of the ECO7175v3 specimens were noticed. These particles were also noticed on ECO7175v1 specimens. Differential interference contrast microscopy (DIC), also known as Nomarski interference contrast (NIC), or simply Nomarski microscopy, was then used to acquire depth information. These black and gray particles are revealed to be precipitates. They are recognized as precipitates as they are part of the matrix rather than simply sitting on the surface of the material as surface contaminants. Fig. 5.15 and Fig. 5.16 shows the surfaces of an ECO7175v3 and ECO7175v1 specimen under Nomarski.

The number of particles was compared between ECO7175v3 and ECO7175v1. ECO7175v3 has higher quantities of particles compared to ECO7175v1. ECO7175v1, however, has slightly higher quantities of larger particles. The polished surfaces of 

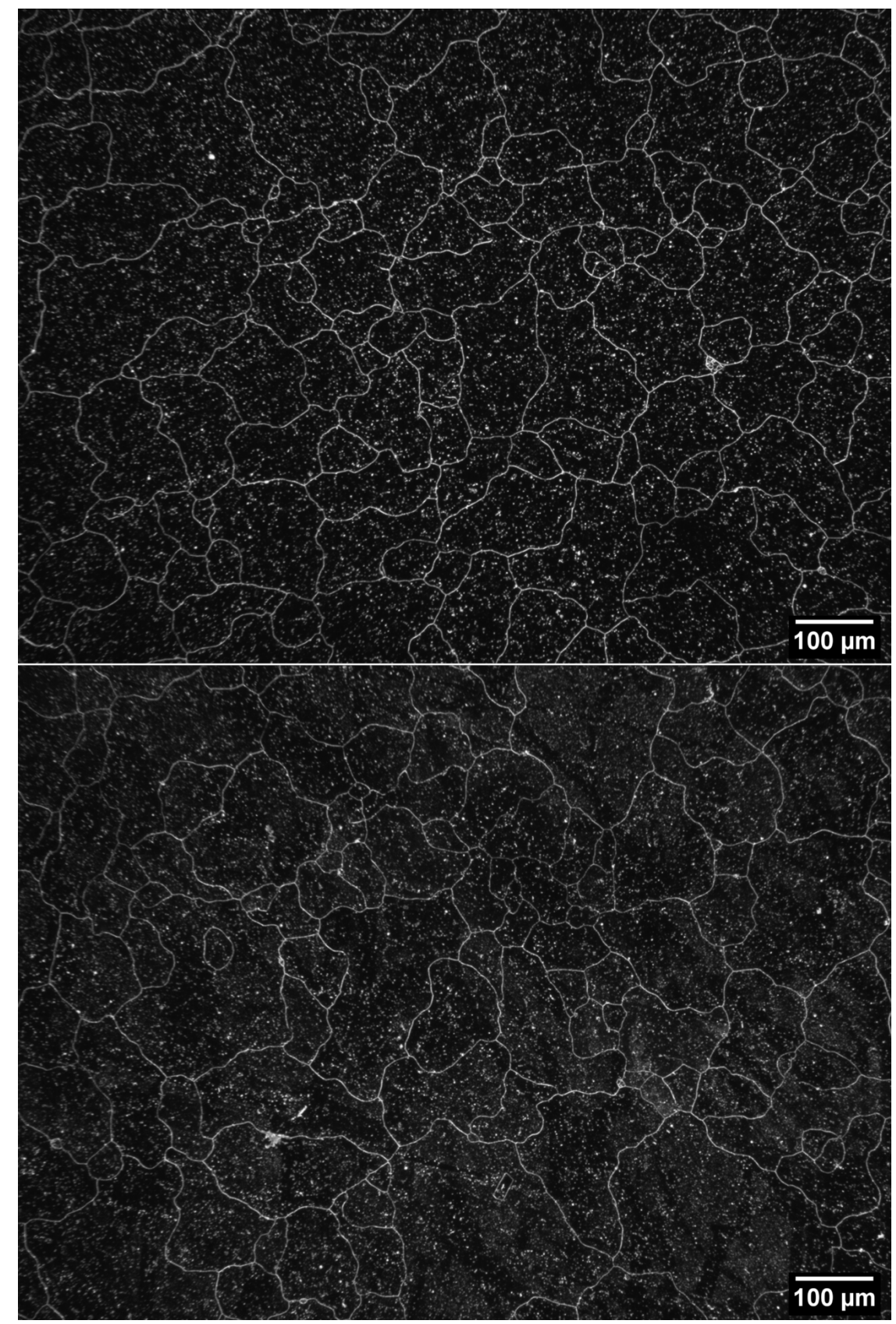

Figure 5.13: Metallographs of ECO7175v3-T74 specimens showing the grains and grain boundaries. The average grain size is $78 \mu \mathrm{m}$. The grain sizes are considerably larger than the average grain size of $710 \mu \mathrm{m}$ for the other two variants. This is contributed by the very low chromium content, which is used to prevent recrystallization in aluminum-magnesium-zinc alloy [43]. 

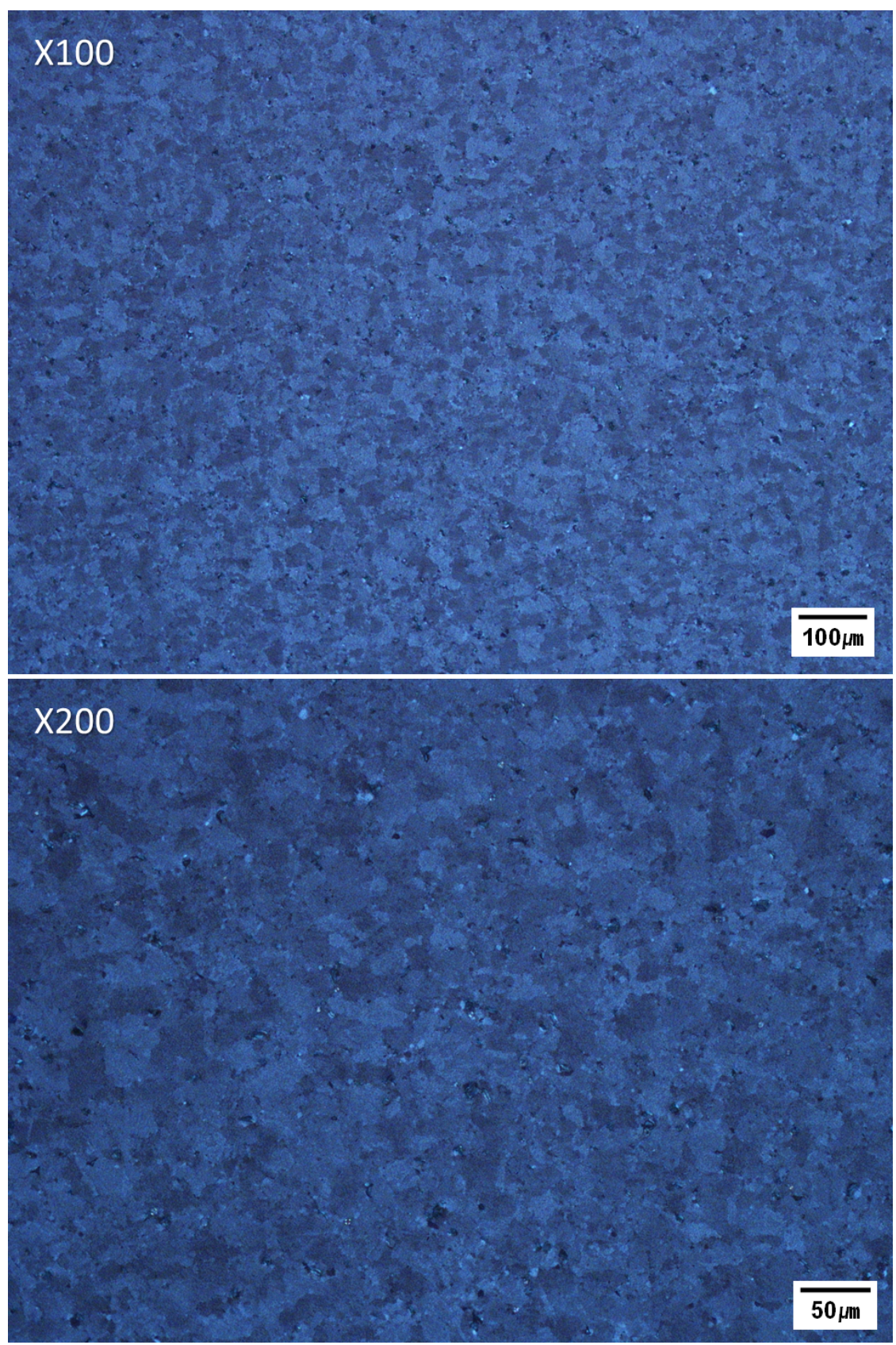

Figure 5.14: Metallographs of ECO7175v1-T74 specimens showing the grains and grain boundaries.

ECO7175v3 under dark field imaging to highlight the particles are shown in Fig.

5.17. Using image processing, the number of particles and their sizes between the two 


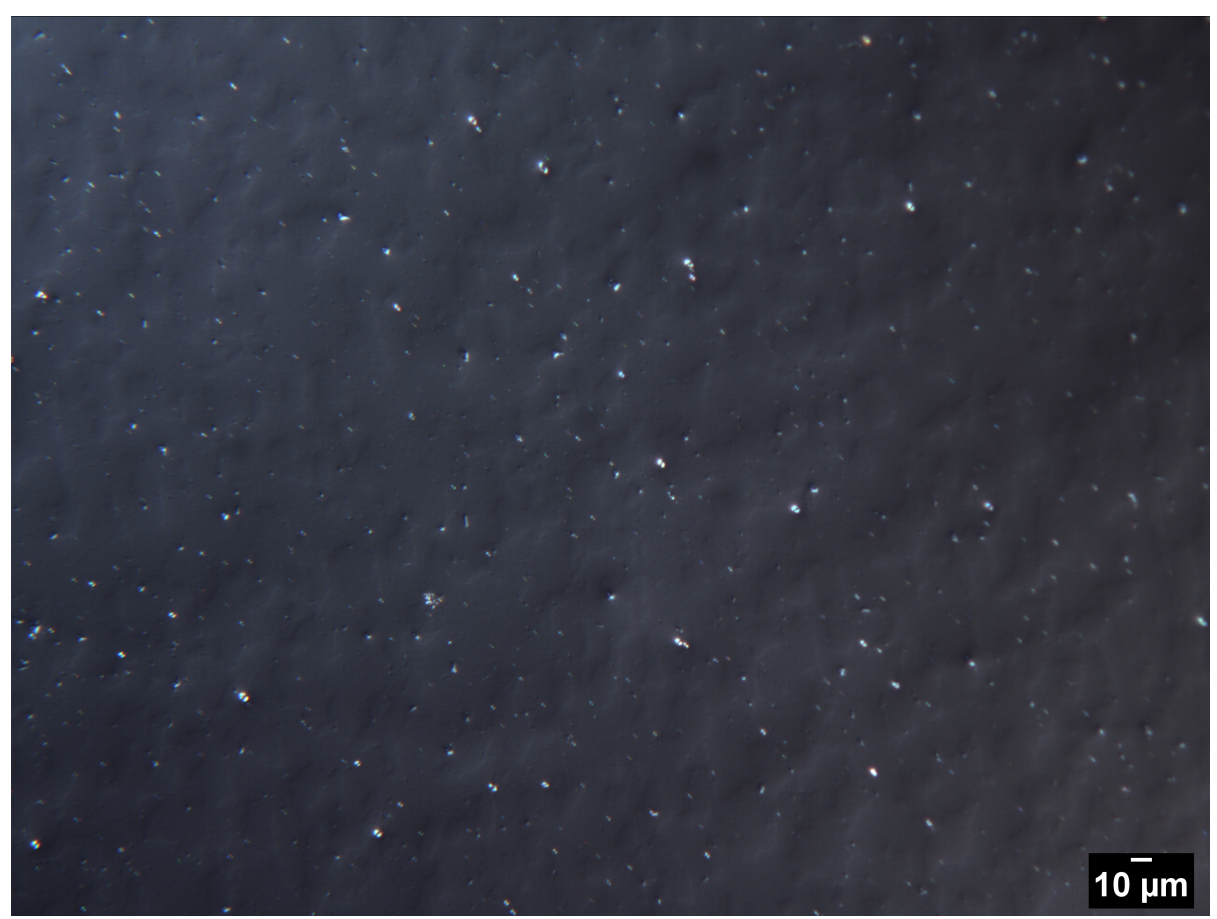

Figure 5.15: ECO7175v3-T74 polished surface under Nomarski exposed many irregular shaped bumps on the surface of the specimen. These bumps are recognized as precipitates as they are part of the matrix rather than surface contaminants. At 200x magnification.

variants were counted and are shown in Fig. 5.19 and Fig. 5.20.

The particles in ECO7175v3 were measured to have a volume fraction of approximately 2.5\%. ECO7175v1's volume fraction was calculated to be approximately 1.6\%. The use of Energy-dispersive X-ray spectroscopy (EDX) shows the precipitates in ECO7175v3 generally have high concentrations of $\mathrm{Fe}, \mathrm{Cu}, \mathrm{C}, \mathrm{Zn}, \mathrm{Mg}$, and $\mathrm{Ca}$. The EDX analysis results for ECO7175v3 are shown in Fig. 5.21 with its associated Table 5.4. In the case of ECO7175v1, the precipitates also have high concentrations of Fe, $\mathrm{Cu}, \mathrm{C}, \mathrm{Zn}, \mathrm{Mg}$, and $\mathrm{Ca}$, but $\mathrm{Cr}$ is also found in high concentration. 


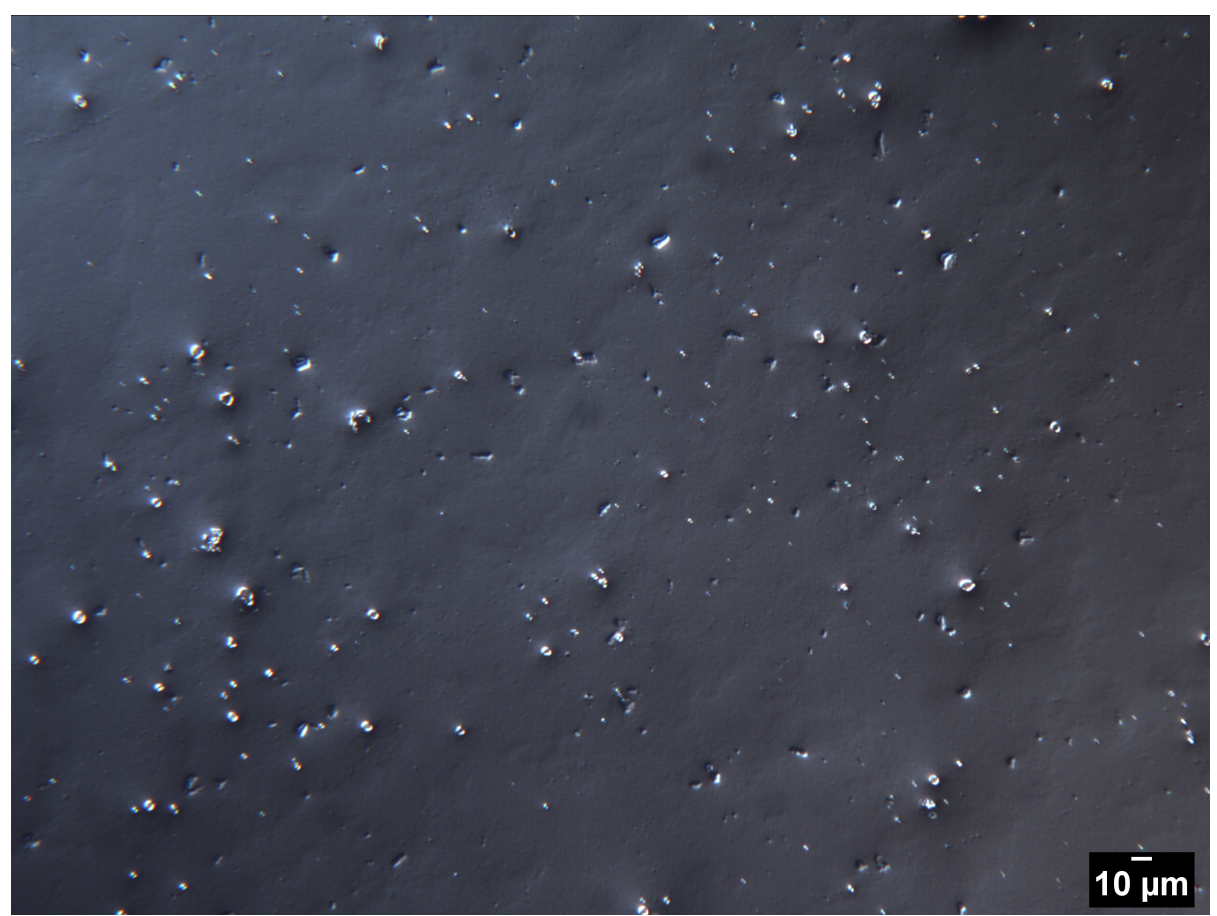

Figure 5.16: ECO7175v1-T74 polished surface under Nomarski. The precipitates are seemingly larger than precipitates on ECO7175v3-T74 specimens. At 200x magnification.

Table 5.4: EDX (Energy-dispersive X-ray spectroscopy) Spot Analysis for ECO7175v3-T74

\begin{tabular}{l|ccccc}
\hline Elements & Spot 1 & Spot 2 & Spot 3 & Spot 4 & Spot 5 \\
\hline Al (wt \%) & 58.9 & 58.8 & 76.2 & 81.2 & 84.9 \\
Fe (wt \%) & 22.8 & 1.3 & 0.4 & - & 1.5 \\
Cu (wt \%) & 8.0 & 19.9 & 9.4 & 3.6 & 2.1 \\
C (wt \%) & 3.9 & 4.0 & 2.9 & 3.3 & 3.6 \\
Zn (wt \%) & 3.1 & 9.6 & 7.2 & 6.1 & 5.5 \\
Mg (wt \%) & 1.0 & 1.5 & 1.9 & 4.1 & 1.9 \\
\hline
\end{tabular}




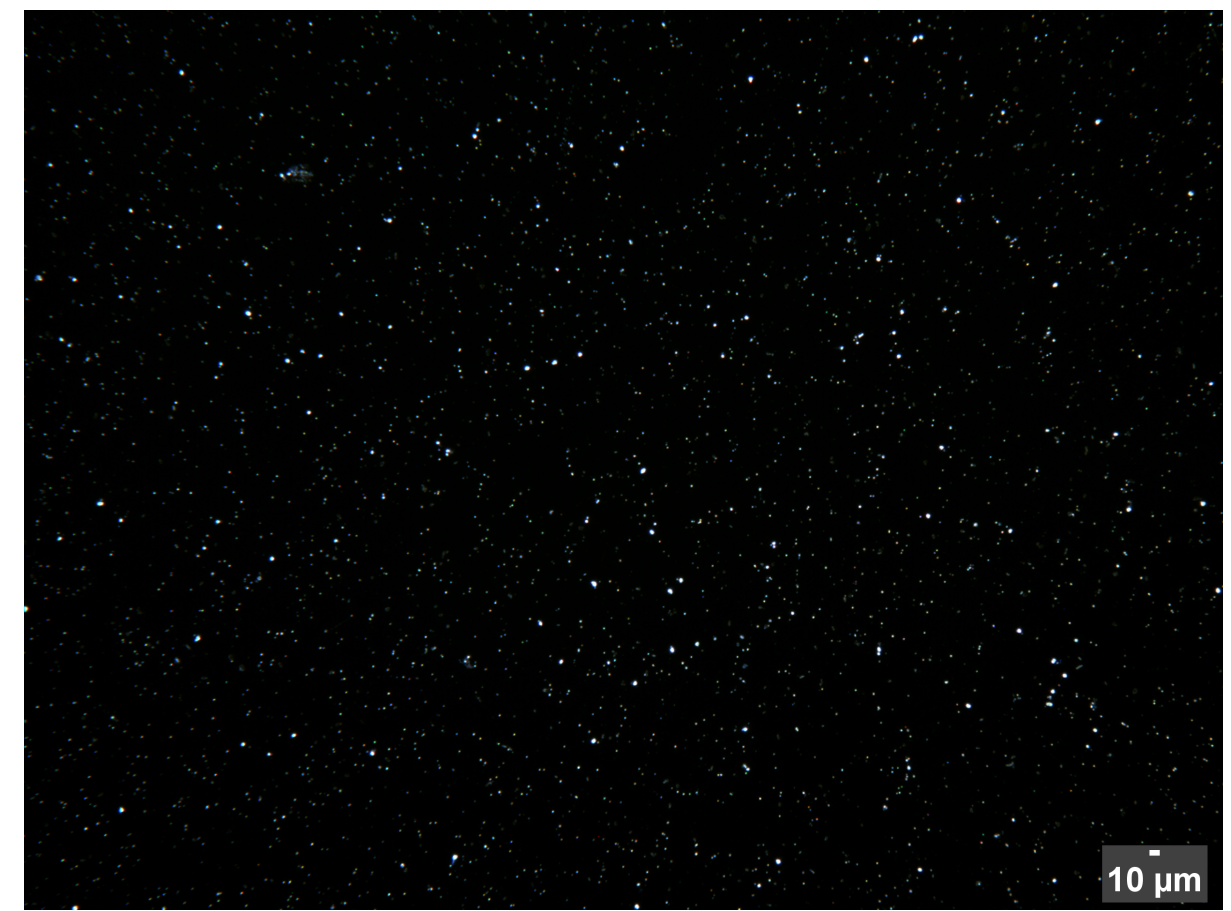

Figure 5.17: Metallograph of highly polished surface of ECO7175v3-T74 under dark field imaging to highlight particles on the surface. At 100x magnification. 


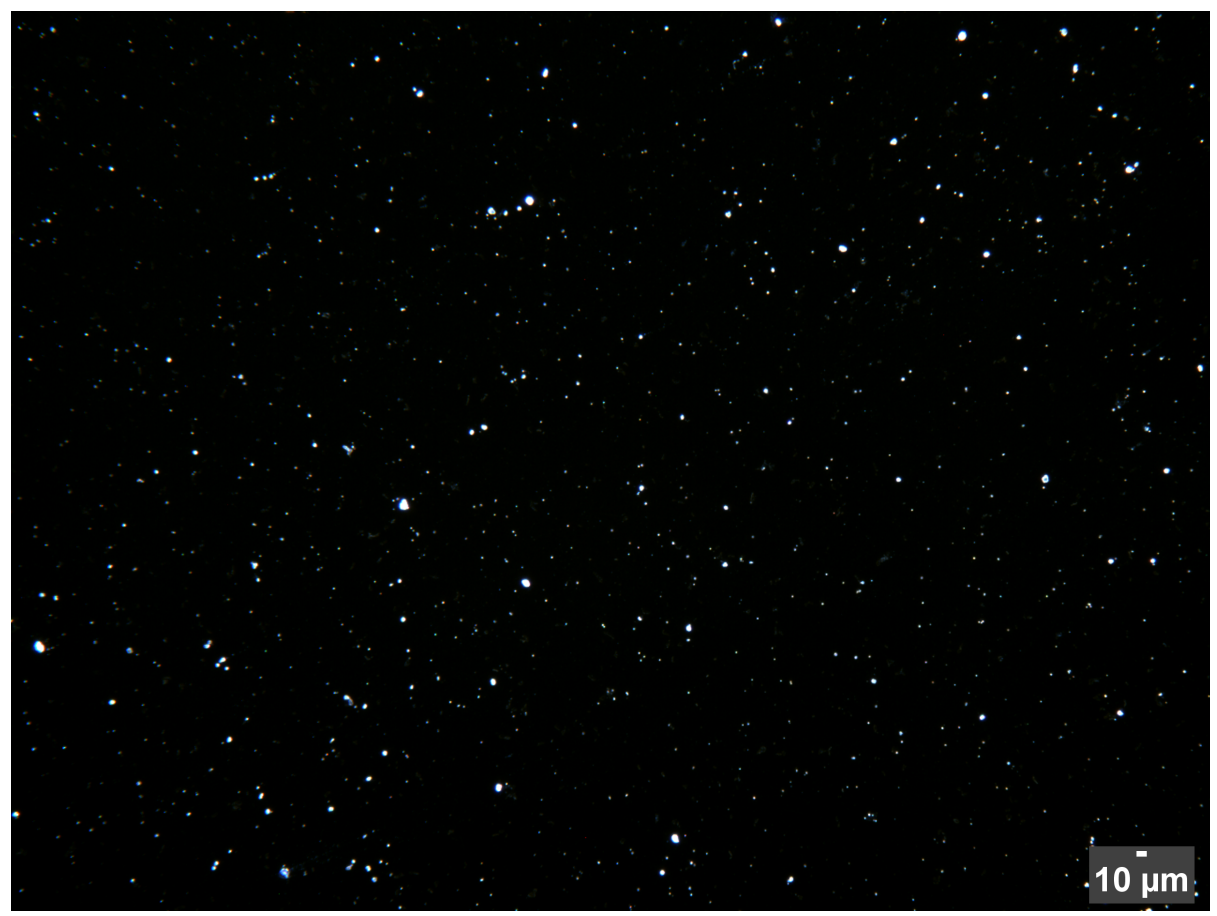

Figure 5.18: Metallograph of highly polished surface of ECO7175v1-T74 under dark field imaging to highlight particles on the surface.

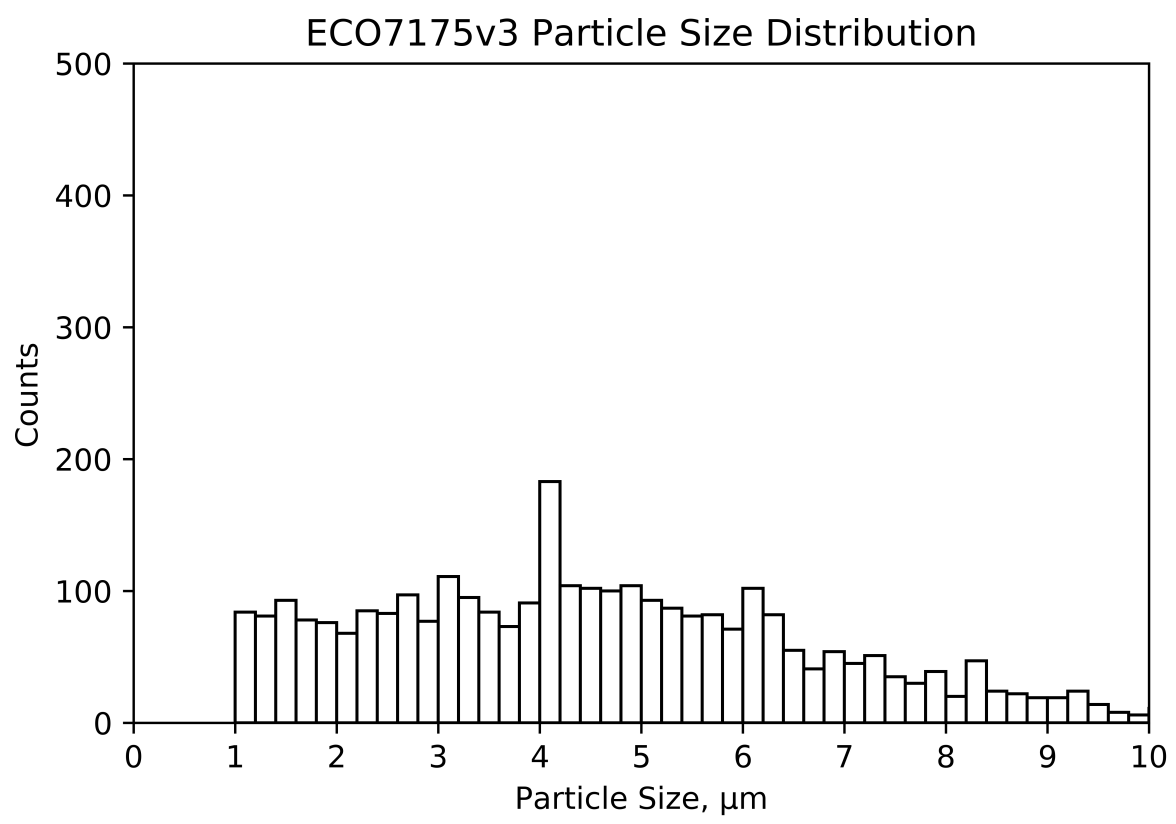

Figure 5.19: Particle size distribution of ECO7175v3-T74. 


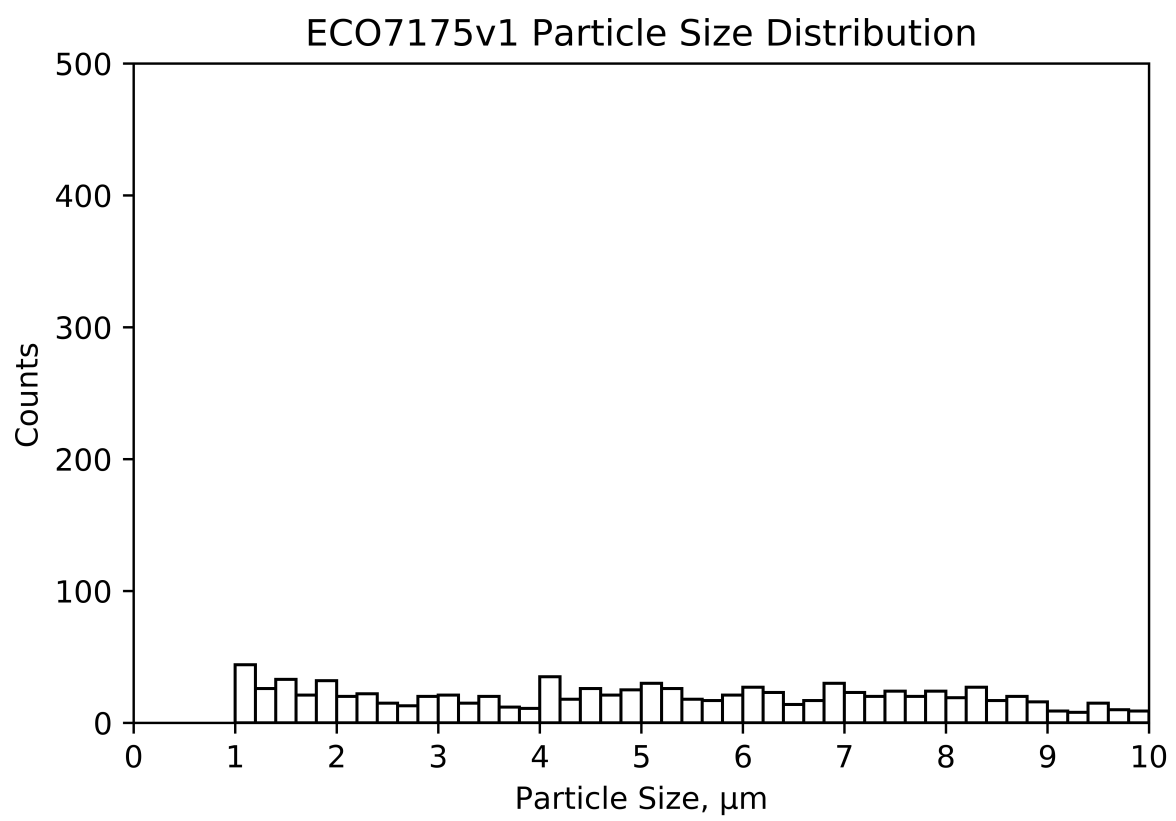

Figure 5.20: Particle size distribution of ECO7175v1-T74.

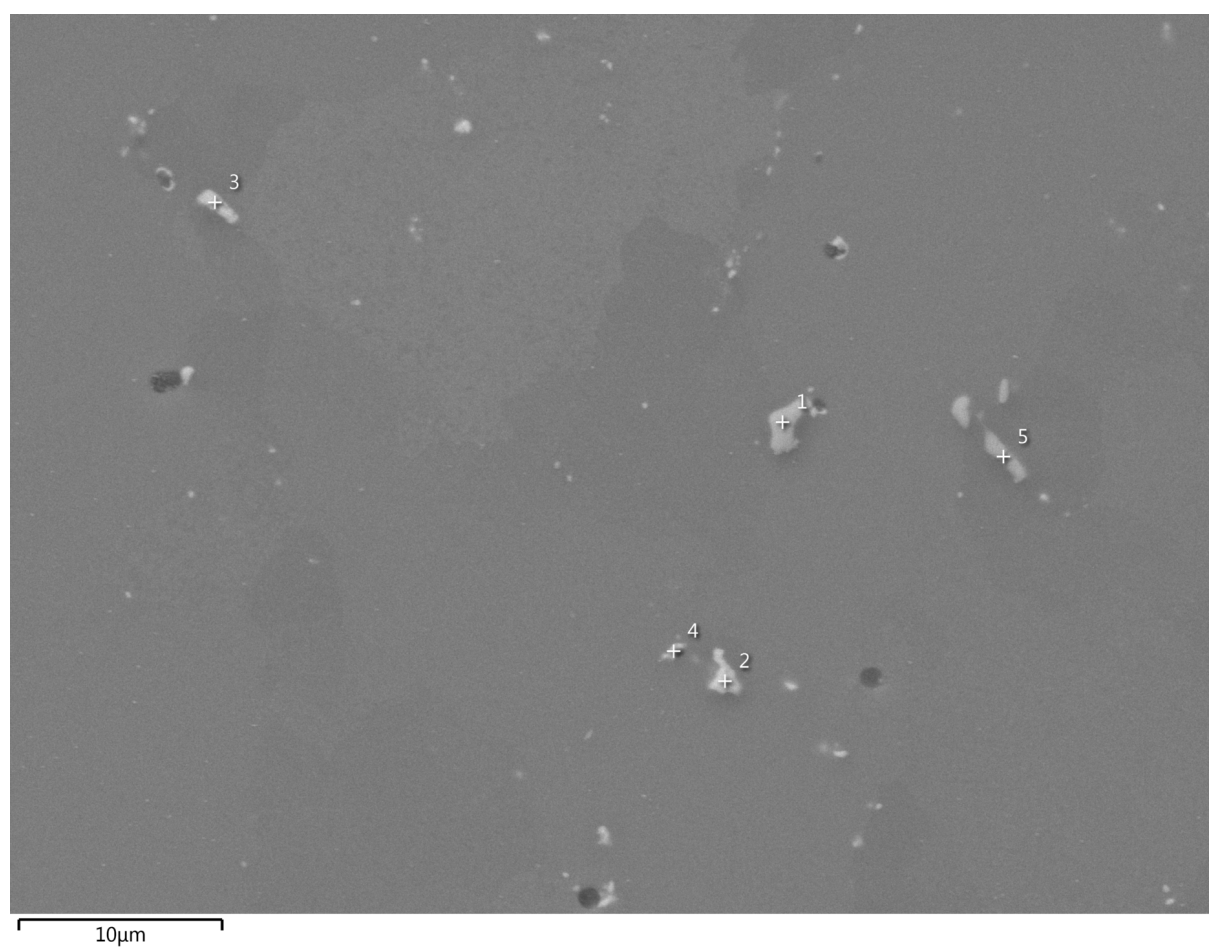

Figure 5.21: Precipitates points of interests chosen for EDX (Energy-dispersive $\mathrm{X}$-ray spectroscopy) analysis. 


\section{Chapter 6}

\section{ECO7175v2-T74}

Table 6.1 shows the allocation of specimens used for fatigue testing of ECO7175v2 to maintain the desired $\approx 5 \%$ coefficient of variation with a confidence of $90 \%$ for the fatigue testing. Its composition is given in Table 6.2 and its tensile properties was given previously in Table 4.3 .

Table 6.1: Allocation of Specimens for ECO7175v2-T74.

\begin{tabular}{cc}
\hline Stress Amplitude (MPa) & Number of Specimens \\
\hline 482 (85\% UTS) & 10 \\
$425(75 \%$ UTS $)$ & 10 \\
$369(65 \%$ UTS $)$ & 10 \\
$312(55 \%$ UTS $)$ & 14 \\
$255(45 \%$ UTS $)$ & 9 \\
$198(35 \%$ UTS $)$ & 2 \\
$142(25 \%$ UTS $)$ & 2 \\
\hline
\end{tabular}

The fatigue test results are tabulated in Table 6.3. Fatigue test statistics for stress amplitudes of $425 \mathrm{MPa}, 312 \mathrm{MPa}$, and $255 \mathrm{MPa}$ have a coefficient of variation of more than the desired 5\%. Fig. 6.1 showed the S-N curve for ECO7175v2. The majority of cycles to failure above $200 \mathrm{MPa}$ were between $10^{3}$ and $10^{7}$ cycles. The fatigue strength of the material at $5 \times 10^{7}$ cycles was difficult to determine for this variant and will require additional specimens to be subjected to fatigue testing around $40 \%$ of its tensile strength. Fig. 6.2 compares the S-N curve of ECO7175v2 with ECO7175v1 
Table 6.2: Manufactured Composition of Aluminum ECO7175v2-T74.

\begin{tabular}{cc}
\hline Element & Composition \% \\
\hline Aluminum (Al) & Balance \\
Calcium (Ca) & 0.0252 \\
Chromium (Cr) & 0.22 \\
Copper $(\mathrm{Cu})$ & 1.37 \\
Iron (Fe) & 0.08 \\
Magnesium (Mg) & 2.34 \\
Manganese (Mn) & 0.01 \\
Silicon (Si) & 0.05 \\
Titanium (Ti) & 0.036 \\
Zinc $(\mathrm{Zn})$ & 5.63 \\
\hline
\end{tabular}

and ECO7175v3.

Table 6.3: Statistical Analysis of Fatigue Testing for ECO7175v2-T74.

\begin{tabular}{c|ccc}
\hline Stress Amplitude (MPa) & Avg. Log Cycles & St.D. & Coeff. of Variation \% \\
\hline 482 (85\% UTS) & 3.724 & 0.084 & 2.3 \\
425 (75\% UTS) & 4.111 & 0.135 & 3.3 \\
369 (65\% UTS) & 4.460 & 0.109 & 2.4 \\
312 (55\% UTS) & 5.031 & 0.376 & 7.5 \\
255 (45\% UTS) & 5.316 & 0.313 & 5.9 \\
198 (35\% UTS) & Run-out (Over $5 \times 10^{7}$ Cycles) \\
$142(25 \%$ UTS) & Run-out (Over $5 \times 10^{7}$ Cycles) \\
\hline
\end{tabular}




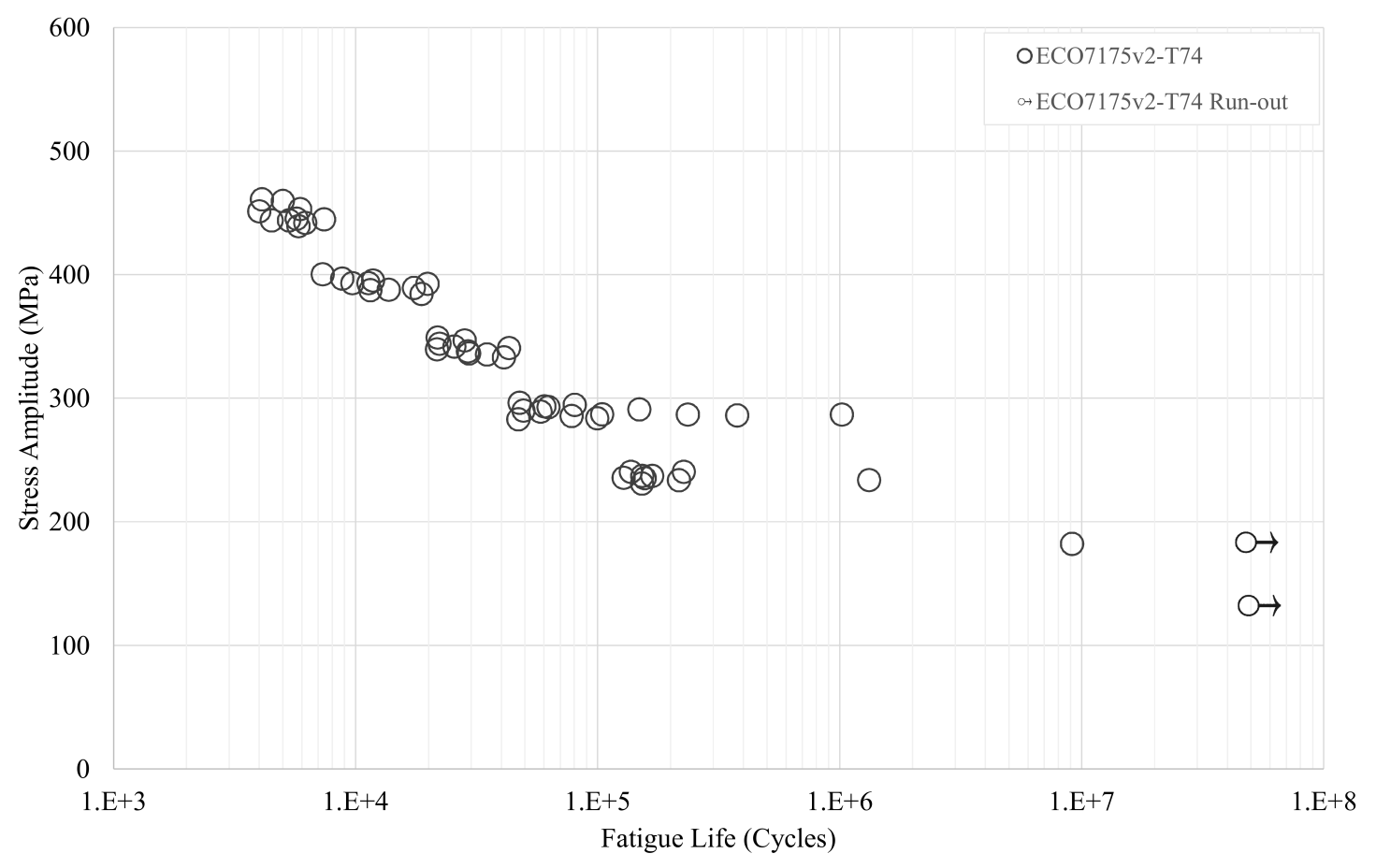

Figure 6.1: S-N data plot of aluminum ECO7175v2-T74. 


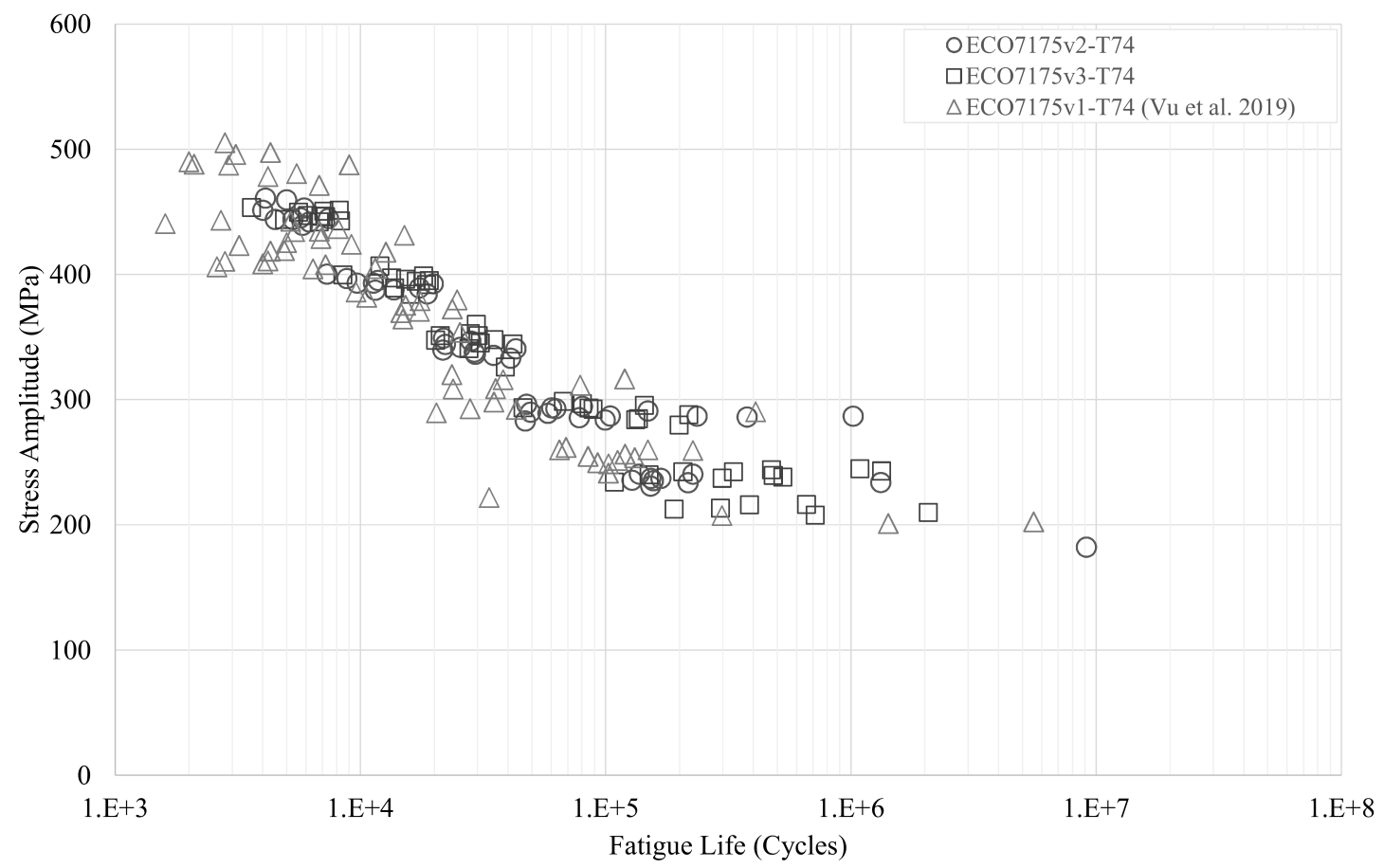

Figure 6.2: S-N data plot of aluminum ECO7175v1-T74 [38], ECO7175v2-T74, and ECO7175v3-T74. 


\subsection{Fracture Analysis}

Fracture surfaces between specimens at various stress levels were compared. Fractography results show that ECO7175v2 has similar fracture characteristics to those of ECO7175v1 that are subjected to $66 \%$ and below of its tensile strength. ECO7175v2 typically have one or two primary crack initiation points where long crack propagation bands propagate from. Like ECO7175v1, these propagation bands are capable of reaching halfway or more through the specimens. Comparing it to ECO7175v3, once again it can be seen that ECO7175v3 have unique fracture characteristics compared to ECO7175v1 and ECO7175v2 (Fig. 6.4). Under the SEM, striations were found and measured. The striations have a width of approximately $1.070 \mu \mathrm{m}$, on average. The striation width is comparable to ECO7175v3 but considerably higher than ECO7175v1. A SEM image of the striations is shown in Fig. 6.5. No further SEM analysis and EDX analysis were done for ECO7175v2. 


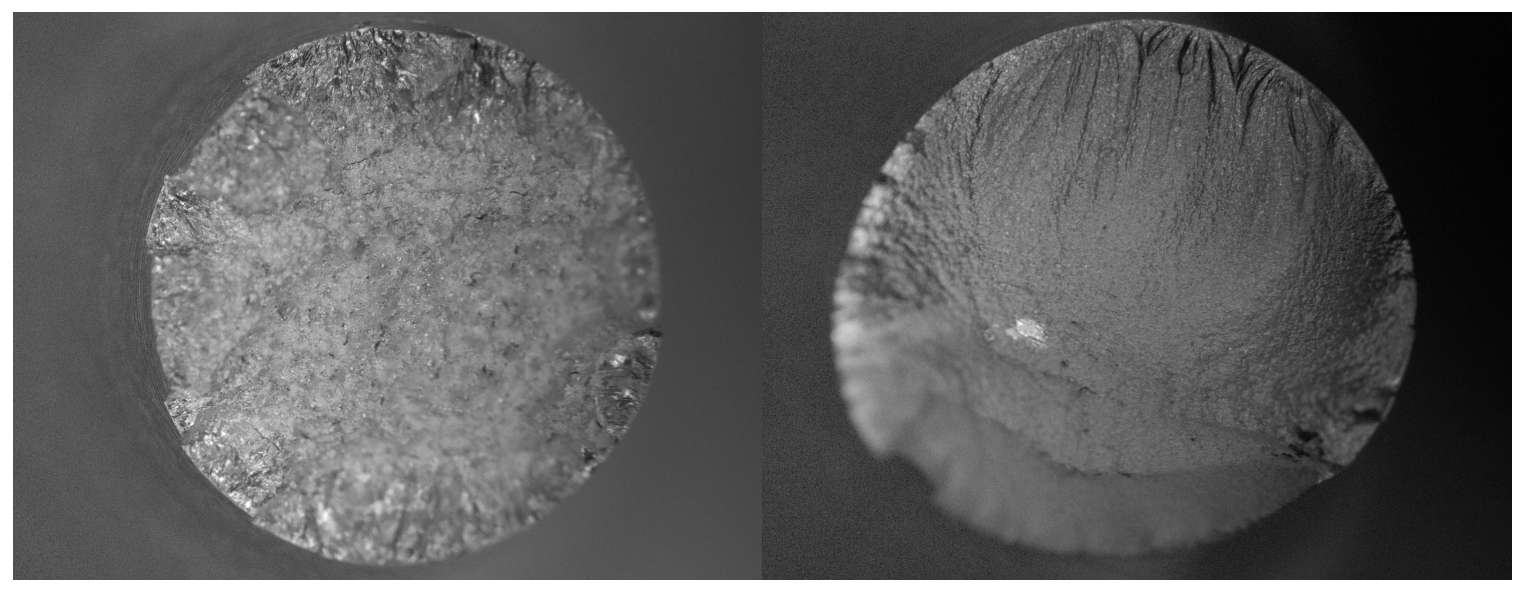

Figure 6.3: Specimens subjected to 75\% UTS (right) have fracture characteristics that are well defined with long propagation bands, clear crack initiation sites, and have failure modes that are predominately ductile near the surface while brittle towards the center. Specimen subjected to $85 \%$ UTS (left) has similar characteristics to specimens subjected to $75 \%$ and higher on ECO7175v1-T74 with many crack initiation points around the rim and short propagation bands. 


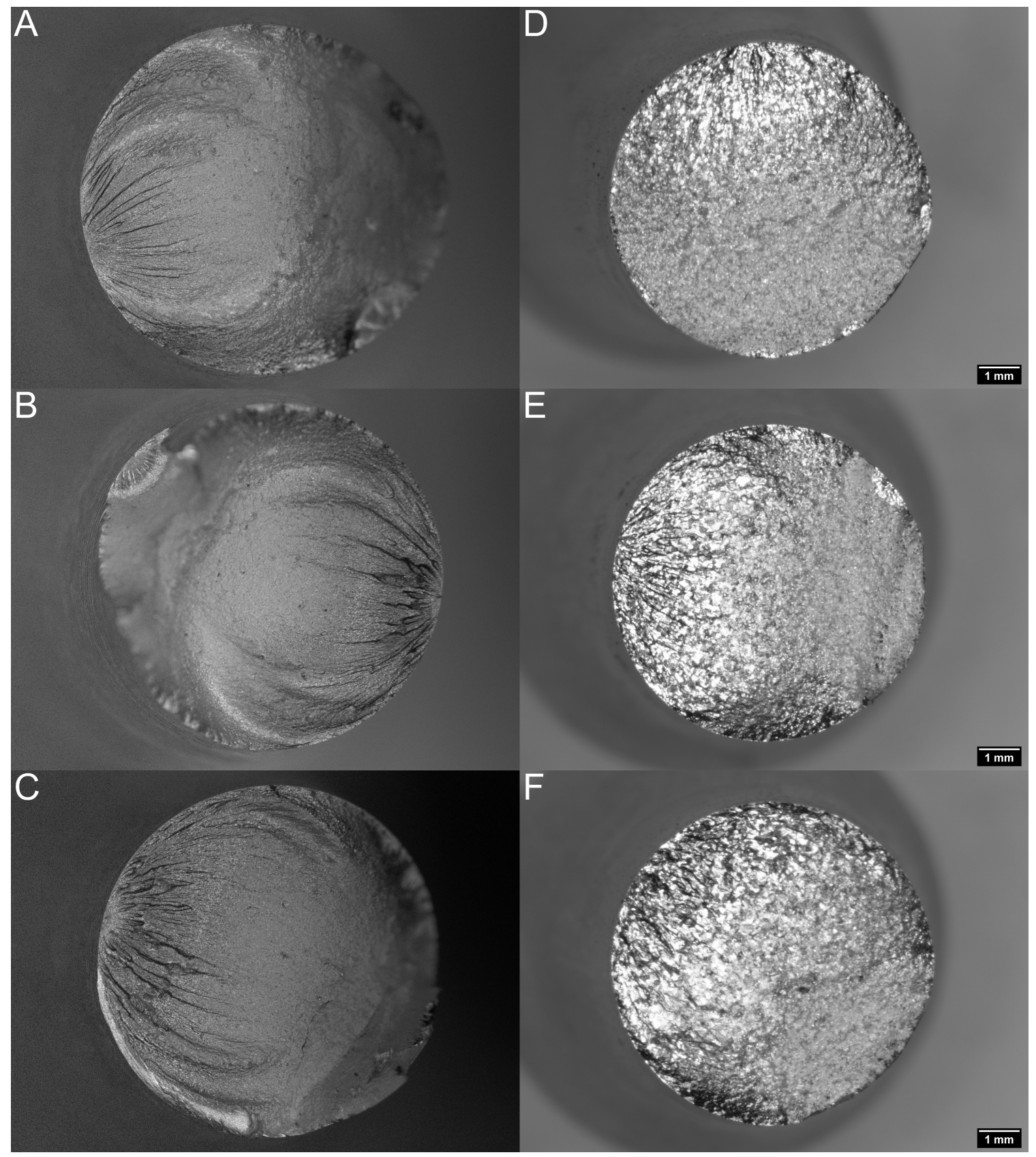

Figure 6.4: Left three are ECO7175v2-T74 specimens subjected to 65\% UTS (A), $55 \%$ UTS (B), and 45\% UTS (C). Right three are ECO7175v3-T74 specimens subjected to similar stresses at $67 \%$ UTS (D), $57 \%$ UTS (E), and $47 \%$ UTS (F). 


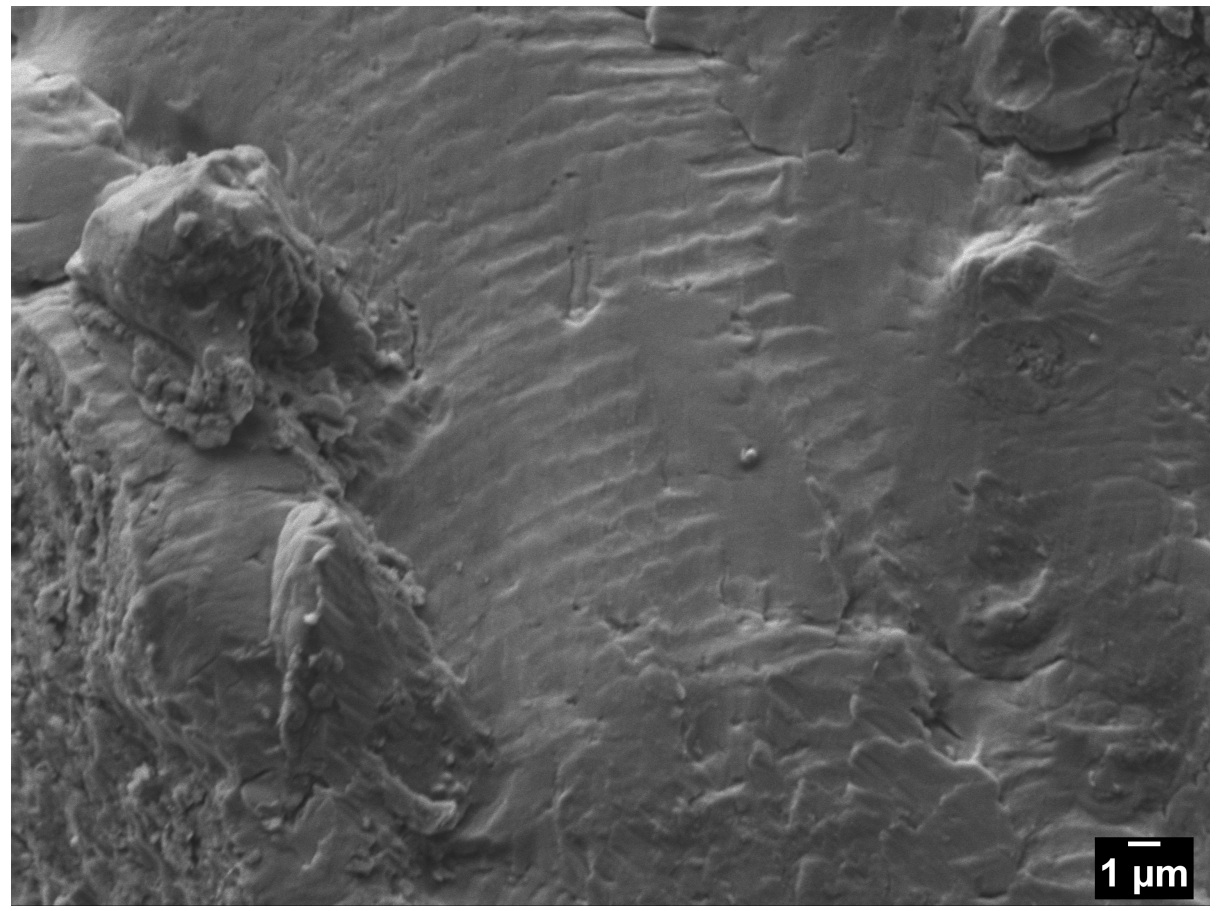

Figure 6.5: SEM photograph of striations for ECO7175v2-T74 subjected to 75\% UTS. The striations, on average, have a width of approximately $1.070 \mu \mathrm{m}$. The striation width is comparable to those of ECO7175v3-T74 but still considerably higher than those of ECO7175v1-T74. 


\subsection{Metallography}

The polished surfaces of ECO7175v2 were analyzed for particles. At 100x, unlike ECO7175v1 (Fig. 5.18) and ECO7175v3 (Fig. 5.17), the particles were very hard to see. The particles were hard to see even in dark field imaging (Fig. 6.6). They were hard to see because the particles are much smaller compared to those seen in the other two variants. At 200x magnification, they are now easily visible (Fig. 6.7). The particles were calculated to have a volume fraction of $0.48 \%$, considerably lower than the other two variants. However, under Nomarski imaging, Fig. 6.8, the particles are revealed to be primarily pores. Very little precipitates are actually seen. The particle distribution for ECO7175v2-T74 is shown in Fig. 6.9. 


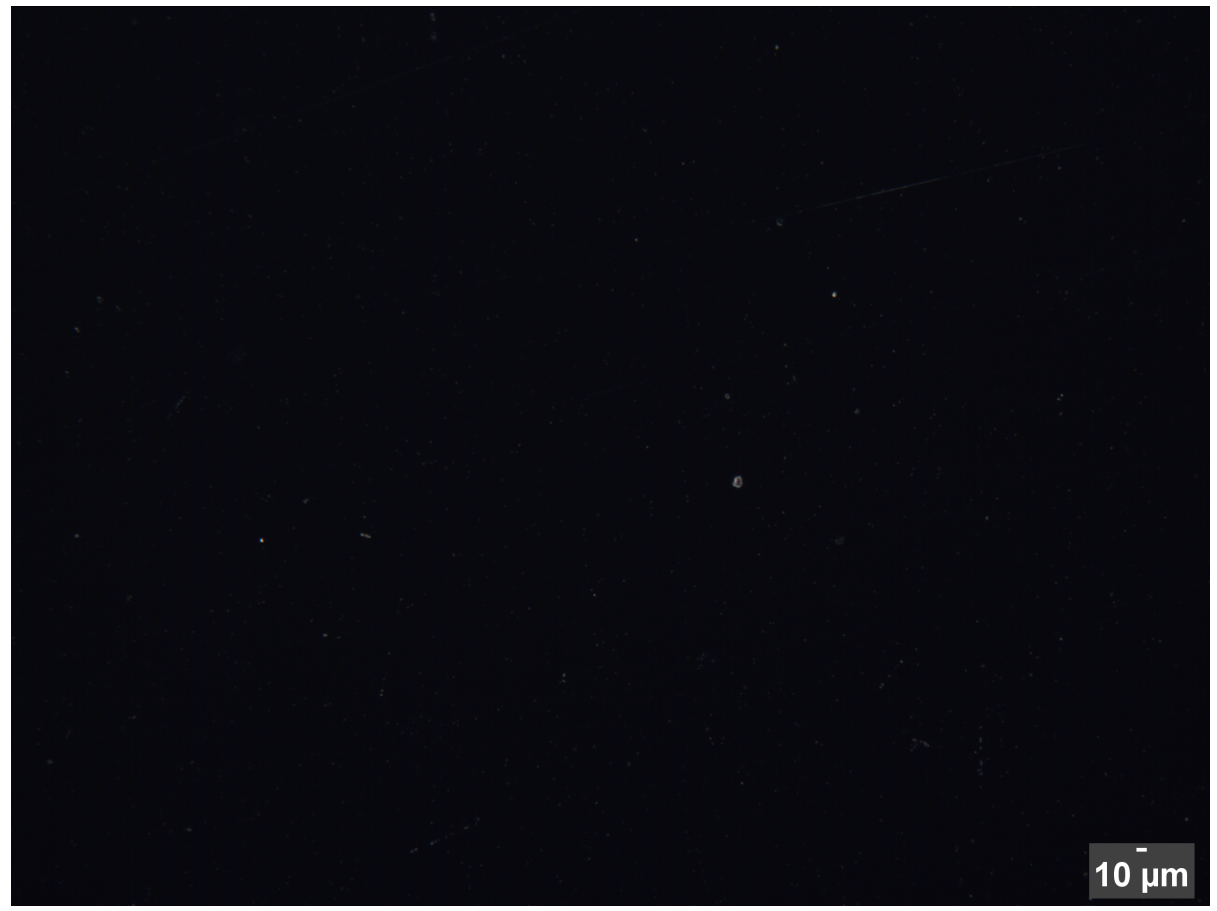

Figure 6.6: Metallograph of highly polished surface of ECO7175v2-T74 under dark field imaging to highlight the particles on the surface. At 100x magnification. Compared to the other two variants, the particles in ECO7175v2-T74 were incredibly hard to see as they are considerably smaller. 


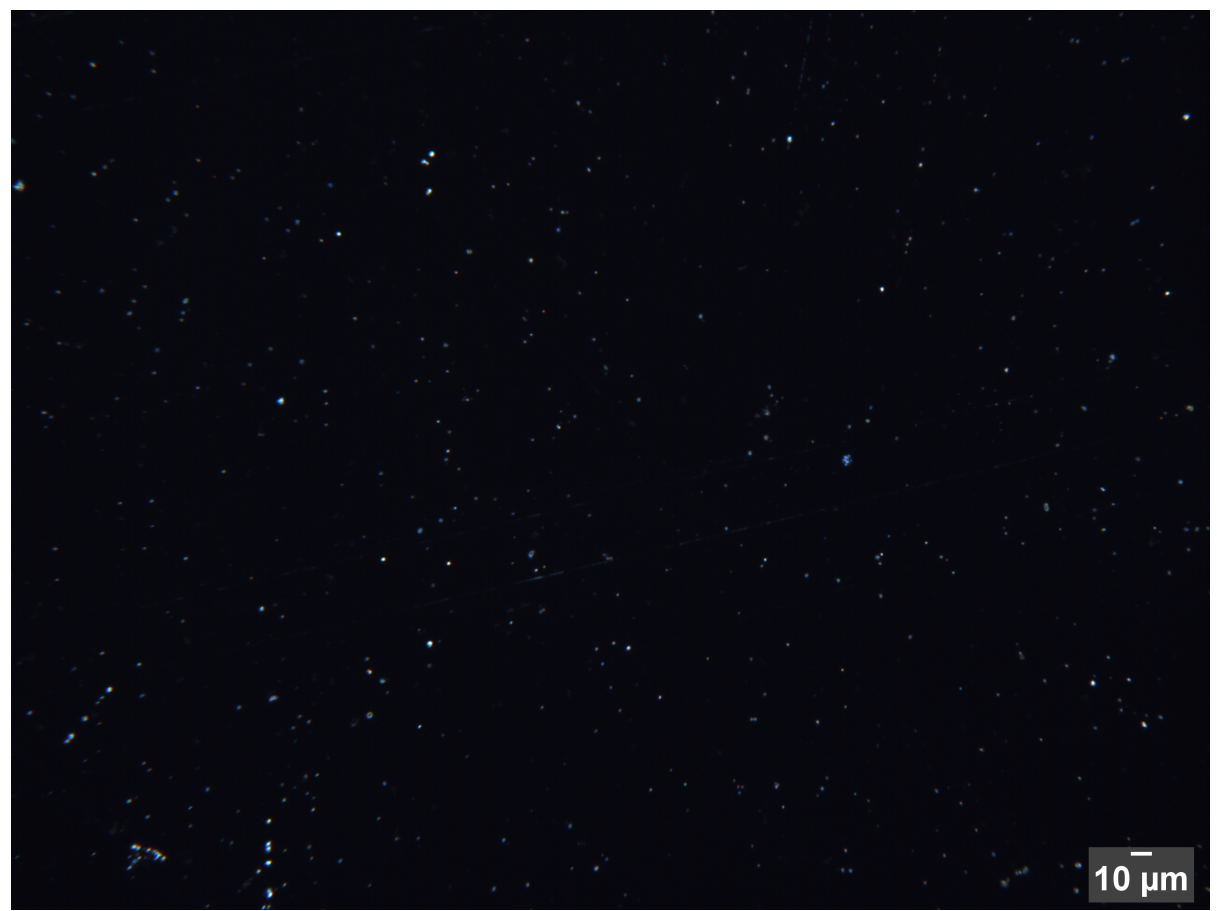

Figure 6.7: Metallograph of highly polished surface of ECO7175v2-T74 under dark field imaging to highlight the particles on the surface. At 200x magnification. 


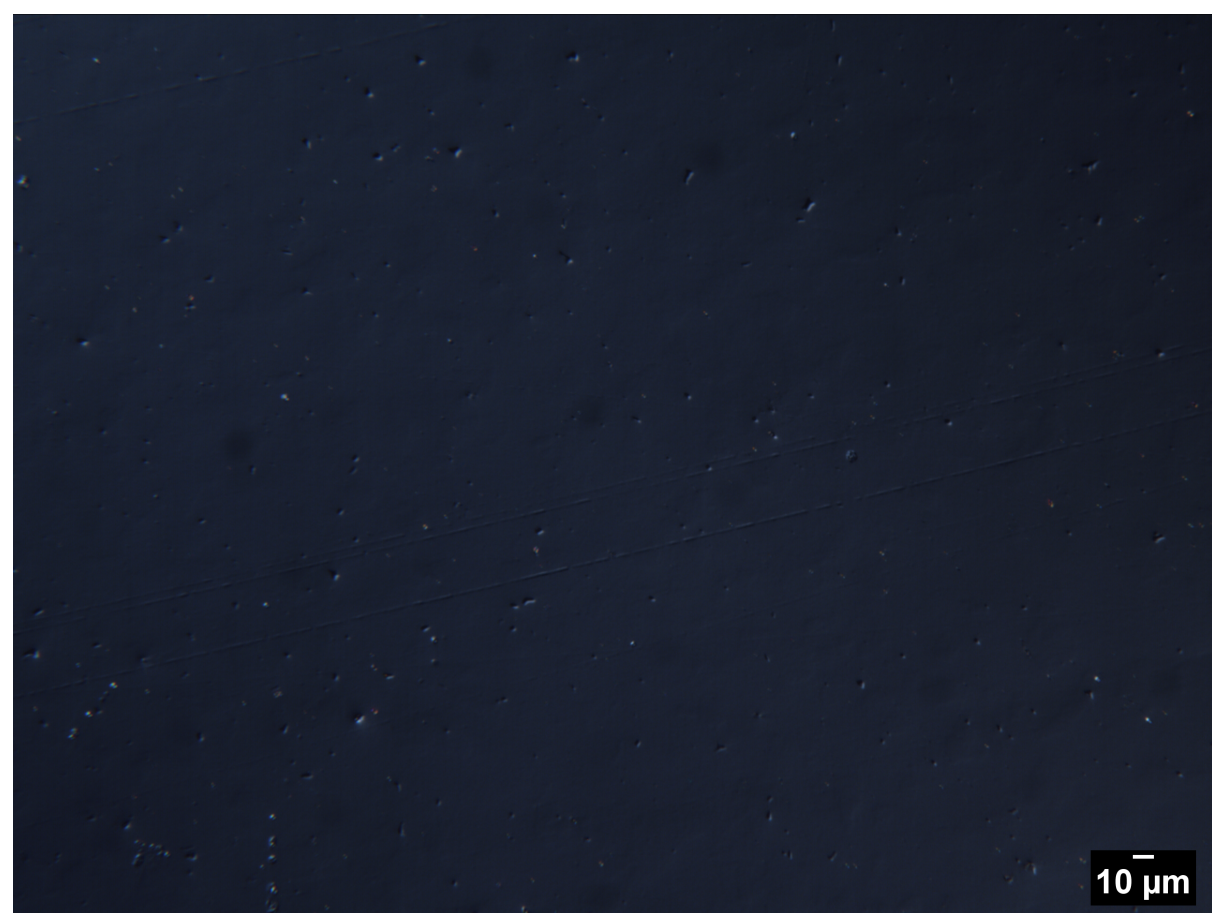

Figure 6.8: ECO7175v2-T74 specimens under Nomarski showed that compared to the other two variants, there were very little precipitates. The majority of what was noticed under the dark field imaging were in fact, pores. At 200x magnification.

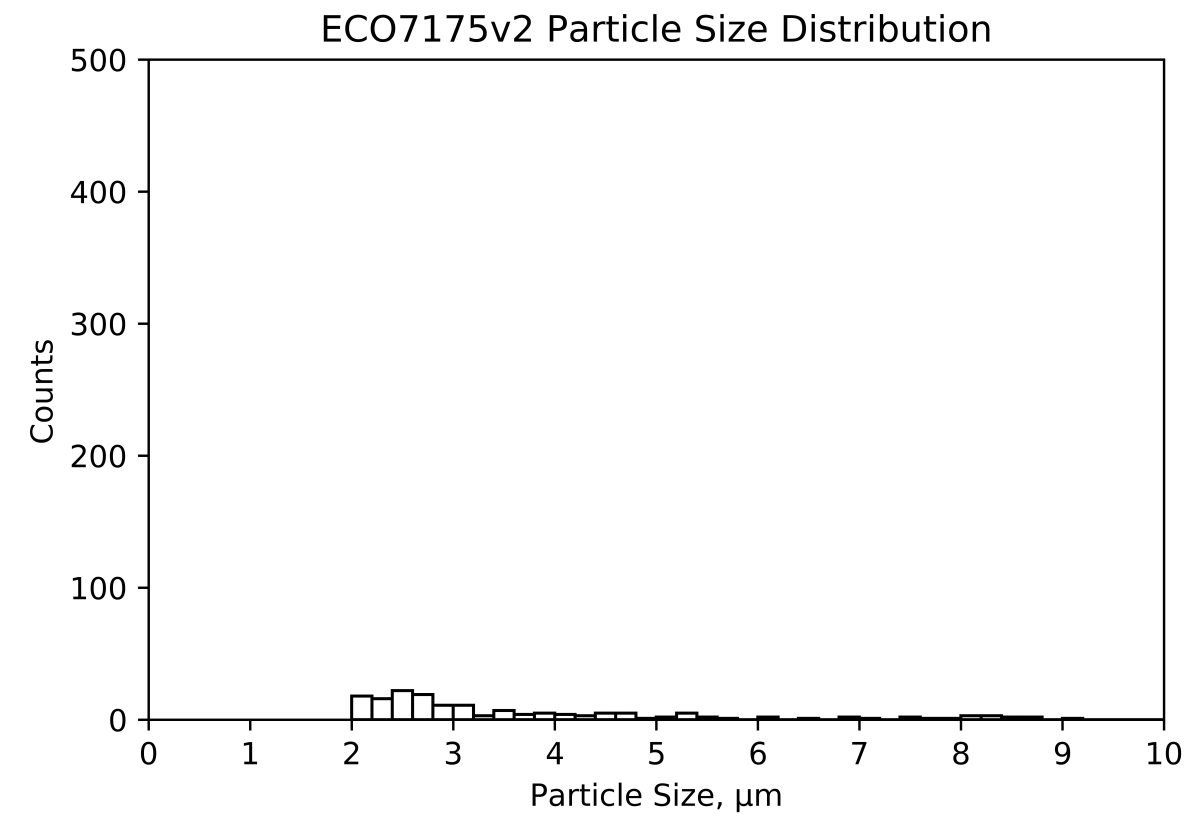

Figure 6.9: Particle size distribution of ECO7175v2-T74. 


\subsection{Basquin's Equation}

The fatigue life data were then fitted to Basquin's equation to determine its constants (Fig. 6.10). The fatigue strength coefficient, $\sigma_{F}^{\prime}$, was determined to be 1547.1 MPa and the fatigue strength exponent, $b$, was determined to be -0.145 . Among the three variants, it has the lowest fatigue strength coefficient.

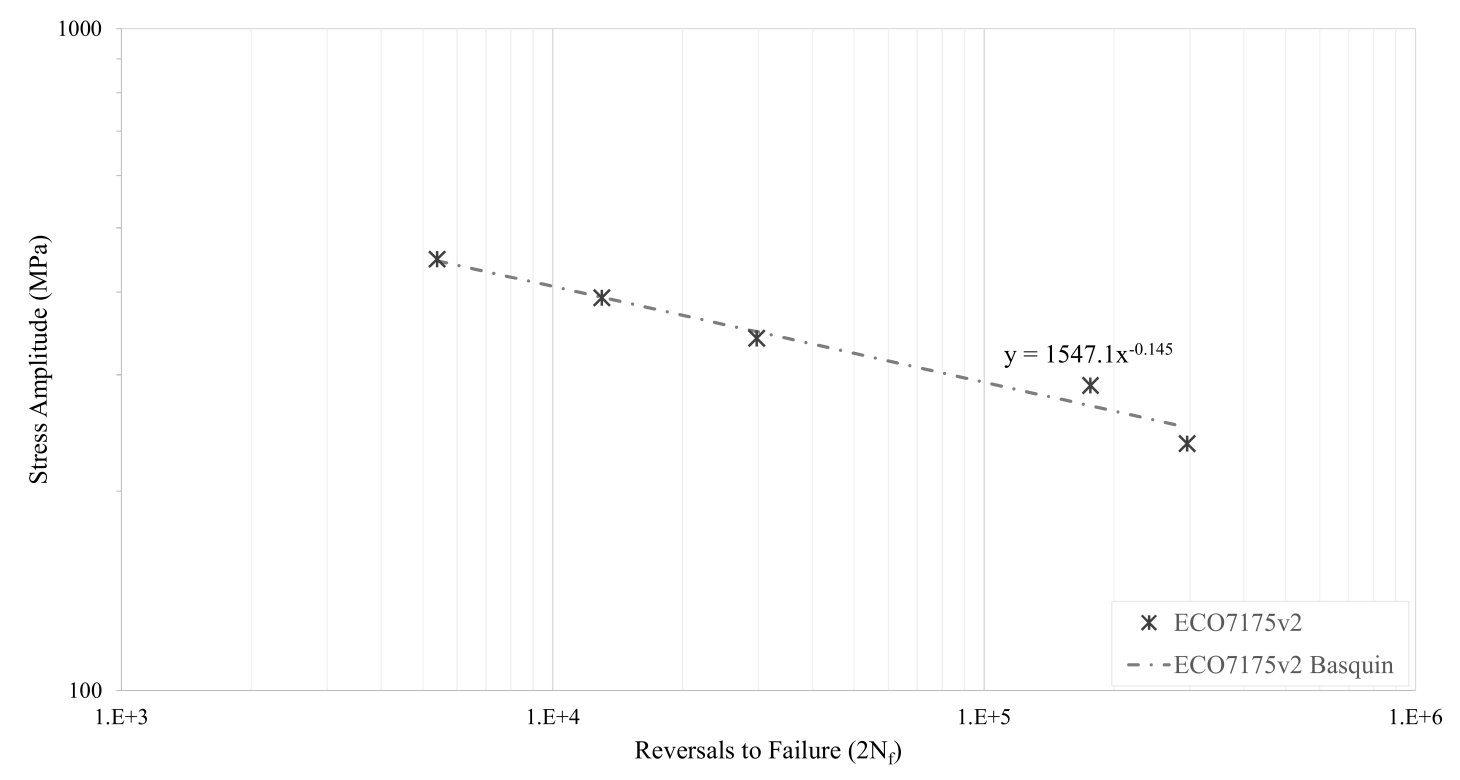

Figure 6.10: Fatigue data for ECO7175v2-T74 curve-fitted to Basquin's equation to determine the material's fatigue strength coefficient, $\sigma_{F}^{\prime}$, and fatigue strength exponent, $b$. 


\section{Chapter 7}

\section{Discussions}

\subsection{General Analysis}

The compositions of all three ECO7175 variants are shown once more in Table 7.1. Bold elements are elements that were added intentionally while non-bold elements existed as impurities within commercial aluminum ingots. Copper, magnesium, and zinc were added to the ECO7175 aluminum following the standard specification for 7175 . The content of zinc is slightly higher than the specification for ECO7175v1 due to unintentional tolerances during the manufacturing of the material for this research. The primary differences between each ECO7175 variant are the calcium, chromium, and titanium content. Titanium was added as a grain refiner [44], calcium was added for melt cleanliness, and chromium was added to ECO7175v1 and ECO7175v2 within the specification for aluminum 7175. However, chromium was not added to ECO7175v3. Not in excess of $0.35 \mathrm{wt} \%$, chromium is used to prevent grain growths in aluminummagnesium alloys and to prevent recrystallization in aluminum-magnesium-zinc and aluminum-magnesium-silicon alloys [43]. The effect of added chromium is seen in Fig. 7.1. ECO7175v1 and ECO7175v2 are shown to have elongated grains in the TD direction while ECO7175v3 is shown to have considerably larger and equiaxed grains (uniform grain size in all directions). 
Table 7.1: Manufactured composition of aluminum ECO7175 and standard reference aluminum 7175. Bold elements were intentionally added. Non-bold elements existed as natural impurities in commercial pure aluminum ingots. Compositions in wt\%.

\begin{tabular}{c|cccc}
\hline Element & ECO7175v1 & ECO7175v2 & ECO7175v3 & Ref.7175 \\
\hline $\mathrm{Al}$ & Balance & Balance & Balance & Balance \\
$\mathrm{Ca}$ & $\mathbf{0 . 0 4 1}$ & $\mathbf{0 . 0 2 5 2}$ & $\mathbf{0 . 0 3 1}$ & - \\
$\mathrm{Cr}$ & $\mathbf{0 . 2 1}$ & $\mathbf{0 . 2 2}$ & 0.002 & $\mathbf{0 . 1 8 - 0 . 2 8}$ \\
$\mathrm{Cu}$ & $\mathbf{1 . 7 4}$ & $\mathbf{1 . 3 7}$ & $\mathbf{1 . 2 8}$ & $\mathbf{1 . 2 - 2 . 0}$ \\
$\mathrm{Fe}$ & 0.08 & 0.08 & 0.07 & 0.20 \\
$\mathrm{Mg}$ & $\mathbf{2 . 7 3}$ & $\mathbf{2 . 3 4}$ & $\mathbf{2 . 4 2}$ & $\mathbf{2 . 1 - 2 . 9}$ \\
$\mathrm{Mn}$ & 0.01 & 0.01 & 0.01 & 0.10 \\
$\mathrm{Si}$ & 0.04 & 0.05 & 0.05 & 0.15 \\
$\mathrm{Ti}$ & $\mathbf{0 . 0 2 8}$ & $\mathbf{0 . 0 3 6}$ & $\mathbf{0 . 0 4 5}$ & 0.10 \\
$\mathrm{Zn}$ & $\mathbf{5 . 9 5}$ & $\mathbf{5 . 6 3 1}$ & $\mathbf{5 . 5 5}$ & $\mathbf{5 . 1 - 5 . 6}$ \\
\hline
\end{tabular}

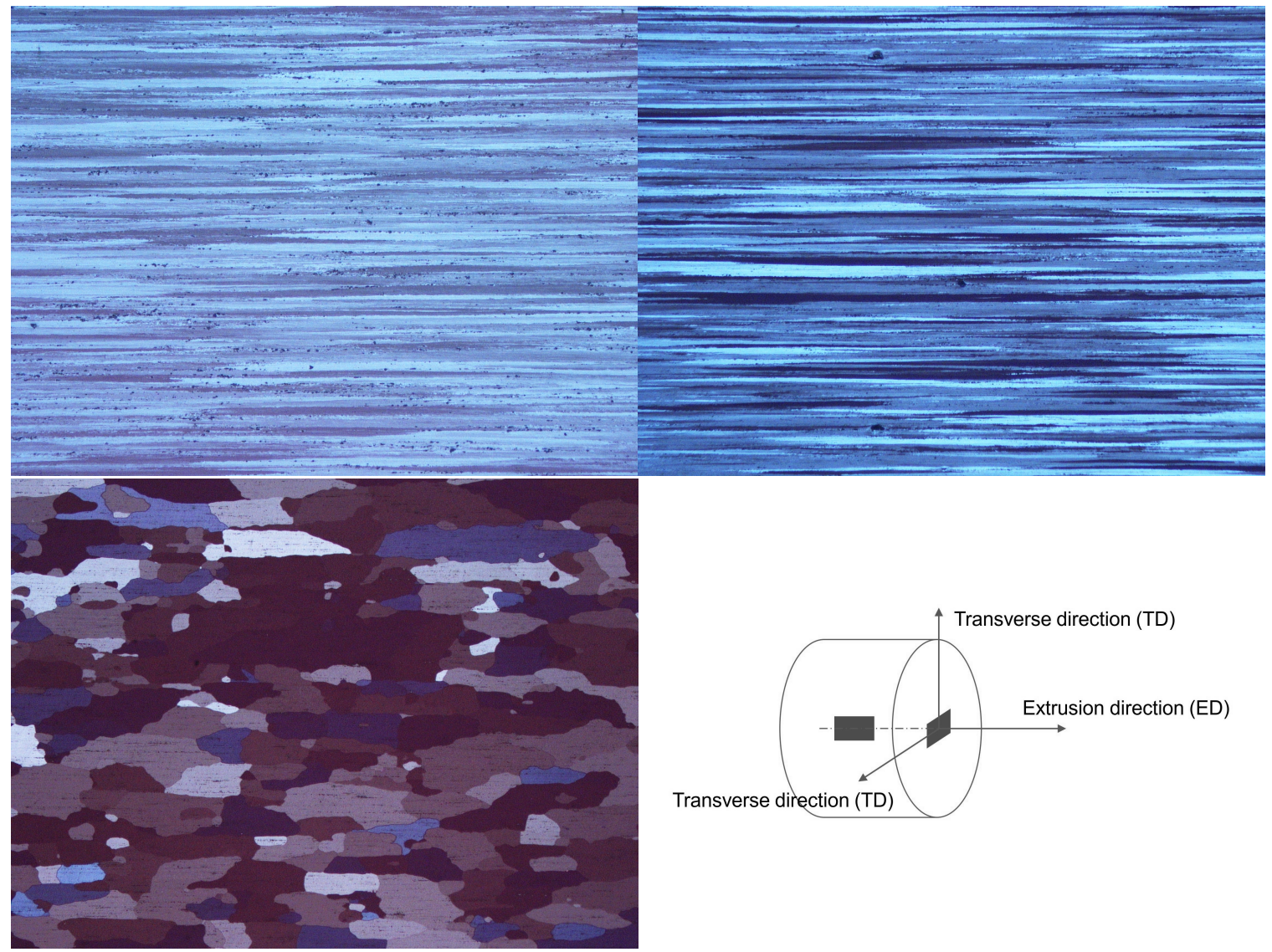

Figure 7.1: Grains in the direction of TD for ECO7175v1 (top left) and ECO7175v2 (top right) are highly elongated while grains for ECO7175v3 (bottom left) are larger and equiaxed in all directions. 


\subsection{Fatigue and Fracture Analysis}

All variants of ECO7175 extruded aluminum alloy with T74 temper were subjected to fully reversed bending load and investigated to find their fatigue characteristics. The S-N curve shows that the fatigue life of ECO7175v1 can exceed $10^{7}$ cycles with a fatigue strength of approximately $207 \mathrm{MPa}$ or less, about $36 \%$ of its tensile strength. For ECO7175v3, the S-N curve shows that its fatigue life can exceed $5 \times 10^{7}$ cycles with a fatigue strength of approximately $220 \mathrm{MPa}$ or less, about $40 \%$ of its tensile strength. This represents an improvement of approximately $6 \%$ over ECO7175v1. The S-N curve was also graphed for ECO7175v2, but it is difficult to determine its fatigue strength and may require additional specimens to be fatigue tested at approximately $40 \%$ of its tensile strength. However, it is still shown that the fatigue strength of ECO7175v2 approaches approximately $200 \mathrm{MPa}$, just like the other two variants.

Fracture analysis of ECO7175 indicates that crack surfaces of specimens at high stress levels typically exhibits mixed failure modes in which the outer rims are predominately ductile and the inner surfaces are predominately brittle. In addition, fracture surfaces for ECO7175v1 and ECO7175v3 at higher stress levels typically have more crack initiation points. Conversely, both ECO7175v1 and ECO7175v3 at lower stress levels have fewer crack initiation points. ECO7175v3 specimens that were subjected to stress levels of $67 \%$ of its tensile strength and lower have fracture surfaces that 
generally have softly defined fracture features and propagation bands that are hard to discern by the unaided eye. ECO7175v1 and ECO7175v2, on the other hand, have fracture surfaces with sharply defined features and propagation bands that are easily seen. Irrespective of the stress levels, ECO7175v1 and ECO7175v3 are shown to all have crack initiations points at the surface and no inclusions to act as stress concentrators were seen. In addition, striations for all variants were found and measured (Table 7.2). Each striation represents how much the crack propagates, or "ripped," per stress cycle.

Table 7.2: ECO7175's Striations' Width.

\begin{tabular}{cc}
\hline Variant & Average Striation Width $(\mu \mathrm{m})$ \\
\hline ECO7175v1-T74 & 0.481 \\
ECO7175v2-T74 & 1.070 \\
ECO7175v3-T74 & 1.164 \\
\hline
\end{tabular}

\subsection{Metallography}

Grain sizes for ECO7175 were observed and measured. The grain size of ECO7175v3 is approximately $78 \mu \mathrm{m}$ on average. The grain sizes for ECO7175v1 and ECO7175v2 are approximately $10 \mu \mathrm{m}$ on average. The larger grain size for ECO7175v3 is caused by the lack of chromium, which would have suppressed recrystallization during its heat treatment. Particles on the polished specimens of all variants were measured and counted. The particle size distribution indicates ECO7175v3 have 
significant higher counts of precipitates with a calculated volume fraction of approximately $2.5 \%$. On the contrary, the particle size distribution indicates ECO7175v1 has lower counts of precipitates but slightly higher counts of larger precipitates compared to ECO7175v3. ECO7175v1 has a calculated volume fraction of approximately $1.6 \%$. Among the three variants of ECO7175, ECO7175v2 has the lowest volume fraction at $0.48 \%$. However, under Nomarski imaging, it shows that the particles observed on ECO7175v2 are generally pores. Basic EDX analysis was conducted on the precipitates for ECO7175v3 and ECO7175v1. The EDX analysis shows the precipitates are generally high concentrations of $\mathrm{Fe}, \mathrm{Cu}, \mathrm{C}, \mathrm{Zn}, \mathrm{Mg}$, and $\mathrm{Ca}$. In addition, high concentrations of $\mathrm{Cr}$ are also found in ECO7175v1's precipitates.

\subsection{Reliability Analysis}

\section{Estimating Reliability}

The reliability and cumulative hazard function of each variant of ECO7175 were estimated using the Kaplan-Meier and Nelson-Aalen estimator. The results were then compared. The graph of ECO7175 at 85\% UTS is presented in Fig. 7.2, 75\% UTS (Fig.7.3 ), 65\% UTS (Fig. 7.4), 55\% UTS (Fig. 7.5), and 45\% UTS (Fig. 7.6). At any given fatigue life cycle and stress level, it is shown that ECO7175v1 has considerably lower reliability compared to ECO7175v2 and ECO7175v3. 


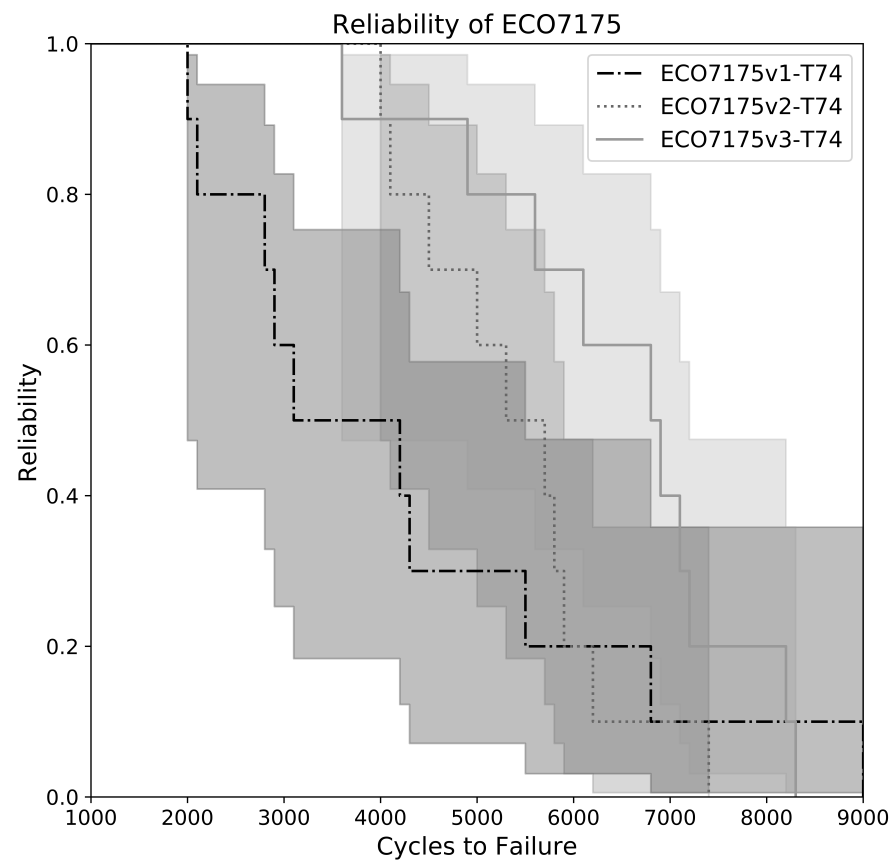

Figure 7.2: Kaplan-Meier estimator used to estimate the reliability of ECO7175T74 at $85 \%$ UTS. The shade represents the $95 \%$ confidence interval. Although the confidence intervals are overlapping, the estimates show that in general, at any given fatigue life cycle and stress level, ECO7175v1 has lower reliability when compared to the other variants. 


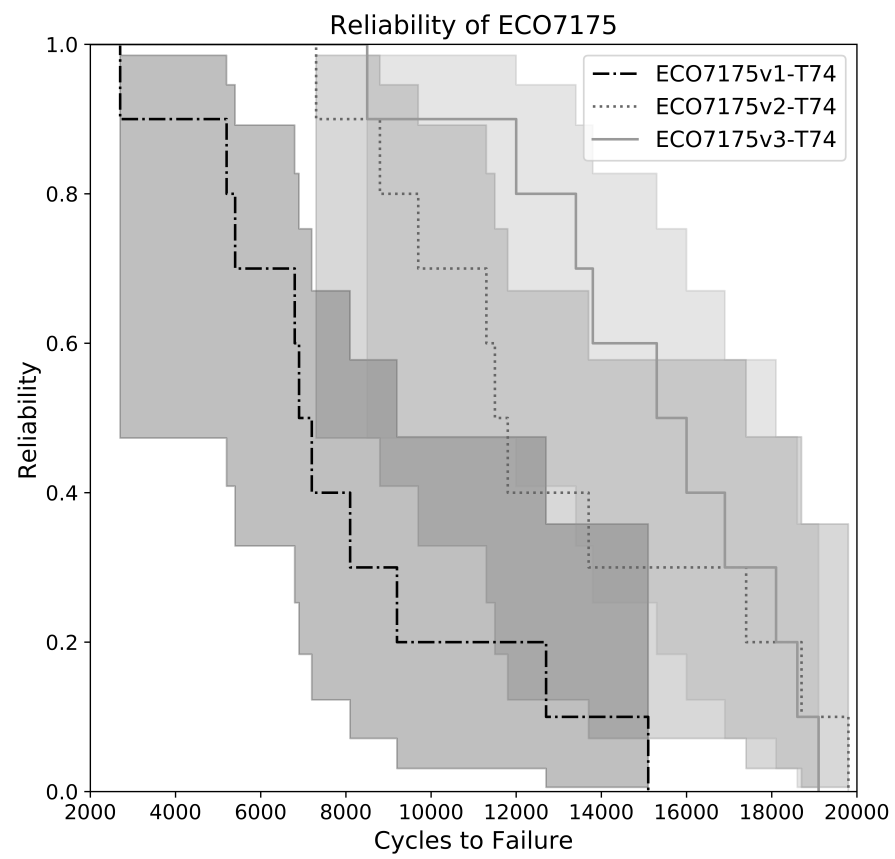

Figure 7.3: Kaplan-Meier estimator used to estimate the reliability of ECO7175-T74 at $75 \%$ UTS.

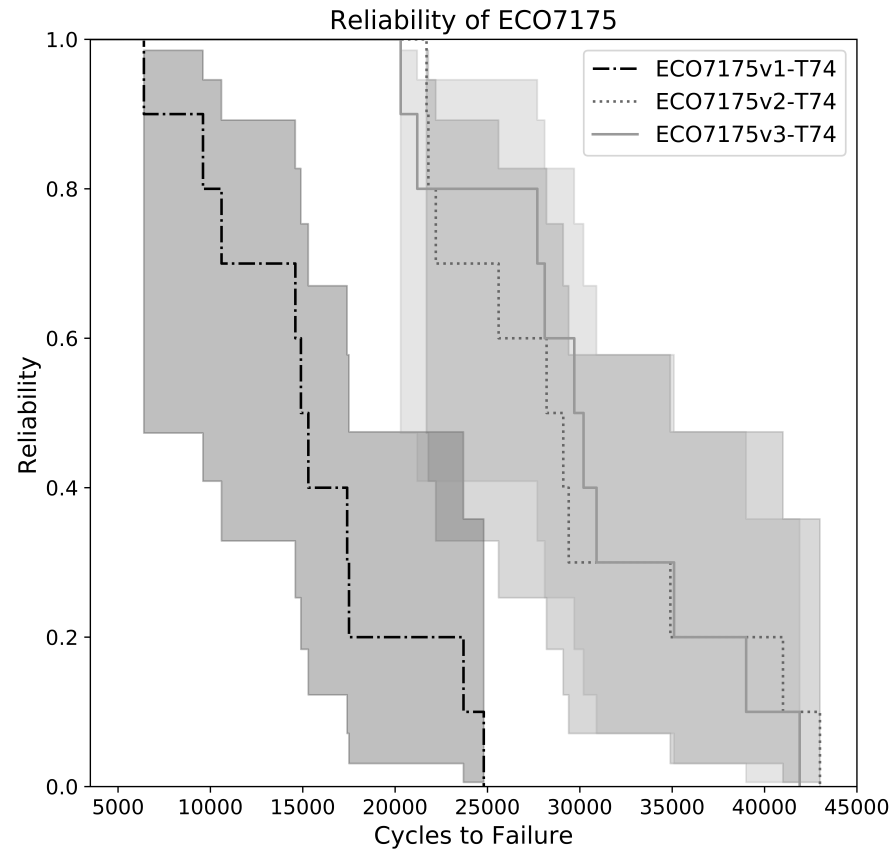

Figure 7.4: Kaplan-Meier estimator used to estimate the reliability of ECO7175-T74 at $65 \%$ UTS. 


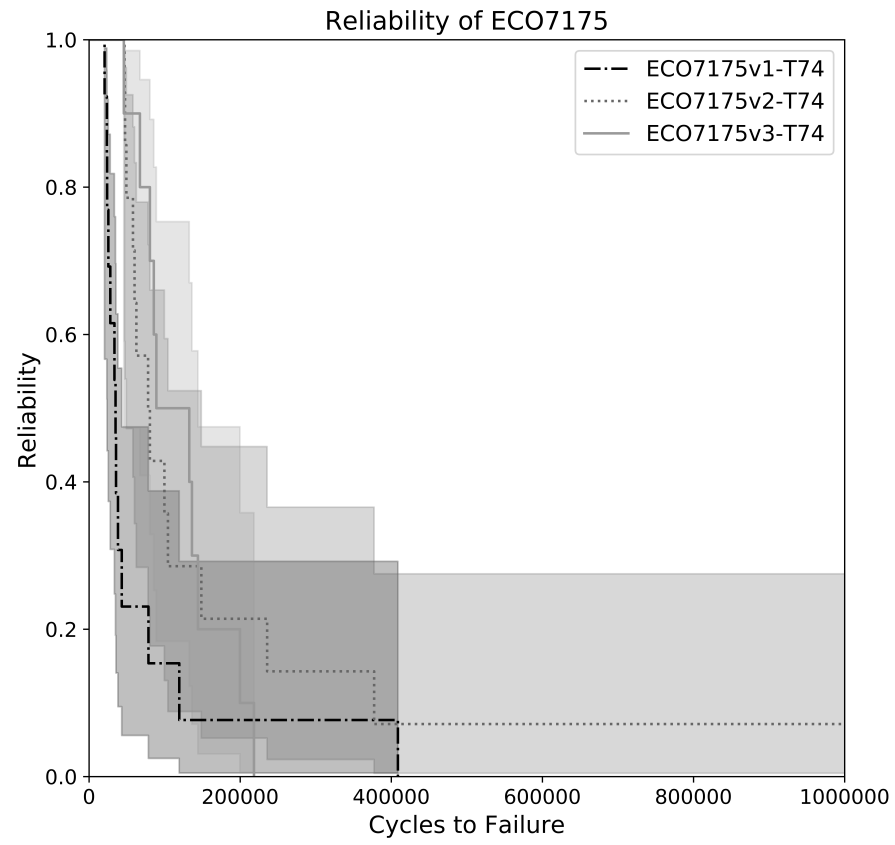

Figure 7.5: Kaplan-Meier estimator used to estimate the reliability of ECO7175-T74 at $55 \%$ UTS.

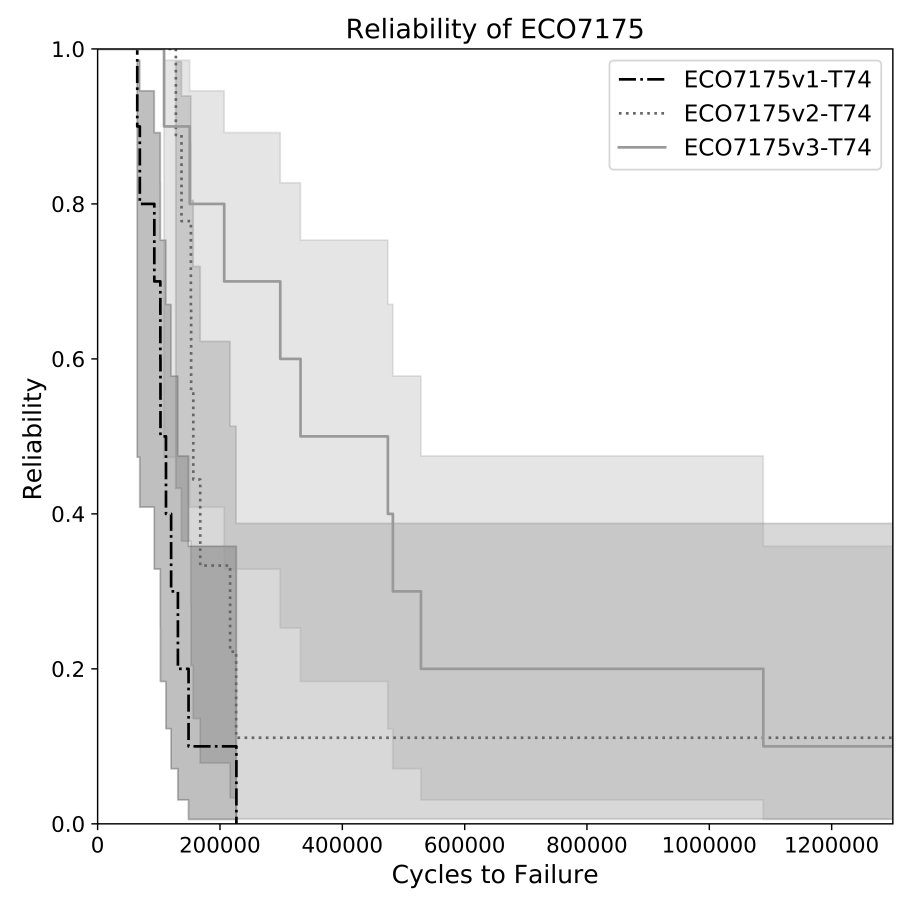

Figure 7.6: Kaplan-Meier estimator used to estimate the reliability of ECO7175-T74 at $45 \%$ UTS. 
The estimated reliability for all variants of ECO7175 was curve-fitted to the Weibull distribution. The results further emphasize that ECO7175v1 has lower reliability compared to the other variants at any given fatigue life cycle, such as in the case at $66 \%$ of their tensile strength (Fig. 7.7). When ECO7175v2 and ECO7175v3 were compared, once again at any given fatigue life cycle, ECO7175v3 is shown to have higher reliability at any given fatigue life cycle. A log-rank test may be used to give more conclusive comparisons. Table 7.3 show the full statistic parameters for the Weibull distribution at $65 \%$ of their tensile strength. $\alpha$ is set as 0.05 , representing $95 \%$ confidence (5\% error of rejecting the null hypothesis when it is actually true, Type I error). The hypothesis for the hypothesis testing is shown in Eq. 7.1. The data fit is good and as $\beta>1$, it suggests all failures are wear-out failures. 


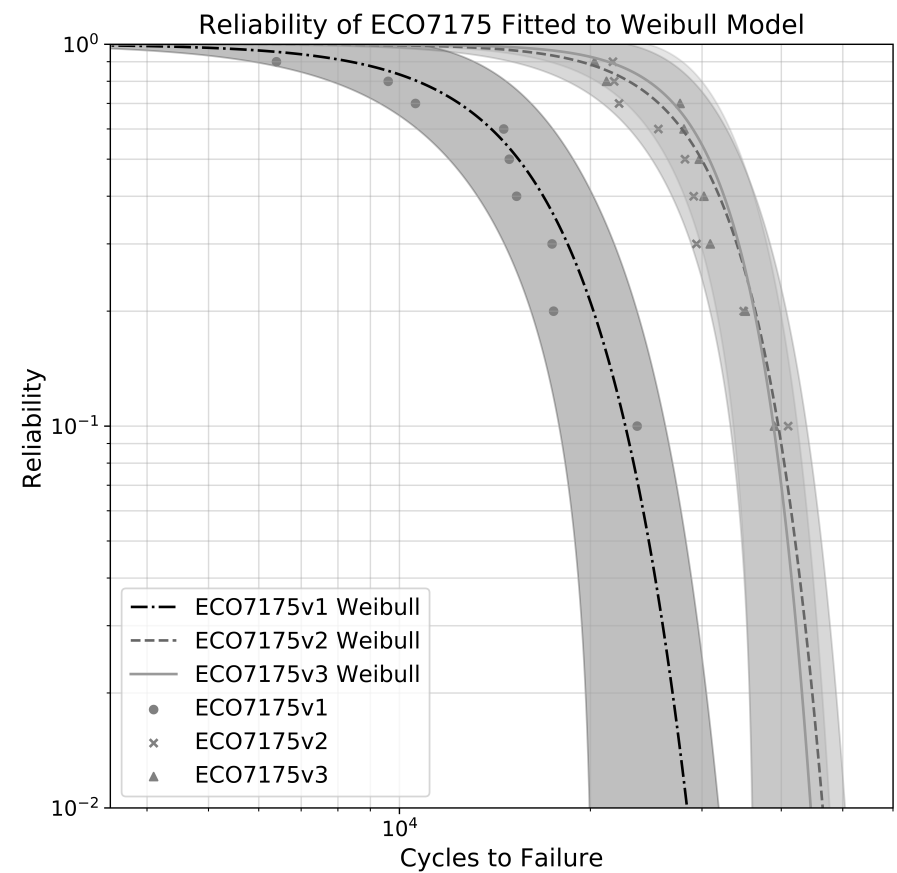

Figure 7.7: Kaplan-Meier estimator fitted to Weibull for ECO7175-T74 at 65\% UTS.

$$
\begin{aligned}
& H_{0}: \rho_{i}=1 \\
& H_{A}: \rho_{i} \neq 1
\end{aligned}
$$


Table 7.3: Statistical results of Weibull fitting of ECO7175 at $65 \%$ UTS.

\begin{tabular}{l|ccc}
\hline Weibull Distribution & ECO7175v1 & ECO7175v2 & ECO7175v3 \\
\hline N - Sample Size & 50 & 53 & 50 \\
$\alpha$ - Significance & 0.05 & 0.05 & 0.05 \\
$\lambda$ - Scale & 17345.480 & 32598.340 & 33062.880 \\
$\beta$ - Shape & 3.090 & 4.300 & 5.130 \\
SE $\lambda$ & 1870.700 & 2545.590 & 2154.2400 \\
SE $\rho$ & 0.770 & 1.030 & 1.25 \\
t-Stat $\lambda$ & 9.272 & 12.806 & 15.348 \\
t-Stat $\beta$ & 4.013 & 4.175 & 4.104 \\
t Critical & 2.011 & 2.008 & 2.011 \\
$p$-value $\lambda$ & 0.000 & 0.000 & 0.000 \\
$p$-value $\beta$ & 0.000 & 0.000 & 0.000 \\
Lower Limit $\lambda$ & 13584.186 & 27487.855 & 28731.490 \\
Upper Limit $\lambda$ & 21106.774 & 37708.825 & 37394.270 \\
Lower Limit $\beta$ & 1.542 & 2.232 & 2.617 \\
Upper Limit $\beta$ & 4.638 & 6.368 & 7.643 \\
\hline
\end{tabular}

\section{Cumulative Hazard Function}

The cumulative hazard functions of all three variants of ECO7175 were estimated and compared using the Nelson-Aalen estimator. While the cumulative hazard function is not as easy to understand as the hazard rate, its simplicity makes it a quick method to determine the hazard rate given that we understand their relation. The estimated cumulative hazard function fitted to Weibull for $75 \%$ of their tensile strength is given in Fig. 7.8. The graphs indicate ECO7175v2 and ECO7175v3 have lower accumulated hazard compared to ECO7175v1 at any given fatigue life cycle. Compared to Fig. 2.10, the curves of all three variants suggest the failures, once again, are all wear-outs. However, the Nelson-Aalen estimator performs better with a larger sample size. With a small sample size, the estimated cumulative hazard function may 
not be as well behaved, such as in the estimated cumulative hazard function at $55 \%$ of their tensile strength (Fig. 7.9).
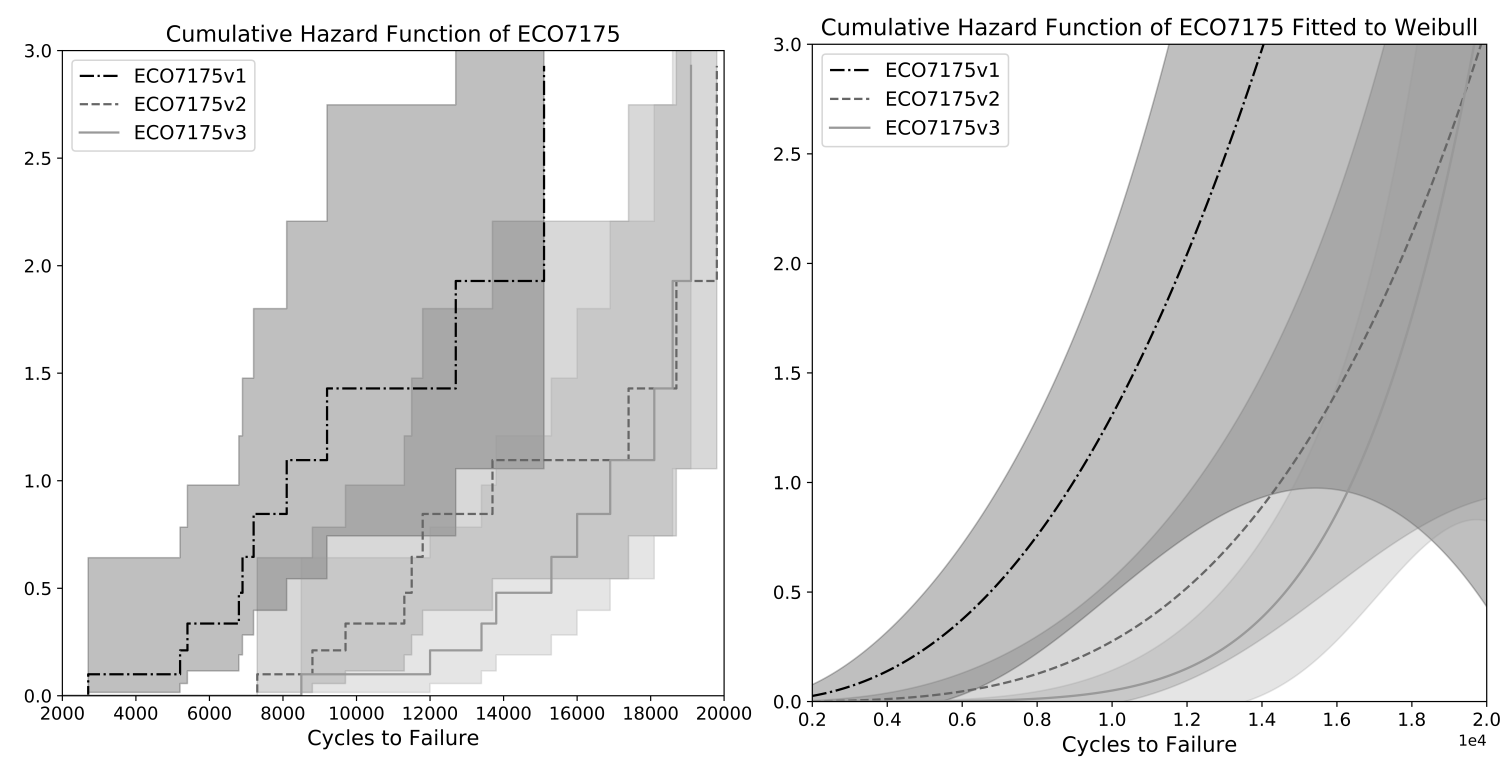

Figure 7.8: Cumulative hazard function of ECO7175 estimated with the NelsonAalen estimator at $75 \%$ UTS and then fitted to the Weibull distribution.
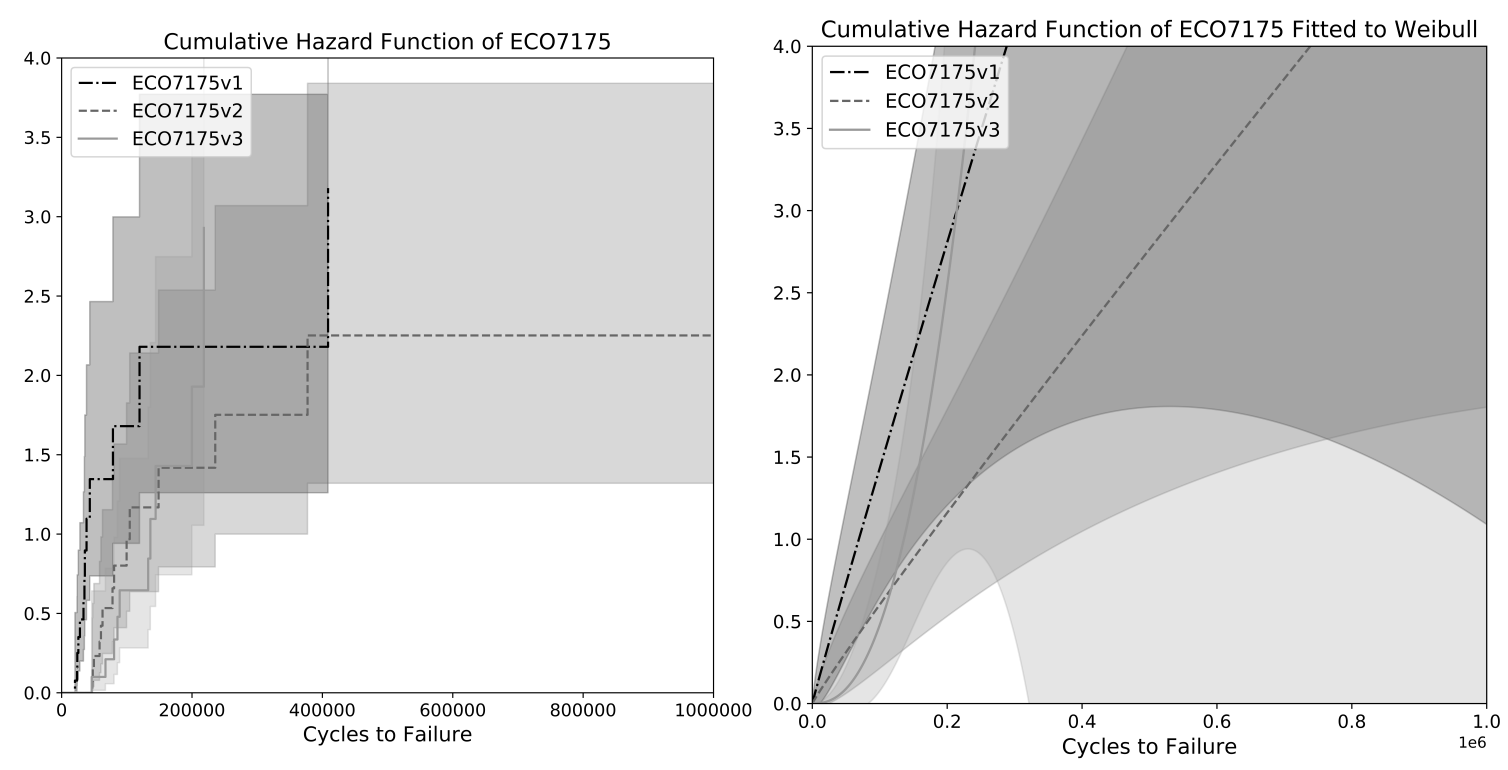

Figure 7.9: Cumulative hazard function of ECO7175 estimated with the NelsonAalen estimator at 55\% UTS and then fitted to the Weibull distribution. 


\section{Hazard Rate}

As there are limitations to the Nelson-Aalen estimator, which may cause unreliable estimates, the hazard rates of the ECO7175 were calculated directly. The graph for the calculated hazard rate of ECO7175v1 at 85\% UTS is presented in Fig. 7.10, 75\% UTS (Fig.7.11, 65\% UTS (Fig. 7.12, 55\% UTS (Fig. 7.13), and 45\% UTS (Fig. 7.14). For all stress levels, hazard rates for every variant start out low and stay relatively constant before increasing significantly toward the end. when comparing the hazard rate trends of all variants across all stress levels to the bathtub curve (Fig. 2.8), the general trends indicate all failures are wear-outs and are not caused by defects. In all cases, at any given fatigue life cycle and stress level, the hazard rates of ECO7175v2 and ECO7175v3 increase at higher fatigue life cycles compared to ECO7175v1. The results indicate the likelihood that ECO7175v2 and ECO7175v3 will fail is lower than ECO7175v1 at any given fatigue life cycle. 


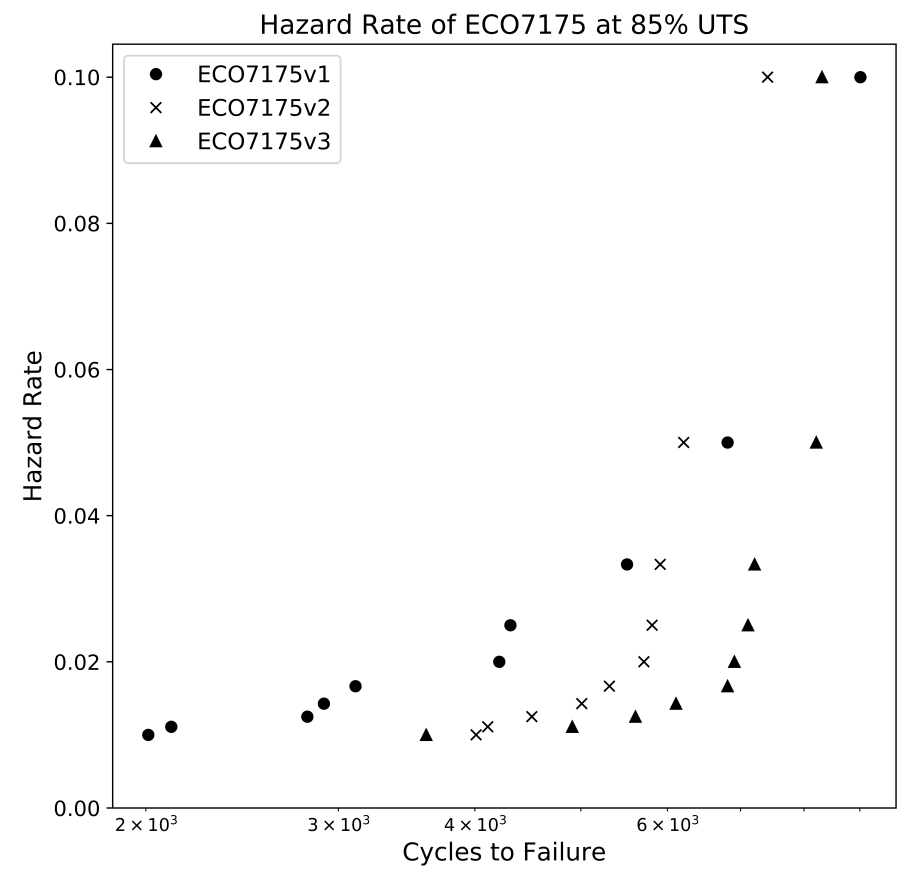

Figure 7.10: Calculated hazard rate for ECO7175 at 85\% UTS.

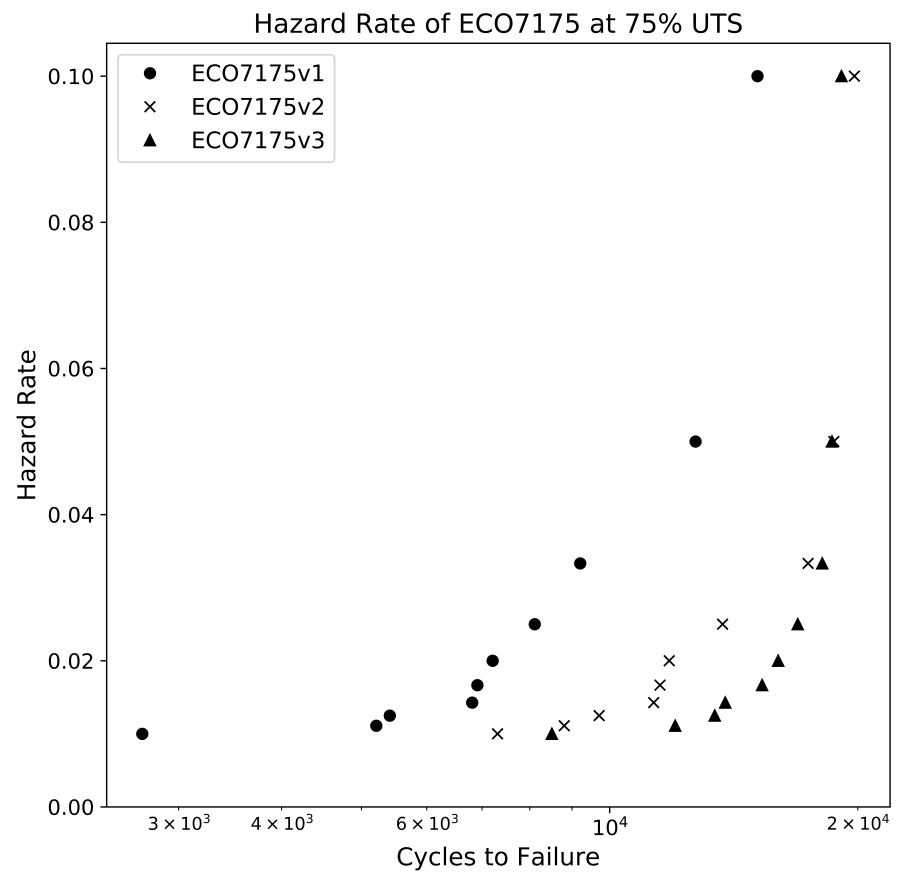

Figure 7.11: Calculated hazard rate for ECO7175 at 75\% UTS. 


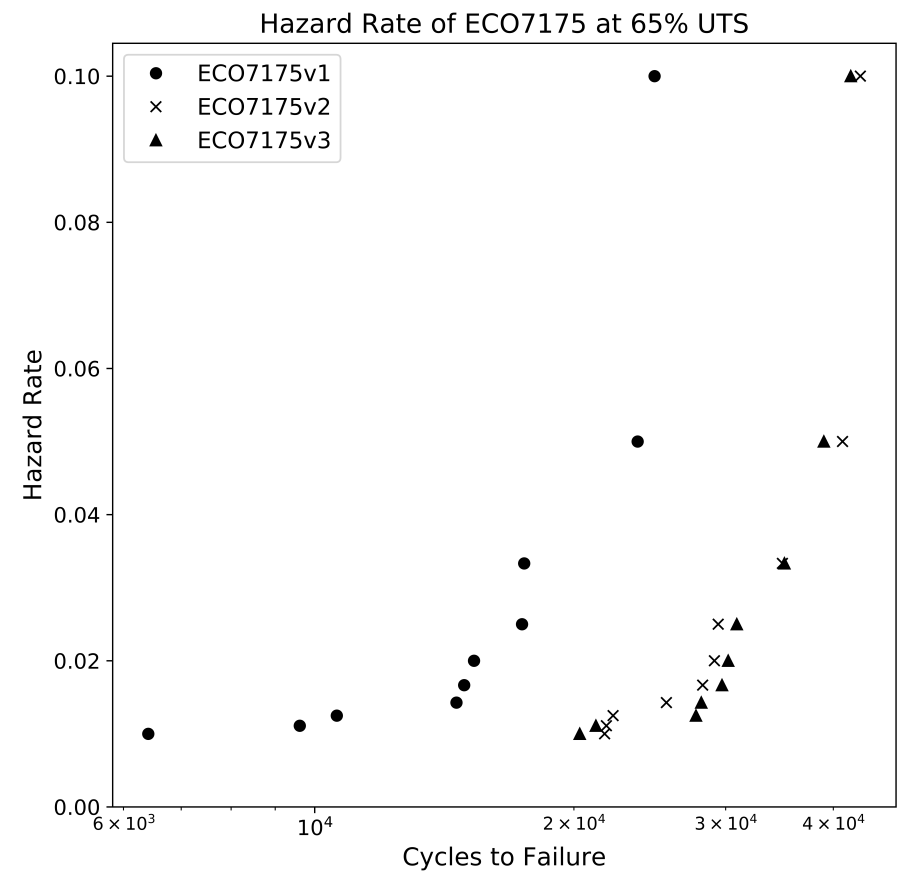

Figure 7.12: Calculated hazard rate for ECO7175 at $65 \%$ UTS.

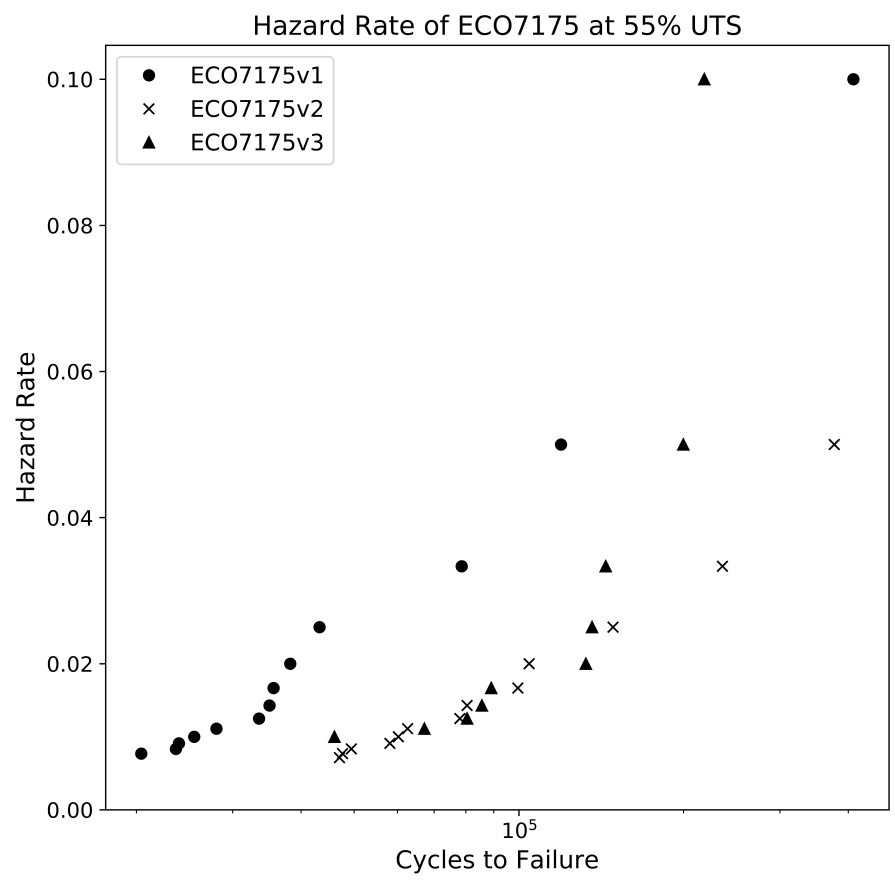

Figure 7.13: Calculated hazard rate for ECO7175 at 55\% UTS. 


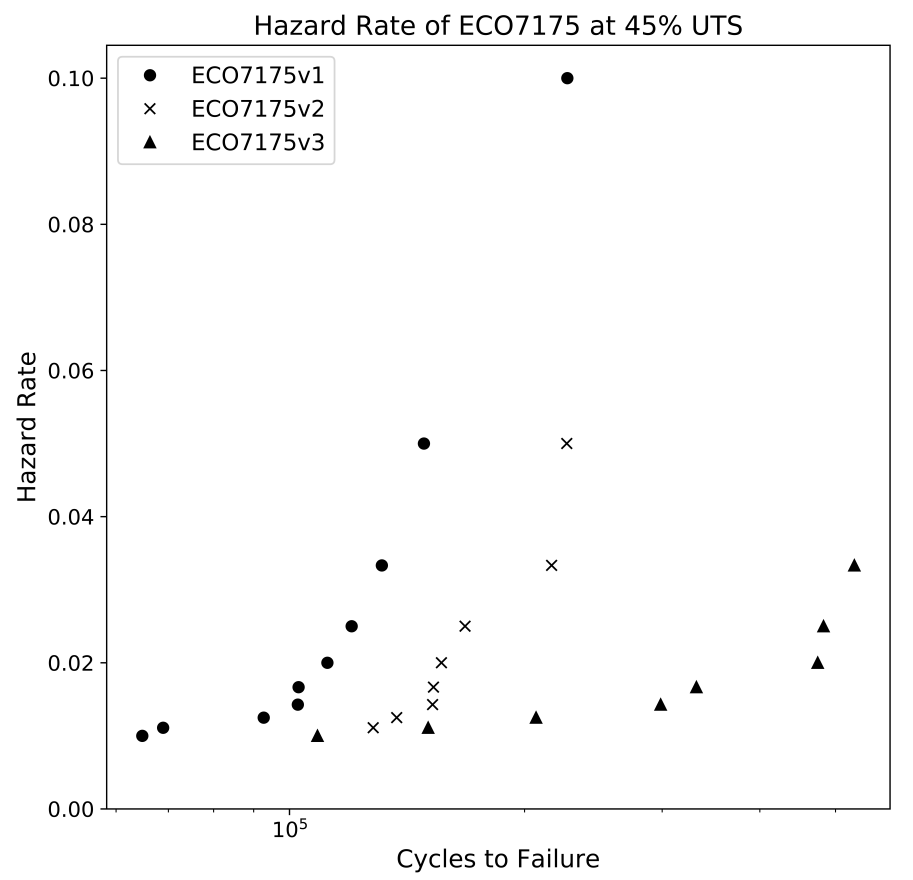

Figure 7.14: Calculated hazard rate for ECO7175 at $45 \%$ UTS.

The PDF was also calculated to determine the reliability $(R(t)=f(t) / h(t))$, which is then compared to the Kaplan-Meier estimator. The comparison is shown in Fig. 7.15. The calculated reliability and the estimated reliability through the use of the Kaplan-Meier estimator agreed. This shows the efficiency and simplicity of using the Kaplan-Meier to estimate the reliability directly. However, the hazard rates can only be estimated indirectly through the Nelon-Aalen estimator. This introduces additional errors which can increase with small sample sizes. 

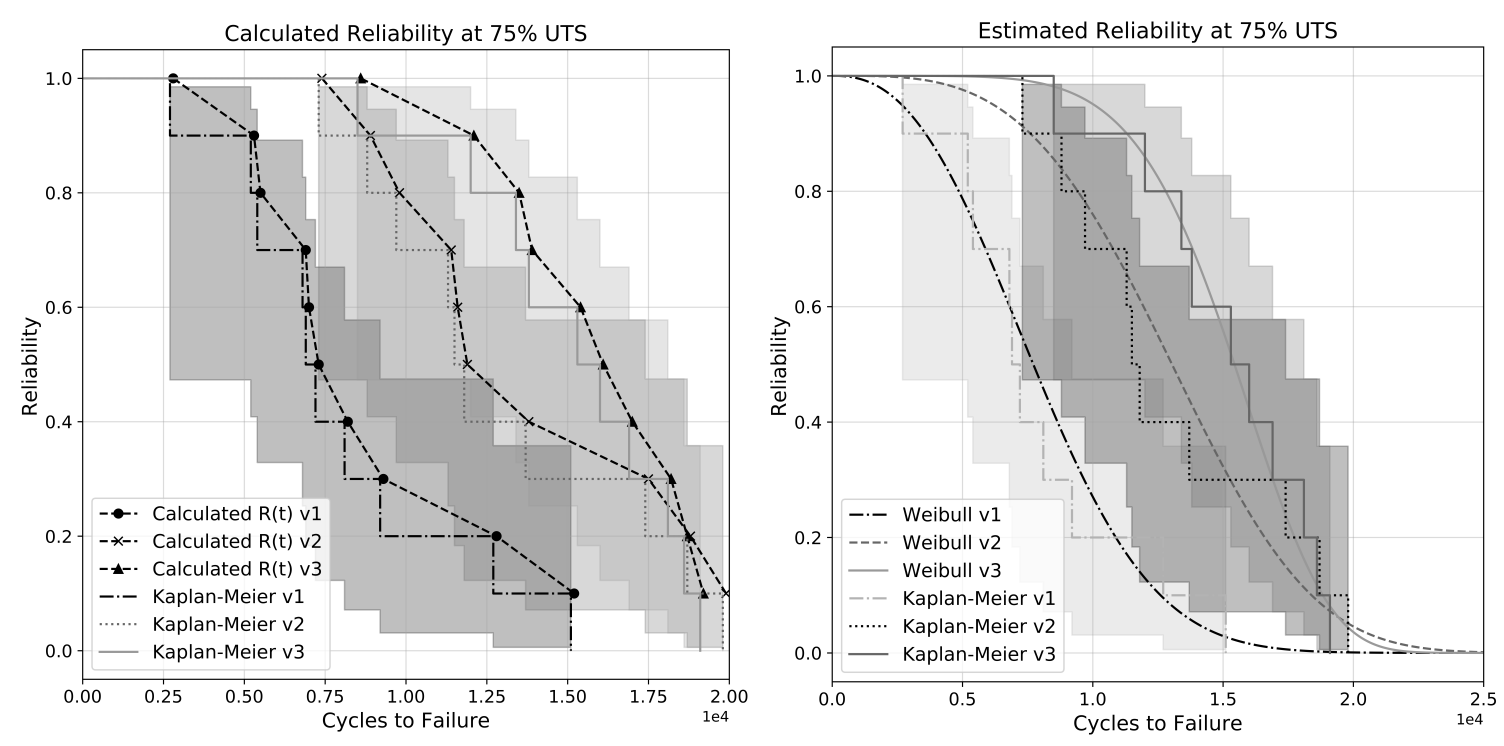

Figure 7.15: The left figure shows the calculated reliability and the estimated reliability using Kaplan-Meier. The results agree but the Kaplan-Meier is much simpler and efficient to implement. The right figure shows the estimated reliability and its Weibull fitting result.

\section{Failure Probability}

The probability of failures were also calculated for all variants at all stress levels. The graph for the failure probability at $85 \%$ UTS is presented in Fig. 7.16, 75\% UTS (Fig.7.17, 65\% UTS (Fig. 7.18, 55\% UTS (Fig. 7.19), and 45\% UTS (Fig. 7.20). In all cases, at any given fatigue life cycle and stress level, ECO7175v1 is shown to have a higher probability of failure compared to ECO7175v2 and ECO7175v3. At the same time, at any given fatigue life cycle and stress level, ECO7175v3 is shown to have a lower probability of failure compared to the other two variants. 


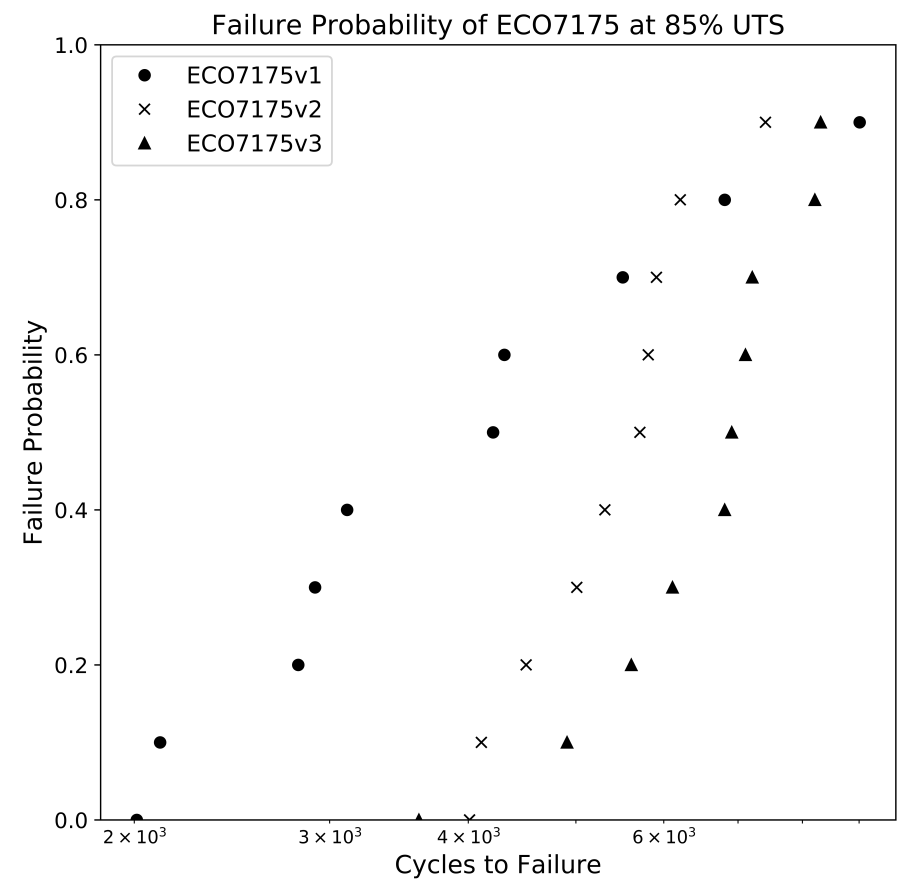

Figure 7.16: Probability of failure for ECO7175 at $85 \%$ UTS.

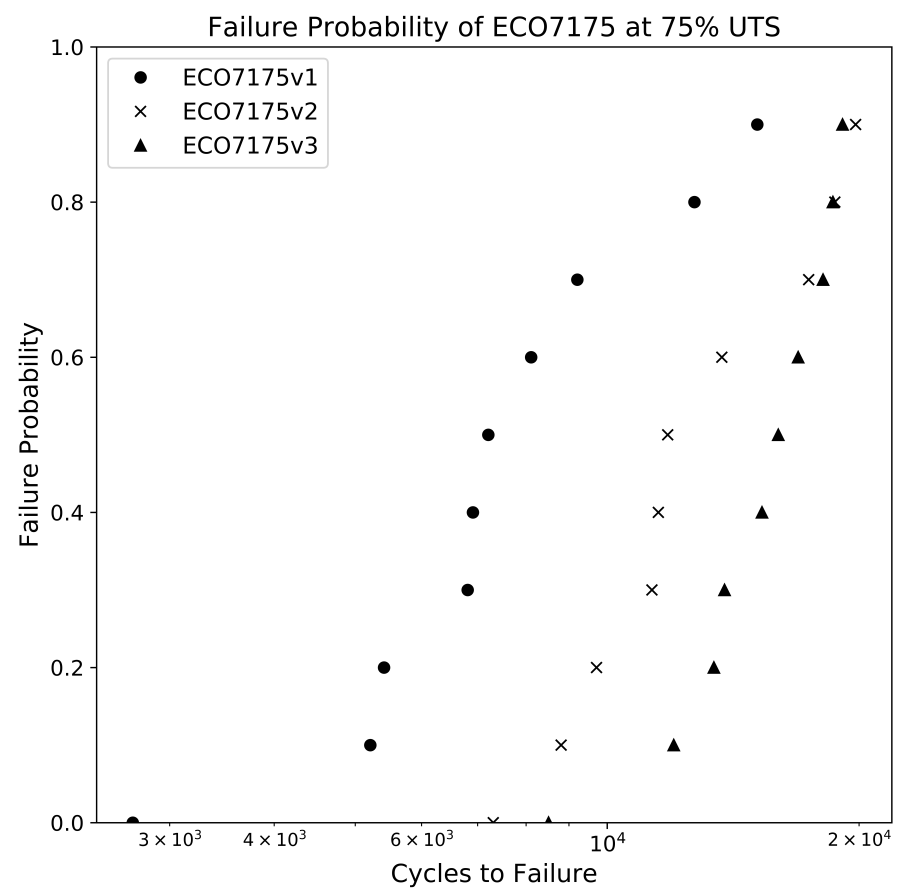

Figure 7.17: Probability of failure for ECO7175 at $75 \%$ UTS. 


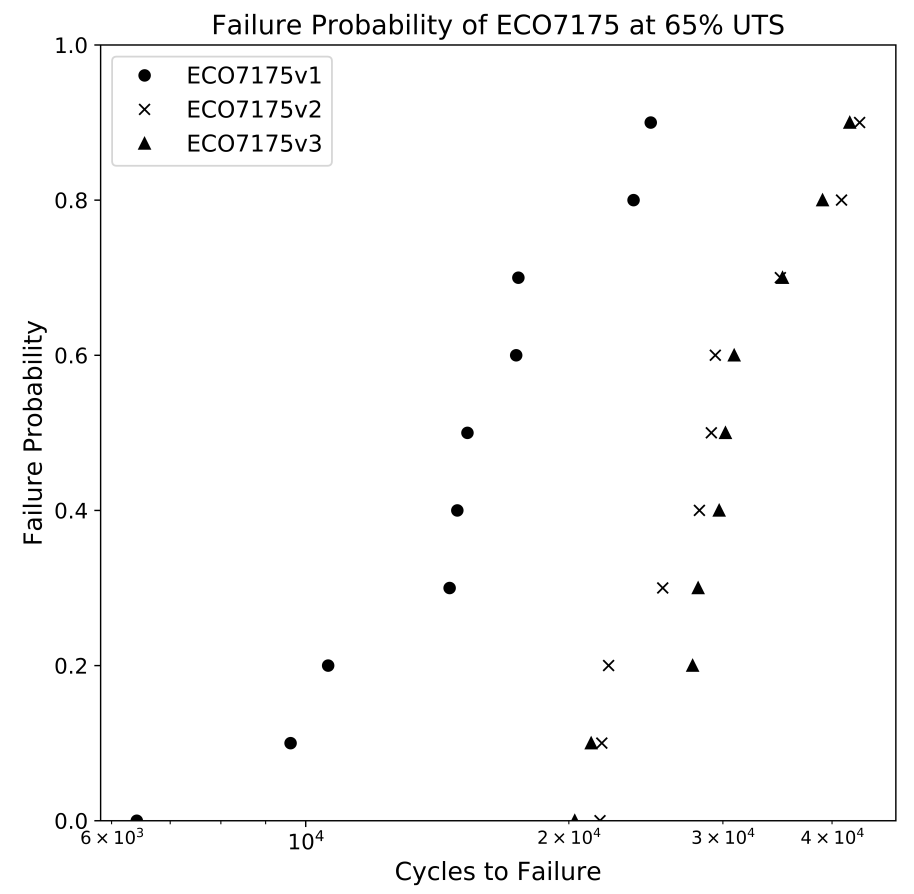

Figure 7.18: Probability of failure for ECO7175 at $65 \%$ UTS.

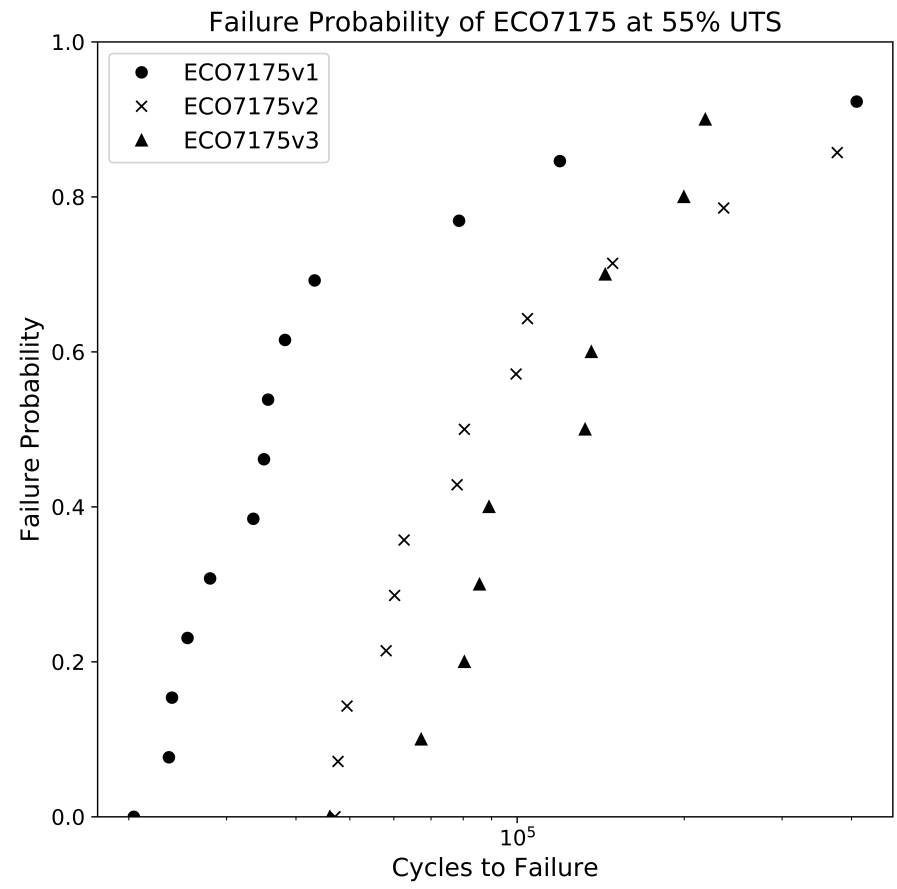

Figure 7.19: Probability of failure for ECO7175 at 55\% UTS. 


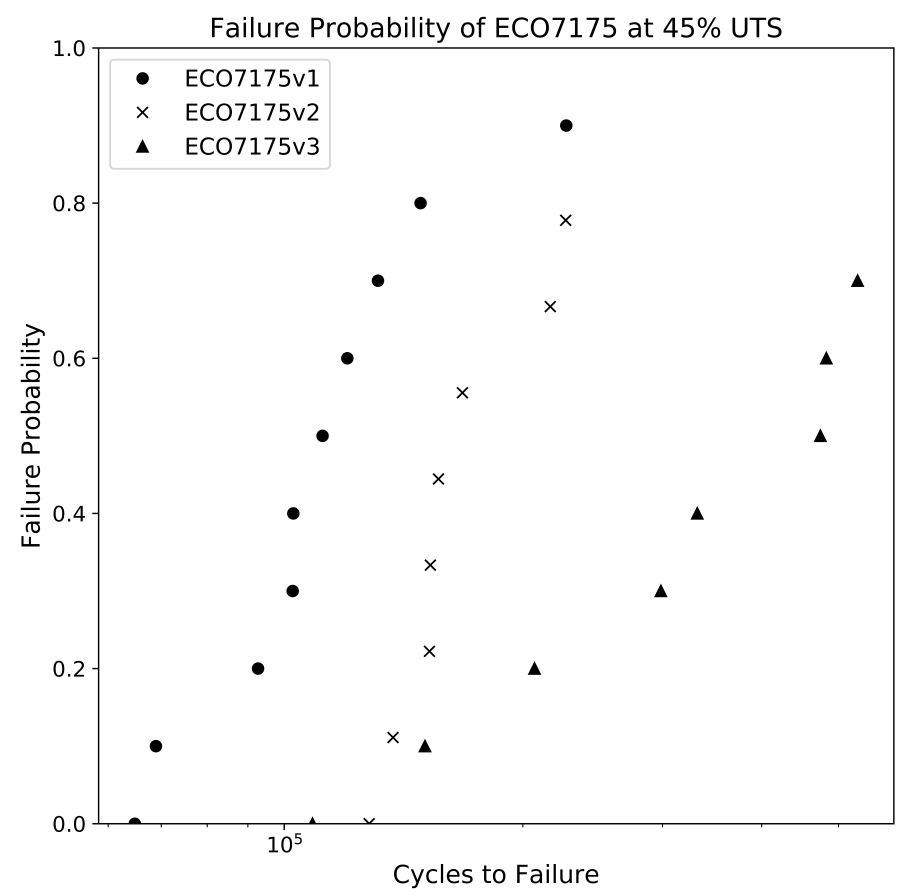

Figure 7.20: Probability of failure for ECO7175 at $45 \%$ UTS.

\section{Log-Rank Test}

The hazard rates calculated in Section 7.4 at several stress levels are shown to intersect or are not in parallel with the hazard rate curves of the other variants at a given stress level. Cases, where these occur, indicate the hazard rates are not a factor of each other, violating the proportional hazard assumption. As a result, a log-rank test is not the most appropriate test and the restricted mean survival time (RMST) may be more appropriate [45]. However, useful information can still be acquired from the log-rank tests but must be dealt with caution.

With a significance, $\alpha$, of 0.05 , the results of the log-rank tests are as followed (Table 7.4). When ECO7175v1 was compared to ECO7175v2, at all stress levels except 
at $85 \%$ of their tensile strength, the p-values are lower than $\alpha$. This indicates there are significant differences between their reliability. When ECO7175v1 was compared to ECO7175v3, once again the p-values at all stress levels except at $85 \%$ of their tensile strength shows there are significant differences between their reliability. When ECO7175v2 was compared to ECO7175v3, in all cases, the p-values indicate there are no significant differences between their reliability.

The reliability curves of all variants at any given fatigue life cycle and stress level indicate ECO7175v1 have lower reliability when compared to the other two variants. The reliability curves at any given fatigue life cycle and stress level also indicate ECO7175v3 have higher reliability than ECO7175v2. The results of the log-rank tests here show there are significant differences between ECO7175v3 and the other two variants. However, the results of the log-rank tests also show that while there are seemingly differences between the reliability for ECO7175v3 and ECO7175v2, there are not as significant. However, as the proportional hazard assumption is violated, analysis using other methods, such as RMST, may give more appropriate conclusions regarding differences between the reliability curves of each variant of ECO7175 at all stress levels.

Table 7.4: Statistical results of log-rank test for ECO7175.

\begin{tabular}{c|ccc}
\hline Log-rank Test & v1 vs v2 & v1 vs v3 & v2 vs v3 \\
\hline p-value at 85\% UTS & 0.65 & 0.21 & 0.06 \\
p-value at 75\% UTS & 0.01 & $<0.005$ & 0.69 \\
p-value at 65\% UTS & $<0.005$ & $<0.005$ & 0.99 \\
p-value at 55\% UTS & 0.03 & 0.04 & 0.87 \\
p-value at 45\% UTS & 0.02 & $<0.005$ & 0.12 \\
\hline
\end{tabular}




\subsection{Basquin's Equation}

Table 7.5 summarizes the results of curve-fitting the fatigue data to Basquin's equation (Eq. 2.7). According to Suresh, the fatigue strength coefficient, $\sigma_{F}^{\prime}$, is approximately equal to the material's fracture strength, where it is the true stress that causes the material to fail at one stress reversal, or half a stress cycle. The fatigue strength coefficient, $\sigma_{F}^{\prime}$, were determined for all three variants. Ranking of highest fatigue strength coefficient to lowest, ECO7175v3 has the highest while ECO7175v2 has the lowest. ECO7175v1 has slightly higher fatigue strength coefficient than ECO7175v2. As the fatigue strength coefficient represents the approximate materials' fracture strength, ECO7175v3 would require considerably higher stress compared to the other two variants for it to fail within one stress reversal. This is attributed by its significantly higher amounts of precipitates which act as cracks inhibitors. The fatigue strength exponent, $b$, are the same for all variants as they are basically the same material with slightly different compositions.

Table 7.5: Basquin's Constants of ECO7175.

\begin{tabular}{ccc}
\hline Variant & $\sigma_{F}^{\prime}(\mathrm{MPa})$ & $b$ \\
\hline ECO7175v1-T74 & 1557.6 & -0.145 \\
ECO7175v2-T74 & 1547.1 & -0.145 \\
ECO7175v3-T74 & 1589.7 & -0.145 \\
\hline
\end{tabular}




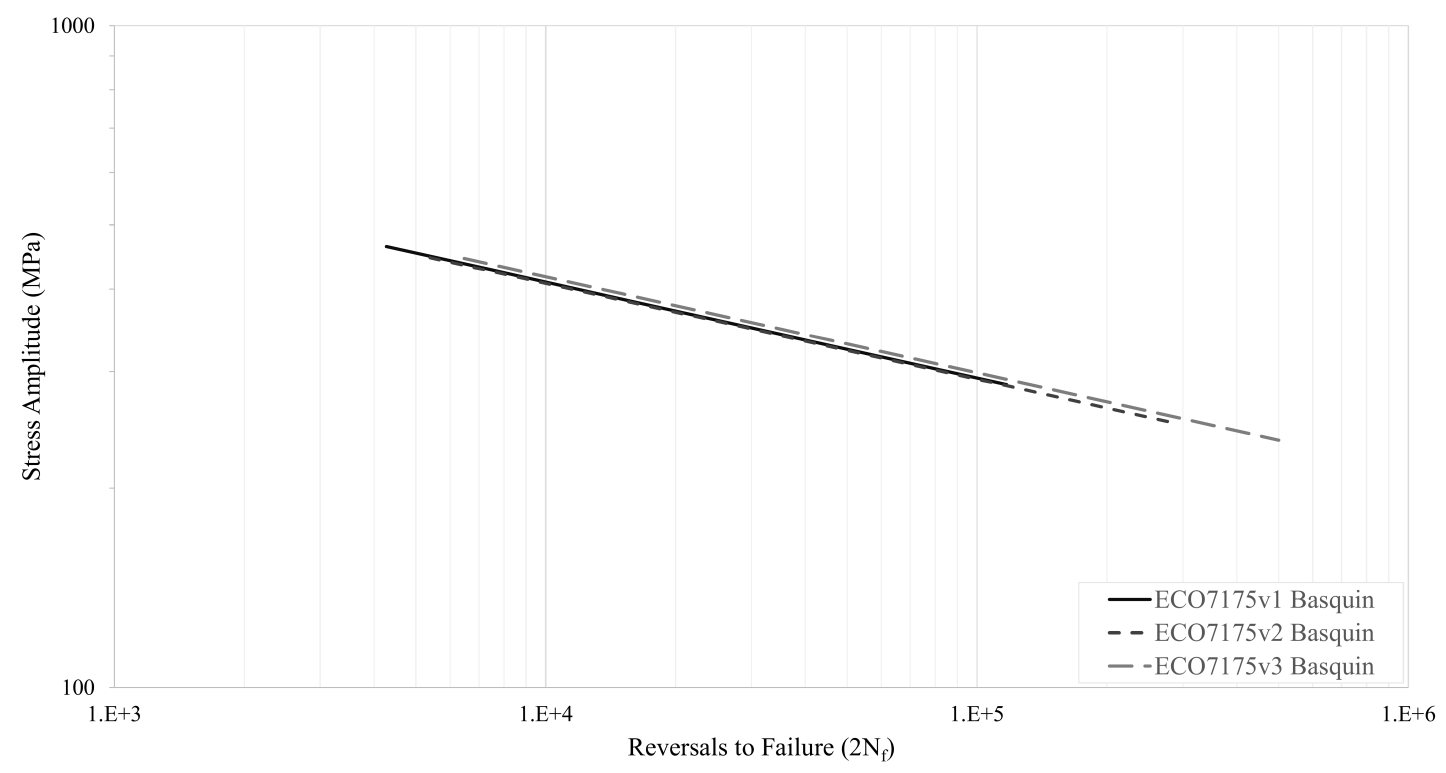

Figure 7.21: Plot of Basquin's equations for all three variants of ECO7175. ECO7175v3 is shown to have a considerably higher fatigue strength coefficient compared to ECO7175v1-T74 and ECO7175v2-T74. 


\section{Chapter 8}

\section{Conclusion}

In this dissertation, the fatigue characteristics of three newly developed experimental compositions for aluminum 7175 that uses magnesium-calcium alloy instead of pure magnesium, with improved mechanical strength, are studied.

Specimens of each variant were fabricated and subjected to fatigue life tests, fatigue life data analysis, and observation of their fracture characteristics through optical microscopy and scanning electron microscopy (SEM), and metallography to study their grains and surface characteristics.

Fatigue life tests show all three variants have a fatigue strength that is approaching approximately $200 \mathrm{MPa}$. ECO7175v3 is shown to have the highest fatigue strength of approximately $220 \mathrm{MPa}$ at $5 \times 10^{7}$ cycles, approximately $40 \%$ of its tensile strength of $550 \mathrm{MPa}$. This is shown by its considerably higher fatigue strength coefficient determined by Basquin's equation compared to the other two variants. ECO7175v1 is shown to generally have large scatter in its fatigue life at higher stress levels $(65 \%$ or higher of their tensile strength) (Fig. 4.2) with coefficient of variations (Table 4.4) typically twice or more to those of ECO7175v2 and ECO7175v3 (Table 6.3 and 5.3).

Fractography analysis has shown that ECO7175v3 has unique fracture characteristics for specimens that are subjected to $67 \%$ or lower of their tensile strength (Fig. 5.5). Its fracture surfaces are softly defined with propagation bands that are hard 
to discern and the fracture initiation points are hard to locate with the unaided eye. ECO7175v3's unique fracture characteristics are attributed to its significantly larger grain size of approximately $78 \mu m$ (Fig. 5.13).

The results of the SEM analysis show that irrespective of the stress levels, ECO7175v1 and ECO7175v3 all have crack initiation points at the surface with no inclusions to act as stress concentrators. The lack of inclusions is supported by the reliability analysis which shows the hazard rates for all variants remains relatively constant the majority of the time before increasing towards the end. These trends for all variants indicate failures are due to wear-outs instead of defects, which were not seen. Reliability analysis also shows that at any given fatigue life cycle and stress level, ECO7175v3 has a lower probability of failure when compared to ECO7175v1 and ECO7175v2. On the other hand, at any given fatigue life cycle and stress level, ECO7175v1 is shown to have a higher probability of failure when compared to ECO7175v2 and ECO7175v3.

Metallography analysis has shown that ECO7175v3 has considerably more precipitates with a volume fraction of approximately $2.5 \%$ (Fig. 5.19) compared to $1.6 \%$ for ECO7175v1 (Fig. 5.20) and 0.48\% for ECO7175v2 (Fig. 6.9). The larger quantity of precipitates increases ECO7175v3's fatigue strength as the crack must change direction every time a precipitate is encountered. 


\section{Bibliography}

[1] Aluminum Association. International alloy designations and chemical composition limits for wrought aluminum and wrought aluminum alloys. Arlington, VA: Aluminum Association, 2015.

[2] Joseph R. Davis. ASM specialty handbook: Aluminum and aluminum alloys. Materials Park, OH: ASM International, 1993.

[3] Seong Taek Lim, Il Sang Eun, and Soo Woo Nam. "Control of equilibrium phases (M,T,S) in the modified aluminum alloy 7175". In: Materials Transactions. Vol. 44. 1. 2003, pp. 181-187. DOI: doi:10.2320/matertrans.44.181.

[4] Seon Ho Kim et al. "Microstructure and Mechanical Properties of Eco-2024-T3 aluminum alloy". In: Advanced Materials Research (Volumes 602-604 (2012), pp. 623-626. DOI: doi:10.4028/www.scientific.net/AMR.602-604.623.

[5] T.H. Nam et al. "Corrosion resistance of extruded Mg-3Al-1Zn alloy manufactured by adding $\mathrm{CaO}$ for the replacement of the protective gases". In: Materials and Corrosion 577-581. Vol. 31. 6. 2014. DOI: doi:10.1002/maco.201206849.

[6] Shae K. Kim et al. "Green manufacturing for magnesium alloys". In: 67th World Foundry Congress (wfc06): Casting the Future. West Midlands, UK: Institute of Cast Metals Engineers, 2006, pp. 1-34. 
[7] Shae K. Kim and Jin-Kyu Lee. "Effect of $\mathrm{CaO}$ addition on the ignition resistance of Mg-Al alloys". In: Materials Transactions 52.7 (2011), pp. 1483-1488. DoI: doi: 10.2320/matertrans.M2010397.

[8] Jae-Kyoo Lim et al. "Fatigue crack growth behavior for 7000 series high strength $\mathrm{Al}$ alloys on strengthening heat treatment". In: Fatigue '96: Sixth International Fatigue Congress. Ed. by G. Lütjering H. Nowack. Berlin, Germany: Pergamon, 1996, pp. 947-952.

[9] Aline Chemin et al. "Influence of $\mathrm{Al} 7 \mathrm{Cu} 2 \mathrm{Fe}$ intermetallic particles on the localized corrosion of high strength aluminum alloys". In: Materials $\&$ Design (2014), pp. 118-123. DOI: doi:10.1016/j.matdes.2013.07.003.

[10] Vladimir V. Bolotin. Mechanics of Fatigue. Boca Raton, Florida: CRC Press, 1999.

[11] M. Fuentes et al. Fracture Mechanics: Applications and Challenges. Vol. 26. European Structural Integrity Society. Spain: Elsevier, 2000.

[12] Theodore Nicholas. High Cycle Fatigue: A Mechanics of Materials Perspective. Oxford, UK: Elsevier Science, 2006.

[13] Versailles train crash in 1842. 1842. URL: https://commons.wikimedia.org/ wiki/File:Meudon_1842.jpg. 
[14] Sven Glagow. Das ICE-Unglück von Eschede. 2018. URL: https://www.ndr.de/ kultur/geschichte/Das-ICE-Unglueck-von-Eschede, eschededossier2. html.

[15] S. Suresh. Fatigue of Materials, 2nd Edition. Cambridge, UK, 1998.

[16] Claude Bathias and Paul C. Paris. Gigacycle Fatigue in Mechanical Practice. Cambridge, UK, 1998.

[17] Agnieszka Betzwar Kotas et al. "Fatigue testing and properties of hardmetals in the gigacycle range". In: International Journal of Refractory Metals and Hard Materials 62 (2017). Hardmetals - raw materials, technologies and applications, pp. 183-191. ISSN: 0263-4368. DOI: https : //doi .org/10 .1016/j . ijrmhm . 2016.07 .004. URL: http : //www. sciencedirect.com/science/article/ pii/S026343681630172X.

[18] L.F. Coffin Jr. "A Study of the Effects of Cyclic Thermal Stresses on a Ductile Metal". In: Transactions of the ASME (1954), pp. 931-950.

[19] S.S. Manson. Behavior of materials under conditions of thermal stress. Cleveland, 1954.

[20] H. Xu, T. Lee, and C. Kim. "Fatigue properties of lead-free solder joints in electronic packaging assembly investigated by isothermal cyclic shear fatigue". In: 2014 IEEE 64th Electronic Components and Technology Conference (ECTC). May 2014, pp. 133-138. DOI: 10.1109/ECTC.2014.6897278. 
[21] O.H. Basquin. The exponential law of endurance tests. 1910.

[22] J.D. Morrow. Fatigue Design Handbook-Advances in Engineering. Vol. 4. Warrendale, PA: Society of Automotive Engineers, 1968, pp. 21-29.

[23] F. M. Safie and R. P. Fuller. "NASA applications and lessons learned in reliability engineering". In: 2012 Proceedings Annual Reliability and Maintainability Symposium. Jan. 2012, pp. 1-5. DOI: 10.1109/RAMS.2012.6175423.

[24] Elsayed A. Elsayed. Reliability Engineering. 2nd ed. Wiley, 2012.

[25] G. A. Klutke, P. C. Kiessler, and M. A. Wortman. "A critical look at the bathtub curve". In: IEEE Transactions on Reliability 52.1 (Mar. 2003), pp. 125-129. ISSN: 0018-9529. DOI: 10.1109/TR.2002.804492.

[26] G. A. Bohoris. "Comparison of the cumulative-hazard and Kaplan-Meier estimators of the survivor function". In: IEEE Transactions on Reliability 43.2 (June 1994), pp. 230-232. ISSN: 0018-9529. DOI: 10.1109/24.294997.

[27] E. L. Kaplan and Paul Meier. "Nonparametric estimation from incomplete observations". In: Journal of the American Statistical Association 53.282 (1958), pp. 457-481. DOI: doi:10.2307/2281868.

[28] Wayne Nelson. "Theory and Applications of Hazard Plotting for Censored Failure Data". In: Technometrics 14.4 (1972), pp. 945-966. DOI: doi : 10.2307/ 1267144. 
[29] Arthur V. Peterson Jr. "Expressing the Kaplan-Meier Estimator as a Function of Empirical Subsurvival Functions". In: Journal of the American Statistical Association 72.360 (1977), pp. 854-858. DOI: doi:10.2307/2286474.

[30] N. Mantel. "Evaluation of survival data and two new rank order statistics arising in its consideration". In: Cancer Chemotherapy Reports 50.3 (Mar. 1966). [PubMed:5910392], pp. 163-170.

[31] Richard Peto and Julian Peto. "Asymptotically Efficient Rank Invariant Test Procedures". In: Journal of the Royal Statistical Society. Series A (General) 135.2 (1972), pp. 185-207. ISSN: 00359238. URL: http : / / www . jstor . org / stable/2344317.

[32] David Harrington. "Linear Rank Tests in Survival Analysis". In: Encyclopedia of Biostatistics. American Cancer Society, 2005. ISBN: 9780470011812. DOI: 10 . 1002/0470011815 . b2a11047. eprint: https : / / onlinelibrary . wiley . com/ doi /pdf / 10 . 1002/0470011815 . b2a11047. URL: https : / / onlinelibrary . wiley.com/doi/abs/10.1002/0470011815.b2a11047.

[33] Charles Lipson and Narendra J. Sheth. Statistical design and analysis of engineering experiments. New York: McGraw-Hill, 1973.

[34] Battelle Memorial Institute. Metallic Materials Properties Development and Standardization (MMPDS). Columbus, Ohio: Battelle Memorial Institute, 2016. 
[35] American Society for Metals. Metals Handbook 9th Edition. Ohio, Metals Park: American Society for Metals, 1985.

[36] Cameron Davidson-Pilon. lifelines. https : / / doi . org / 10 . 5281/ zenodo . 2577595. 2019.

[37] Gilbert J. Kaufman. Introduction to aluminum alloys and tempers. Materials Park, OH: ASM International, 2000.

[38] Chinh Vu et al. "Fatigue Characteristic Analysis of New ECO7175v1 Extruded Aluminum Alloy". In: Journal of Aerospace Engineering 32.1 (2019). DOI: doi : 10.1061/(ASCE) AS . 1943-5525.0000958.

[39] H. Mayer et al. "Very high cycle fatigue properties of bainitic high carbonchromium steel". In: 31.2 (2009), pp. 242-249. DOI: doi:10.1016/j . ijfatigue. 2014.05 .001$.

[40] Y. Ochi et al. "High-cycle rotating bending fatigue property in very long-life regime of high-strength steels". In: Fatigue $\&$ Fracture of Engineering Materials E Structures 25 (2002), pp. 8-9. DOI: doi : 10.1046/j.1460-2695.2002.00575.

[41] K. Shiozawa, L. Lu, and S. Ishihara. "S-N curve characteristics and subsurface crack initiation behaviour in ultra-long life fatigue of a high carbon-chromium bearing steel". In: Fatigue $\&$ Fracture of Engineering Materials 8 Structures 24.12 (2001), pp. 781-790. DOI: doi:10.1046/j.1460-2695.2001.00459. 
[42] Q.Y. Wang, N. Kawagoishi, and Q. Chen. "Fatigue and fracture behaviour of structural Al-alloys up to very long life regimes". In: International Journal of Fatigue 28.11 (2006), pp. 1572-1576. DOI: doi:10.1016/j.ijfatigue. 2005. 09.017.

[43] R. Ahmad. "The Effect of Chromium Addition on Fluidity, Microstructure and Mechanical Properties of Aluminium LM6 Cast Alloy". In: International Journal of Material Science and Research 1.1 (2018), pp. 32-35. DOI: doi : 10 . 18689/ijmsr-1000105.

[44] Kenneth G. Budinski and Michael K. Budinski. Engineering Materials: Properties and Selection. Ohio, Metals Park: Pearson, 2009.

[45] Lu Tian et al. "Efficiency of two sample tests via the restricted mean survival time for analyzing event time observations". In: Biometrics 74.2 (2018), pp. 694-702. DOI: 10.1111/biom. 12770. eprint: https ://onlinelibrary . wiley.com/doi/pdf/10.1111/biom.12770. URL: https://onlinelibrary . wiley.com/doi/abs/10.1111/biom.12770. 